\title{
Critical Science Plan for the Daniel K. Inouye Solar Telescope (DKIST)
}

Mark P. Rast ${ }^{1,2}(\mathbb{D}) \cdot$ Nazaret Bello González $^{3}$ - Luis Bellot Rubio ${ }^{4}(\mathrm{D}){\text { Wenda } \text { Cao }^{5}(\mathrm{D})}^{-}$ Gianna Cauzzi $^{6}$ (D) Edward DeLuca ${ }^{7}$ (D) Bart De Pontieu $^{8,9}$ (D) Lyndsay Fletcher $^{10,9}$ (D) - Sarah E. Gibson ${ }^{11}$ (D) Philip G. Judge ${ }^{11}($ D $\cdot$ Yukio Katsukawa ${ }^{12,13}$ (D) Maria D. Kazachenko ${ }^{1,6}$ (D) Elena Khomenko ${ }^{14}$ (D) Enrico Landi ${ }^{15}(\mathbb{D}) \cdot$ Valentín Martínez Pillet $^{6}\left(\mathbb{D} \cdot\right.$ Gordon J.D. Petrie $^{6}\left(\mathbb{D} \cdot\right.$ Jiong Qiu $^{16}$. Laurel A. Rachmeler ${ }^{17}$ (D) Matthias Rempel ${ }^{11}$ (D) - Wolfgang Schmidt ${ }^{3}$.

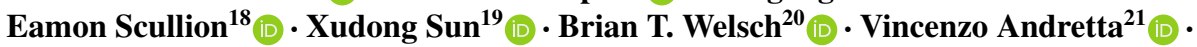
Patrick Antolin $^{18}$ (D) Thomas R. Ayres ${ }^{22}$ D - K.S. Balasubramaniam ${ }^{23}$. Istvan Ballai $^{24}$ - Thomas E. Berger ${ }^{2}$ Stephen J. Bradshaw ${ }^{25}$ (D)

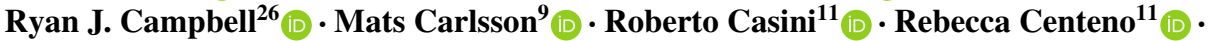
Steven R. Cranmer ${ }^{1,2}(D)$ Serena Criscuoli ${ }^{6}(D)$ Craig DeForest $^{27}(D) \cdot$ Yuanyong Deng ${ }^{28} \cdot$ Robertus Erdélyi $^{24}(D)$ Viktor Fedun $^{29} \cdot$ Catherine E. Fischer $^{3}$ (D) Sergio J. González Manrique ${ }^{30}$ (D) Michael Hahn ${ }^{31}$ (D) - Louise Harra ${ }^{32}$ (D) Vasco M.J. Henriques $^{9}\left(D \cdot\right.$ Neal E. Hurlburt $^{8}(D) \cdot$ Sarah Jaeggli $^{6}(D)$ Shahin Jafarzadeh ${ }^{9}$ - Rekha Jain ${ }^{24}$ - Stuart M. Jefferies ${ }^{33}$ • Peter H. Keys ${ }^{26}$. Adam F. Kowalski ${ }^{1,6}$ - Christoph Kuckein ${ }^{34}$ - Jeffrey R. Kuhn ${ }^{19}$ (D)

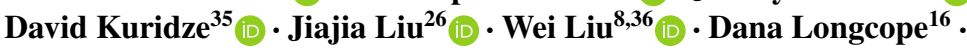
Mihalis Mathioudakis $^{26}$ (D) R.T. James McAteer ${ }^{37}$ (D) Scott W. McIntosh' ${ }^{11}$ (D) David E. McKenzie ${ }^{38}$ - Mari Paz Miralles ${ }^{7} \cdot$ Richard J. Morton $^{18}$. Karin Muglach $^{39,40}(\mathrm{D}) \cdot$ Chris J. Nelson ${ }^{26}$ D * Navdeep K. Panesar ${ }^{8,36}$ • Susanna Parenti ${ }^{41}(D)$. Clare E. Parnell ${ }^{42}(D) \cdot$ Bala Poduval $^{43}(D) \cdot$ Kevin P. Reardon $^{6}$. Jeffrey W. Reep $^{44}$ (D) Thomas A. Schad ${ }^{6}$ (D) Donald Schmit ${ }^{2}$ (D) Rahul Sharma $^{45,18}$ (D) Hector Socas-Navarro $^{14}(\mathrm{D}) \cdot$ Abhishek K. Srivastava $^{46}$ - Alphonse C. Sterling ${ }^{38}$ (D) Yoshinori Suematsu $^{13}(\mathrm{D}) \cdot$ Lucas A. Tarr $^{6}$ (D) Sanjiv Tiwari ${ }^{8,36}{ }^{(D)}$. Alexandra Tritschler ${ }^{6}$ D Gary Verth $^{24}$ - Angelos Vourlidas ${ }^{47}$ (D) Haimin Wang ${ }^{5}$ (D) Yi-Ming Wang ${ }^{44}$ - NSO, DKIST project, and DKIST instrument scientists ${ }^{48}$. the DKIST Science Working Group ${ }^{48}$ - the DKIST Critical Science Plan Community ${ }^{48}$

Received: 13 August 2020 / Accepted: 16 February 2021 / Published online: 19 April 2021

(C) The Author(s) 2021

This article belongs to the Topical Collection:

The Daniel K. Inouye Solar Telescope (DKIST)

Guest Editors: Thomas R. Rimmele, Philip R. Goode, Michael Knölker, Jeffrey R. Kuhn, and Robert Rosner

M.P. Rast

mark.rast@lasp.colorado.edu

1 Department of Astrophysical and Planetary Sciences, University of Colorado, Boulder, CO 80309, USA 


\begin{abstract}
The National Science Foundation's Daniel K. Inouye Solar Telescope (DKIST) will revolutionize our ability to measure, understand, and model the basic physical processes that control the structure and dynamics of the Sun and its atmosphere. The first-light DKIST images, released publicly on 29 January 2020, only hint at the extraordinary capabilities that will accompany full commissioning of the five facility instruments. With this Critical Science Plan (CSP) we attempt to anticipate some of what those capabilities will enable, providing a snapshot of some of the scientific pursuits that the DKIST hopes to engage as start-of-operations nears. The work builds on the combined contributions of the DKIST Science Working Group (SWG) and CSP Community members, who generously shared their experiences, plans, knowledge, and dreams. Discussion is primarily focused on those issues to which DKIST will uniquely contribute.
\end{abstract}

Keywords Solar photosphere $\cdot$ Chromosphere $\cdot$ Corona

\title{
1. Introduction
}

The primary goal of the Daniel K. Inouye Solar Telescope (DKIST) is to address longstanding problems in solar physics, such as the operation of the solar dynamo and the heating and acceleration of the solar chromospheric and coronal plasma, but its scientific impact will extend well beyond the Sun. DKIST data will contribute to our understanding of fundamental physical processes, such as the generation and annihilation of magnetic field in plasmas of

2 Laboratory for Atmospheric and Space Physics, University of Colorado, Boulder, CO 80303, USA

3 Leibniz-Institut für Sonnenphysik (KIS), 79104 Freiburg, Germany

4 Instituto de Astrofísica de Andalucía (CSIC), 18080 Granada, Spain

5 Big Bear Solar Observatory, New Jersey Institute of Technology, Big Bear City, CA 92314, USA

6 National Solar Observatory, Boulder, CO 80303, USA

7 Harvard-Smithsonian Center for Astrophysics, Cambridge, MA 02138, USA

8 Lockheed Martin Solar \& Astrophysics Laboratory, Palo Alto, CA 94304, USA

9 Institute of Theoretical Astrophysics, Rosseland Centre for Solar Physics, University of Oslo, 0315 Oslo, Norway

10 School of Physics and Astronomy, University of Glasgow, Glasgow, G12 8QQ, UK

11 High Altitude Observatory, National Center for Atmospheric Research, Boulder, CO 80307, USA

12 Department of Astronomical Science, School of Physical Sciences, The Graduate University for Advanced Studies, SOKENDAI, Tokyo 181-8588, Japan

13 National Astronomical Observatory of Japan, National Institutes of Natural Science, Tokyo 181-8588, Japan

14 Instituto de Astrofísica de Canarias, 38205 La Laguna, Tenerife, Spain

15 Department of Climate and Space Sciences and Engineering, University of Michigan, Ann Arbor, MI 48109, USA

16 Department of Physics, Montana State University, Bozeman, MT 59717, USA

17 NOAA National Centers for Environmental Information, Boulder, CO 80305, USA

18 Department of Mathematics, Physics and Electrical Engineering, Northumbria University, Newcastle upon Tyne, NE1 8ST, UK 
very high electrical conductivity, the role of turbulence under extreme conditions not achievable in terrestrial laboratories, and the quantum mechanical underpinnings of polarization spectroscopy essential to the interpretation of a broad range of astrophysical observations. The anticipated high spatial and temporal resolution high-precision spectropolarimetric observations of the continuously reorganizing and reconfiguring solar magnetic field will allow detailed study of the underlying impulsive energy release and particle-acceleration mechanisms responsible for the formation of particle beams and plasma ejecta. These processes are ubiquitous in astrophysics, critical to the stability of laboratory plasmas, and directly impact our ability to robustly extend human technology into the Earth's space environment.

With a post-focus suite of five instruments (see de Wijn et al., 2021; Fehlmann et al., 2021; Jaeggli et al., 2021; von der Lühe et al., 2021; Wöger et al., 2021), the DKIST's novel capabilities come with extreme flexibility and consequent complexity. Significant effort is required to understand how to best leverage that flexibility to achieve the rich scientific goals uniquely accessible by DKIST soon after the start of operations. The strategy of the National Solar Observatory (NSO) has been to actively engage a large cross-section of the US and international solar and space physics community in defining these goals and how to achieve them. This was done, not only to expand the range of science to be pursued, but to help ensure via early assessment that the anticipated critical science can indeed be addressed using the DKIST telescope and the anticipated post-focus instrument suite.

At the heart of the DKIST Critical Science Plan, described here, are scientific goals formulated by the DKIST Science Working Group after consideration of the Science Use Cases contributed by the community via an Atlassian ${ }^{\circledR}$ Jira $^{\circledR}$ development interface. Science Use Case development was partially facilitated by a series of Critical Science Plan Workshops hosted jointly by the NSO and community partners (www.nso.edu/telescopes/

19 Institute for Astronomy, University of Hawaii, Pukalani, HI 96768, USA

20

INAF - Osservatorio Astronomico di Capodimonte, 80131 Naples, Italy

Center for Astrophysics and Space Astronomy, University of Colorado, Boulder, CO 80309, USA

AFRL Battlespace Environment Division, Albuquerque, NM 87117, USA

School of Mathematics and Statistics, The University of Sheffield, Sheffield, S3 7RH, UK

Department of Physics and Astronomy, Rice University, Houston, TX 77005, USA

Astrophysics Research Centre, School of Mathematics and Physics, Queen's University, Belfast, BT7 1NN, UK

Southwest Research Institute, Boulder, CO 80302, USA

Key Laboratory of Solar Activity, National Astronomical Observatories, Chinese Academy of Sciences, Beijing 100012, People's Republic of China

Department of Automatic Control and Systems Engineering, The University of Sheffield, Sheffield, S1 3JD, UK

Astronomical Institute of the Slovak Academy of Sciences, 05960 Tatranská Lomnica, Slovak Republic

PMOD/WRC, 7260 Davos Dorf, Switzerland

Department of Physics and Astronomy, Georgia State University, Atlanta, GA 30303, USA

Leibniz-Institut für Astrophysik, 14482 Potsdam, Germany

Department of Physics, Aberystwyth University, Ceredigion, SY23 3BZ, UK 
dki-solar-telescope/csp/dkist-csp-workshops/). Although participation in those workshops was not mandatory for Science Use Case development, they served as an efficient way to acquaint the community with the telescope and instrument capabilities, the range of operational possibilities, and some of the challenges unique to ground-based observing at DKIST precision and scales. In turn, the DKIST project acquired a sense of the range and popularity of different observing configurations and an understanding of what will be required to meet the critical early scientific objectives.

This DKIST Critical Science Plan (CSP) is a snapshot of some of the scientific pursuits that the Daniel K. Inouye Solar Telescope hopes to enable as start-of-operations nears. The first-light DKIST images, released publicly on 29 January 2020 (www.nso.edu/inouye-solartelescope-first-light/), only hint at the extraordinary capabilities that will accompany full commissioning of the five facility instruments (see Rimmele et al., 2020). The CSP is an attempt to anticipate some of what those capabilities will enable. After a very brief synopsis of DKIST's capabilities, the article is divided into four broad sections: Magneto-convection and Dynamo processes, Flares and Eruptive Activity, Magnetic Connectivity through the Non-Eruptive Solar Atmosphere, and Long-Term Studies of the Sun, Special Topics, and Broader Implications. Each of these includes an introductory discussion followed by a more detailed exposition of specific research topics. The primarily focus is on issues to which DKIST will uniquely contribute. References are necessarily incomplete; they are exempli gratia only, even where not explicitly so noted.

\section{Unique DKIST Capabilities}

This section provides a very brief overview of the unique capabilities that DKIST will contribute. The full capabilities are discussed in detail in the accompanying articles (Rimmele et al., 2020; Davey et al., 2021; de Wijn et al., 2021; Fehlmann et al., 2021; Harrington et al., 2021; Jaeggli et al., 2021; Tritschler et al., 2021; von der Lühe et al., 2021; Wöger et al., 2021).

The DKIST primary mirror has a diameter of 4 meters, a size chosen so that small-scale plasma dynamics in the solar atmosphere can be well resolved, while simultaneously making

\footnotetext{
36 Bay Area Environmental Research Institute, NASA Research Park, Moffett Field, CA 94035, USA

Department of Astronomy, New Mexico State University, Las Cruces, NM 88003, USA

NASA Marshall Space Flight Center, Huntsville, AL 35812, USA

39 Catholic University of America, Washington, DC 20064, USA

40 NASA Goddard Space Flight Center, Greenbelt, MD 20771, USA

41 Institut d'Astrophysique Spatiale, CNRS, Université Paris-Saclay, 91405, Orsay, France

42 School of Mathematics and Statistics, University of St Andrews, St Andrews, Fife, KY16 9SS, UK

43 Space Science Center, University of New Hampshire, Durham, NH 03824, USA

Departamento de Física y Matemáticas, Universidad de Alcalá, 28871 Alcalá de Henares, Madrid, Spain 
polarization measurements of weak magnetic fields. The all-reflective, clear-aperture, offaxis optical configuration of the telescope allows broad wavelength access and minimizes scattered light, yielding the dynamic range sensitivity necessary for studies of the full solar atmosphere from the deep photosphere to $1.5 \mathrm{R}_{\odot}(0.5$ solar radii above the photosphere).

DKIST's first-light instrument suite consists of the Cryogenic Near-Infrared SpectroPolarimeter (CryoNIRSP: Fehlmann et al., 2021), the Diffraction-Limited Near-Infrared Spectropolarimeter (DL-NIRSP: Jaeggli et al., 2021), the Visible Broadband Imager (VBI: Wöger et al., 2021), the Visible Spectro-Polarimeter (ViSP: de Wijn et al., 2021), and the Visible Tunable Filter (VTF: von der Lühe et al., 2021). These five instruments will allow observations over the wavelength range 380 to $5000 \mathrm{~nm}$. With the exception of CryoNIRSP, they can be used individually or in combination, and employ a common integrated high-order adaptive-optics system (Rimmele et al., 2020), which enables diffraction-limited observations with resolution ranging from about 0.02 arcseconds at the shortest wavelength to 0.09 arcseconds at $1500 \mathrm{~nm}$. The stand-alone, cryogenically cooled CryoNIRSP will make seeing-limited observations with 0.15 arcsecond critical spatial sampling along the spectrograph slit over the spectral range 1000 to $5000 \mathrm{~nm}$. Scattering by the Earth's atmosphere, and thus the background sky-brightness, is generally low at the observatory site on Haleakalā, and is further reduced at long wavelengths. This will allow regular DKIST observations of the faint solar corona in the near to mid infrared. All instruments employ specially developed high-frame-rate, large-format cameras capable of non-destructive detector reads to improve signal to noise using longer exposures, and importantly, all instruments, with the exception of VBI (a narrow-band diffraction-limited filtergraph), are highly sensitive spectropolarimeters. Careful calibration of the instrument and telescope polarimetric contributions (Harrington, Sueoka, and White, 2019; Harrington et al., 2021, and the references therein) will allow measurement of magnetic flux densities of less than one Gauss both on and off the solar disk.

\section{Magneto-Convection and Dynamo Processes}

Magnetic fields on the Sun are highly structured and multi-scale. Field is found not only on the scale of active regions and sunspots but down to the smallest spatial scales observed. The small-scale fields may originate at larger scales and result as the endpoint of a turbulent cascade, or they may be induced directly by small-scale motions, perhaps in the photosphere itself, the result of a turbulent fluctuation dynamo (also called a small-scale or local dynamo). Many questions remain about the field distributions observed, about how and why the field is organized as it is in the solar photosphere.

On the largest scale, the global solar magnetic-activity cycle reflects dynamo processes that operate with remarkable regularity. The most conspicuous aspect of the global-scale dynamo is the cyclic appearance and disappearance of large areas of strong magnetic field organized into sunspots and active regions. These come and go with the solar cycle on spatial and temporal scales that suggest an origin in the deep convection zone or in the overshoot region just below it. But the solar convection zone is in a highly turbulent state and spans many pressure scale heights, with the density changing by a factor of over a million between the bottom and the top. If formed near the bottom of the convection zone, these magnetic structures must survive transit across this highly stratified and vigorously convecting region. Alternatively, active regions could re-form in the upper convection zone or in the photosphere itself. Although the vertical scale height in the photosphere is much smaller than the characteristic size of an active region, sunspot, or even a pore, some aspects of active-region 
evolution support this possibility, with the local dynamics displaying a complex interplay between the magnetic field and magneto-convective motions at all stages including formation and dissolution.

Sunspot umbrae are the most strongly magnetized regions of the Sun accessible to spectropolarimetric observations. They are cool and dark, characterized by magnetic fields that are close to vertical with respect to the photosphere, and exhibit constrained columnar convective motions that are only poorly understood. The surrounding penumbral field is highly inclined, with multiple interleaved magnetic components permeated by strong plasma flows. It is unknown how this multi-component sunspot structure arises. For example, reproduction of the observed penumbral properties by state-of-the-art radiative magneto-hydrodynamic simulations requires upper boundary conditions on the magnetic field whose counterparts on the Sun have yet to be identified. DKIST's large photon flux at high spatial and temporal resolution is critical to advancing our understanding of sunspot dynamics.

The quiet (non-active region) Sun is magnetized almost everywhere. In the quietest regions (inter-network) the fields appear to be weak and highly inclined. Determining the origin of these small-scale fields is difficult and DKIST will make critical contributions to this problem as well. It will enable simultaneous observations in Zeeman-sensitive and Hanle-sensitive lines, which can be used determine if the Hanle polarization signals measured in the inter-network regions of the Sun are consistent with the field deduced from Zeeman diagnostics. These comparisons will allow assessment of the amount of unresolved flux present as a function of resolution, and careful exploitation of these techniques will lead to precise measurement of the inter-network-field coverage and its spatial distribution as a function of global solar magnetic activity. The observed statistical properties and evolution of the small scale fields on the Sun can then be compared to those in dynamo simulations to untangle the signatures their origin.

Beyond its origin, knowledge of the small-scale magnetic-field distribution on the Sun is key to assessing the role of photospherically generated waves in atmospheric heating. Alfvén and magneto-acoustic waves propagate from the photosphere upward into the atmosphere, guided by local magnetic structures. The magneto-acoustic modes steepen, shock, and dissipate in the chromosphere or undergo mode conversion and make it to greater heights. The underlying magnetic-field distribution is critical to these processes and thus to the heat and momentum budget of the atmosphere. Small-scale fields also play an important role in modulating the solar radiative output. Small concentrations of magnetic field are sites of reduced gas pressure and density, and thus opacity. An amalgam of small-scale field elements reduces the average temperature gradient of a region, locally changing the radiative output and thus contributing to variations in global solar spectral irradiance. Many smallscale magnetic structures remain unresolved by current instruments, and changes in their size and contrast distributions with the solar cycle are unknown. Since weakly magnetized internetwork regions cover the bulk of the solar surface, currently unresolved field elements may play a significant role in global spectral-irradiance trends. DKIST will play a critical role in unraveling their contributions.

Several critical science topics in this research area are discussed in detail below, including i) the formation, structure, and dynamics of small-scale photospheric magnetic fields, ii) wave generation and propagation, iii) magneto-convective modulation of the solar luminosity, and iv) active-region evolution and sunspot fine structure.

\subsection{Small-Scale Photospheric Magnetic Fields: Formation, Structure, Dynamics}

Is there a small-scale dynamo operating in the solar photosphere? What is the relative importance of local amplification, flux emergence, flux cancelation, and non-local transport in 


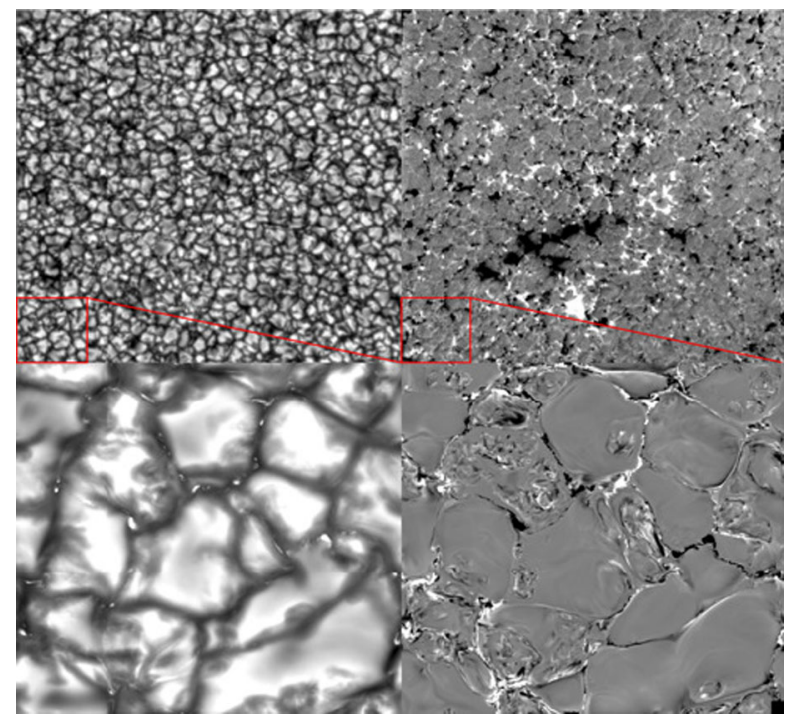

Figure 1 Top: Swedish Solar Telescope (Scharmer et al., 2003)/CRisp Imaging SpectroPolarimeter (Scharmer et al., 2008) observation of the quiet Sun with $0.15^{\prime \prime}$ resolution $(\approx 100 \mathrm{~km})$, displaying Stokes- $I$ and Stokes- $V$. Images courtesy L. Bellot Rubio (IAACSIC), S. Esteban Pozuelo (IAA-CSIC), and A. Ortiz (ITA, University of Oslo) www.esteast.eu/est/index.php?option=com_content \&view=article\&id=774\&lang=en?quiet-sun-magnetic-fields-

2-2-2. Red boxes are $6.1 \times 6.1 \mathrm{Mm}$, which is the size of the simulations below. Bottom: quiet-Sun simulation with 4-km grid spacing (comparable to an effective DKIST resolution of $20 \mathrm{~km}$ ) displaying bolometric intensity and vertical magnetic-field strength (in the range $\pm 200 \mathrm{G}$ ) at optical depth 0.1 . Simulation images from Rempel (2014), copyright by AAS.

small-scale field evolution? How turbulent are granular flows? How dependent is quiet-Sun magnetism on the solar cycle?

Convection in the solar photosphere is driven by rapid radiative losses from a thin layer that is less than a pressure scale height in vertical extent. In this layer, the internal, kinetic, and magnetic energy of the plasma are all comparable (within a factor of a few). This leads to vigorous dynamics with strong coupling between these three energy reservoirs. While the solar convection zone is generally believed to be a highly turbulent medium, this is less than obvious in observations of the solar photosphere (Nordlund et al., 1997). Plasma that is transported upward into the photosphere, and is observed as bright hot granules, has undergone significant horizontal expansion and is expected to show nearly laminar flow structure even at DKIST resolution. A higher degree of turbulence may be found in the intergranular downflow lanes, but in these regions it likely develops as an advected instability at the interface between upflows and downflows as the fluid moves downward out of the photospheric boundary layer. Turbulence may thus only be apparent in high-spatial-resolution measurements of the deep photosphere, and then only with the anticipated high contrast achievable by DKIST.

Small-scale magnetic field is ubiquitous in the solar photosphere (Figure 1) and, in terms of unsigned flux density, it exceeds the total active-region magnetic field at all phases of the solar cycle (e.g. Lites et al., 2008; de Wijn et al., 2009; Lites, 2011; Bellot Rubio and Orozco Suárez, 2019). In quiet-Sun regions, the field has an average strength of about onethird the value it would have in equipartition with the kinetic energy of the flows (mean 
field strengths of about 200 G: Danilovic, Schüssler, and Solanki, 2010), but since most of the field is concentrated into small-scale elements in the downflow lanes (e.g. Bellot Rubio and Orozco Suárez, 2019), the field strength reaches much higher local values and strong feedback on the convection is expected. A detailed understanding of the interaction between the flow and the small-scale magnetic field, and the possible role of the field in the suppression of turbulence within intergranular downflows, is still elusive due to the resolution limits of current observations. This has broader significance, as the development of turbulence in the shear layers of intergranular downflow lanes may contribute to spectral-line broadening (Section 6.2). Furthermore, even in the quiet Sun, a small fraction of the magnetic field is locally amplified to a strength of a few kilogauss. This may occur through the convective-collapse process (Parker, 1978; Webb and Roberts, 1978; Spruit, 1979; Spruit and Zweibel, 1979; Grossmann-Doerth, Schüssler, and Steiner, 1998), with some observational evidence for that mechanism (e.g. Hewitt et al., 2014), although significant uncertainty remains (Venkatakrishnan, 1986; Bushby et al., 2008; Borrero et al., 2017). Only the largest of the kilogauss elements are resolved with current instrumentation. DKIST will allow dynamical studies of the formation and evolution of individual kilogauss elements and detailed statistical analysis of the quiet-Sun flux concentration size and strength distributions and their solar-cycle dependencies. Since modulation of the solar radiative output by magnetic flux elements is the primary cause of solar-irradiance variations, these studies will also be critically important in that context (see Section 3.3 below).

Beyond the quiet Sun, numerous small-scale magnetic loop-like structures are found in regions that are unipolar on larger scales, including both active-region plage (Wang, 2016; Wang, Ugarte-Urra, and Reep, 2019) and coronal holes (Wang, Warren, and Muglach, 2016). While difficult to discern in magnetograms, where minority-polarity signatures are often absent, these structures are readily apparent in EUV images. They have horizontal scales of $\approx 2-5 \mathrm{Mm}$ and evolve on timescales of minutes. In Solar Dynamics Observatory/Atmospheric Imaging Assembly (SDO/AIA) images of active-region plage, the structures sometimes show an inverted-Y configuration, suggestive of magnetic reconnection. This role is supported by their presence in the cores of bright coronal plumes, again even when no minority-polarity flux is visible in photospheric magnetograms, where they appear to cluster and contribute to coronal-plume energization, heating, and emission when flow convergence causes interchange reconnection between them. Reflecting this dynamic, coronal-plume emission changes on timescales of hours to a day as the supergranular flow field evolves (Wang, Warren, and Muglach, 2016; Avallone et al., 2018; Qi et al., 2019). Previous magnetogram-based studies (e.g. Hagenaar, DeRosa, and Schrijver, 2008) measured ephemeral region emergence rates inside unipolar regions that were at least a factor of three lower than those in the quiet Sun. The discovery of ubiquitous small-scale loops with very weak if any minority-polarity signatures suggests that magnetograms may significantly underestimate the amount of mixed-polarity flux in plage, strong network, and coronal-hole regions. That mixed-polarity flux may play an important role in coronal heating as it reconnects with the large-scale overlying fields, both in active regions (Wang, 2016; Wang, Ugarte-Urra, and Reep, 2019) and in coronal holes where it may also help to drive the solar wind (Wang, Warren, and Muglach, 2016). High-resolution, high-sensitivity magnetic-field measurements with DKIST will be able to quantitatively assess this suggestion.

The small-scale magnetic field in the solar photosphere is maintained by a combination of several processes: i) dispersal of active region flux; ii) turbulent amplification by photospheric flows; and iii) small-scale flux emergence from deeper regions. While process i) reflects contributions from the large-scale global dynamo, processes ii) and iii) are linked to a turbulent-fluctuation dynamo that relies on the chaotic nature of flows in the uppermost 
layers of the convection zone. Some numerical simulations of turbulent-fluctuation dynamos in the solar context have been successful in generating small-scale magnetic field with flux densities similar to those suggested by current observations, but these and all numericalfluctuation dynamo models operate at magnetic Prandtl numbers (ratio of viscosity and magnetic diffusivity) close to unity (e.g. Cattaneo, 1999; Vögler and Schüssler, 2007; Rempel, 2014; Khomenko et al., 2017). By contrast, the magnetic Prandtl number in the solar photosphere, if based on molecular values of the diffusivities, may be as low as $10^{-5}$. Producing a fluctuation dynamo at small magnetic Prandtl number and large Reynolds number, the parameter regime most relevant to the Sun, is an unmet challenge for both numerical simulations and laboratory experiments. This makes the solar photosphere a unique plasma laboratory.

Moreover, even Prandtl-number-unity models of the solar-fluctuation dynamo can generate total unsigned magnetic-flux densities close to those observed only when the advection of flux from deeper layers is allowed (Rempel, 2014), not if the dynamo is strictly local, operating only in the photosphere. This suggests that the observed level of quiet-Sun magnetism requires a significant amount of magnetic-field recirculation within the convection zone, with convective motions bringing substantial amounts of field up into the photosphere from below (Stein, Bercik, and Nordlund, 2003; Rempel, 2014, 2018). The behavior of the solar dynamo across many coupled scales may thus be critical to its operation (e.g. Charbonneau, 2016; Wright and Drake, 2016; Beaudoin et al., 2016), and a more complete understanding of the solar fluctuation-dynamo may require quantitative assessment of the relative importance of local field amplification and non-local field transport (see, e.g. Gošić et al., 2016, for recent observational efforts).

Global-scale transport of small-scale field is already a central ingredient of many global dynamo models (see reviews by Charbonneau, 2014; Cameron, Dikpati, and Brandenburg, 2017), with the accumulation of small-scale field elements of a dominant polarity in the polar regions critical to the reversal of the global dipole moment. For this reason, the measured solar polar field strength is often cited as the most reliable indicator for solar-cycle predictions (e.g. Bhowmik and Nandy, 2018). The behavior of the small-scale polar fields can be directly assessed via synoptic DKIST observations (Section 6.1), and the flux transport that results from supergranular diffusion and meridional circulation can be better quantified by high-resolution DKIST studies of magnetic-element displacement statistics (Agrawal et al., 2018). Relatedly, global-scale/long-period modulation of macrospicules has been reported (e.g. Kiss, Gyenge, and Erdélyi, 2017; Kiss and Erdélyi, 2018), and spicules and other chromospheric dynamical events are key targets for further DKIST study (Section 5.1). DKIST will thus enable quantitative assessment of the small-scale field evolution which may underlie the global behavior.

\subsection{Acoustic-Gravity Wave Excitation}

What excites the solar acoustic oscillations? How do observed source properties effect helioseismic inferences? What physics underlies flare-induced acoustic wave emission?

Magnetohydrodynamic wave generation, propagation, mode conversion, and dissipation are central processes in the energy and momentum budget of the solar atmosphere. Those issues are largely discussed in Section 5.4. Here we focus on the role of DKIST in determining the source of the solar $p$-modes and the potential for gravity-wave observations and diagnostics in the lower solar atmosphere.

Acoustic-mode excitation on the Sun, and other stochastically excited stars, likely results from small-scale dynamical processes associated with convection (Figure 2), but the 
Figure 2 Filtered Doppler velocity (left) and a simultaneous continuum image of the same area (right) from the first flight of the IMaX instrument on the Sunrise balloon-borne observatory. Time series of these images suggest the launching of parallel wavefronts (center of lefthand image) by new downflow-lane formation during granule fragmentation.

From Roth et al. (2010), copyright by AAS.

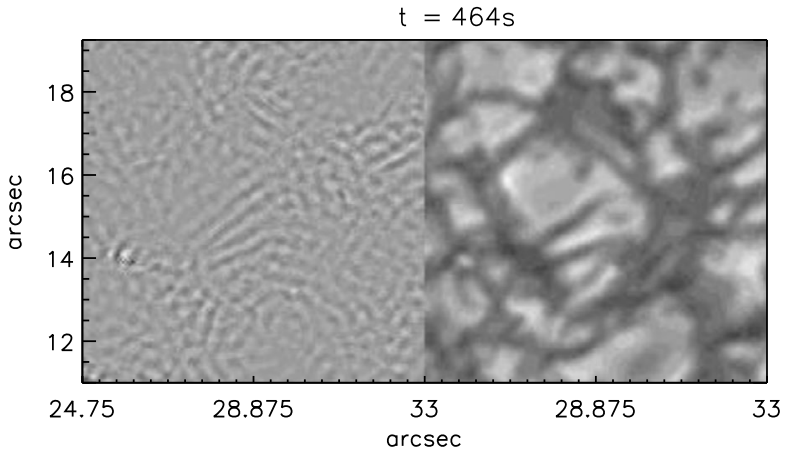

detailed source properties are not known. Some theoretical work points to turbulent excitation dominated by Reynolds-stress induced pressure fluctuations, the Lighthill mechanism (Lighthill, 1952; Goldreich and Keeley, 1977; Goldreich and Kumar, 1990; Balmforth, 1992a,b), possibly occurring within intergranular lanes, while other work suggests that the dominant source is associated with pressure perturbations caused by local radiative cooling in the photosphere (Stein and Nordlund, 1991; Goldreich, Murray, and Kumar, 1994; Rast, 1999) and the sudden formation of new downflow plumes, with the latter process often occuring during the fragmentation of large granules (Rast, 1995). Additionally, some flares are strong acoustic-wave sources (e.g. Kosovichev and Zharkova, 1998; Ambastha, Basu, and Antia, 2003; Donea and Lindsey, 2005), and the mechanisms associated with acoustic emission during flaring is also only partially understood (Lindsey et al., 2014).

There is observational support for both of the hydrodynamic mechanisms suggested above (Rimmele et al., 1995; Chaplin et al., 1998; Goode et al., 1998; Straus et al., 1999; Skartlien and Rast, 2000; Severino et al., 2001; Bello González et al., 2010; Roth et al., 2010), but it is unclear which process dominates, how much power is radiated by each, and how well that power is coupled to the solar $p$-modes. Unambiguous characterization of the $p$-mode sources requires separating local wave motions from higher-amplitude compressible convective flows and wave coherence patches. This is theoretically challenging, and observationally likely requires high spatial and temporal resolution observations (on the order of tens of kilometers at 15-second cadence) over a range of heights from the deep to middle photosphere. A statistically significant number of individual events must be studied to assess their physical characteristics and energy contributions, and thus their importance, and it is only with DKIST that the required observational capabilities will become regularly available. Moreover, direct measurement of pressure fluctuations along with the temperature and velocity fields are needed to fully characterize the excitation mechanisms, and pressuresensitive spectral diagnostics are only now being developed in preparation for DKIST start of operations.

The precise nature of the $p$-mode excitation events, their efficiency, phasing, and relative importance to mode excitation, is critical to both local and global helioseismic inferences. For global helioseismology, and local methods that employ the mode spectrum such as ring-diagram analysis (Haber et al., 2002; Gizon and Birch, 2002, and the references therein), precise determination of the modal frequencies and frequency shifts depends on the assumed power-spectral-line shape with which the modes are fit. One source of systematic error in the measured solar $p$-mode frequencies is the unknown non-Lorentzian shape of the spectral lines. Line profiles vary with wavenumber and frequency depending on the source depth and physical properties (e.g. Gabriel, 1992; Duvall et al., 1993; Gabriel, 1995; 
Rast and Bogdan, 1998; Nigam et al., 1998; Skartlien and Rast, 2000). Similarly, local helioseismological deductions are sensitive to the phase relationship between the waves and their source. For example, travel-time kernels used in time-distance helioseismology depend on the assumptions about the source phasing and characteristics (Gizon and Birch, 2002; Birch, Kosovichev, and Duvall, 2004). Source properties may be particularly critical for multi-height local helioseismology if the source is spatially and temporally extended, as it is likely to be.

Additionally, the local acoustic wave field can be used to diagnose magnetic structures ranging in size from small scale elements to sunspots. Mode interaction and conversion at the interface of a magnetized region leads to a range of signatures in the photosphere including wave absorption, mode conversion, and scattering (with associated phase shifts and amplitude changes) that can be used to reveal the subsurface (e.g. Braun, Duvall, and Labonte, 1987, 1988; Spruit and Bogdan, 1992; Braun et al., 1992; Cally, Bogdan, and Zweibel, 1994; Braun, 1995; Cally and Bogdan, 1997; Gizon, Birch, and Spruit, 2010; Liang et al., 2013; Waidele and Roth, 2020, and the references therein) or overlying structure of the field (e.g. Finsterle et al., 2004a,b; Jess et al., 2020, and Section 5.4 below). The combination of new methods for local source identification (Bahauddin and Rast, 2021) and multi-height high spatial and temporal resolution spectropolarimetric measurements with DKIST promises a new era for helioseismic diagnosis of sunspots and smaller-scale magnetic structures.

Finally, the photosphere is the lower boundary of the stably stratified solar atmosphere, and convective overshoot into the lower solar atmosphere drives internal acoustic-gravity waves. These waves probe the physical properties of the layers above and may make a significant contribution to the solar chromosphere energy balance (e.g. Hindman and Zweibel, 1994; Straus et al., 2008). The increased spatial resolution and temporal cadence of DKIST will allow higher spatial and temporal frequency waves to be observed, and the extended high-quality observation periods anticipated will enhance the frequency resolutions achievable. This may allow individual atmospheric gravity-wave-mode identification and detailed study of their convective driving. Gravity-wave excitation is a process that is broadly important in regions more difficult to observe, such as the base of the solar convection zone and in the interior of other stars, and observations of the photosphere and lower solar atmosphere are critical to constraining the underlying excitation processes. Moreover, since the solar atmosphere is highly magnetized, significant mode coupling occurs as the waves propagate upward, and as this coupling depends on the orientation and strength of the local magnetic field, such observations have immediate diagnostic value (Vigeesh, Jackiewicz, and Steiner, 2017; Vigeesh et al., 2019, Section 5.4 below).

\subsection{Magneto-Convective Modulation of the Solar Irradiance}

How do small-scale magnetic-flux elements contribute to global solar-irradiance variations? How well do observed temperature and pressure stratifications within flux elements agree with atmosphere models employed for irradiance reconstruction?

Observational evidence suggests that solar irradiance is modulated by changes in solar surface magnetism. Based on this hypothesis, empirical techniques have been developed to reproduce total and spectral solar-irradiance variations from observed changes in the coverage of magnetic features over the solar disk. Depending on their size and field strength, different magnetic features show different center-to-limb variation in different spectral regions. Thus both the disk location and disk coverage of the features are needed to model irradiance changes, and full-disk moderate resolution (one to two arcsecond) observations 

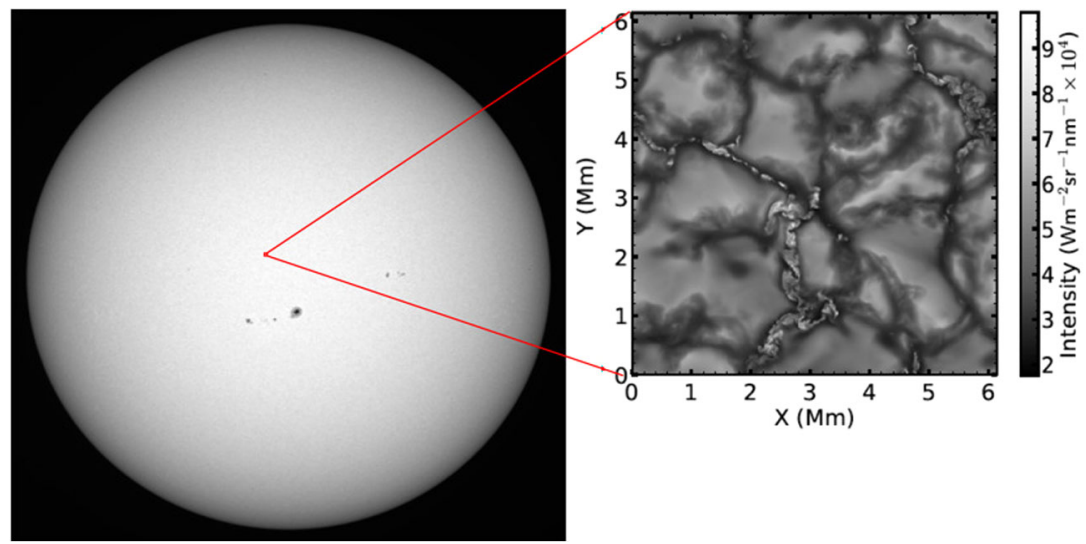

Figure 3 Full-disk images are used in solar-irradiance models because magnetic structure contributions depend on disk position. There are significant differences in the magnetic morphology underlying pixels classified as the same structure at full-disk resolution. These influence solar spectral irradiance and its variability with solar cycle. Image on left from the Precision Solar Photometric Telescope (image courtesy M.P. Rast, lasp.colorado.edu/pspt_access/). Image on right from Rempel (2014).

are typically employed (e.g. Chapman, Cookson, and Dobias, 1996; Krivova et al., 2003; Yeo et al., 2017).

However, both high-spatial-resolution (sub-arcsec) observations and radiative magnetohydrodynamic simulations indicate the presence of magnetic structures too small to be detected at moderate resolution (Figure 3). Spectra modeled on the basis of full-disk moderateresolution-image features do not necessarily capture the radiative output of the underlying highly structured atmosphere, nor do they distinguish between differently structured atmospheres with the same lower-resolution appearance (Röhrbein, Cameron, and Schüssler, 2011; Criscuoli, Norton, and Whitney, 2017; Peck et al., 2019). The mapping between fulldisk imagery, the underlying small-scale structure, and spectral irradiance is only poorly understood butis critical for accurate irradiance modeling, particularly spectral-irradiance modeling (see Ermolli et al., 2013, and the references therein). There thus remains significant uncertainty about whether full-disk magnetic-structure-based irradiance models can account for the observed spectral-irradiance variations. In particular, both the sign and the magnitude of the spectral-irradiance trends with solar cycle are controversial, with some authors reporting out-of-phase irradiance variation in key wavelength bands (Harder et al., 2009) and others reporting in-phase variation across the spectrum (Wehrli, Schmutz, and Shapiro, 2013). While out-of-phase variations have been explained in terms of a change in the mean photospheric temperature gradient with cycle, which atmospheric components contribute most to that mean change remains unclear, particularly as absolute irradiance measurements are disk integrated. Addressing these uncertainties requires developing both an understanding of the statistical distributions of small-scale elements and reliable techniques for measuring the photospheric temperature gradient (Faurobert, Balasubramanian, and Ricort, 2016; Criscuoli and Foukal, 2017).

Unresolved magnetic elements are particularly important to the spectral output of the quiet Sun (Schnerr and Spruit, 2011) and its center-to-limb profile (Peck and Rast, 2015). It is against this that the contrasts and contributions of magnetic structures are often measured. The quiet Sun covers the majority of the solar photosphere and typically its integrated irradiance contribution is taken to be constant in time, but due to the presence of possibly 
time-varying unresolved magnetic structures this may not be the case. Measuring how the magnetic substructure of the quiet Sun actually changes with the solar cycle is a key DKIST capability. Beyond this, the radiative contributions of magnetic structures such as plage and faculae depend on the fact that they are structured on unresolved scales (Okunev and Kneer, 2005; Criscuoli and Rast, 2009; Uitenbroek and Criscuoli, 2011). Some facular pixels show negative continuum contrast in full-disk images even close to the limb. This may reflect uncertainty in the quiet-Sun profile against which the contrast is measured or underlying substructure in the faculae themselves (e.g. Topka, Tarbell, and Title, 1997). Measuring the radiative properties of composite features and understanding the physical mechanisms that determine their spectral output is an important next step in the development of irradiance reconstruction and modeling techniques. Models must be capable of statistically accounting for contributions from a distribution of small-scale magnetic features unresolved in full-disk images.

Finally, the astrophysical implications of small-scale fields extend beyond the Sun. The spectral-energy distribution of stars is a key fundamental input in the modeling of planetary atmospheres (e.g. Hu, Seager, and Bains, 2012; Miguel and Kaltenegger, 2014). In particular, UV radiation is responsible for the production and destruction of molecular species that are the anticipated biomarkers to be used in future exoplanet atmospheric characterization missions. More critically, UV variability is important in determining the climate and habitability of (e.g. Scalo et al., 2007; France et al., 2013; Linsky, France, and Ayres, 2013; O'Malley-James and Kaltenegger, 2019) and biogenic processes on (Buccino, Lemarchand, and Mauas, 2007) extrasolar planets. While not sensitive to UV radiation directly, DKIST observations of the Sun can be used to develop, constrain, and test models of stellar chromospheres and/or proxies to reliably reconstruct UV spectra from measurements obtained at longer wavelengths. Moreover, accounting for magnetic modulation of stellar brightness is crucial to the detection and correct interpretation of stellar light curves and their use in exoplanet characterization (e.g. Apai et al., 2018; Rackham, Apai, and Giampapa, 2019). DKIST's ability to probe the physical mechanisms underlying stellar brightness variations will thus contribute to both stellar astrophysics and the modeling of host-star contributions to exoplanet transit photometry and spectroscopy.

\subsection{Active Region Evolution and Sunspot Fine Structure}

Why sunspots? Why is magnetic flux concentrated into sunspots? How do they form? Why do they have penumbrae? What is the dynamical and magnetic substructure of sunspot umbrae and penumbrae, and what are the magnetic and dynamical links to the chromosphere, transition region, and corona above?

Sunspots are the most prominent manifestation of strong magnetic fields in the solar atmosphere. They consist of a central dark umbra, within which the magnetic field is primarily vertically oriented, and a brighter penumbra, with weaker and more inclined fields. Both regions exhibit small-scale structure, such as umbral dots and penumbral filaments, and rich dynamics with mass flows and wave motions spanning the photosphere and chromosphere. The range of observed behaviors reflects the presence of magnetic fields of varying strengths and inclination angles, making sunspots ideal for studying magneto-convective processes in complex magnetic-field geometries.

However, despite significant advances over the past decades, observationally characterizing the field geometry and associated flow dynamics in sunspot umbrae and penumbrae presents formidable challenges. These are due to small scales present, the low umbral photon counts, and the difficulties associated with interpreting the distorted spectral-line profiles 

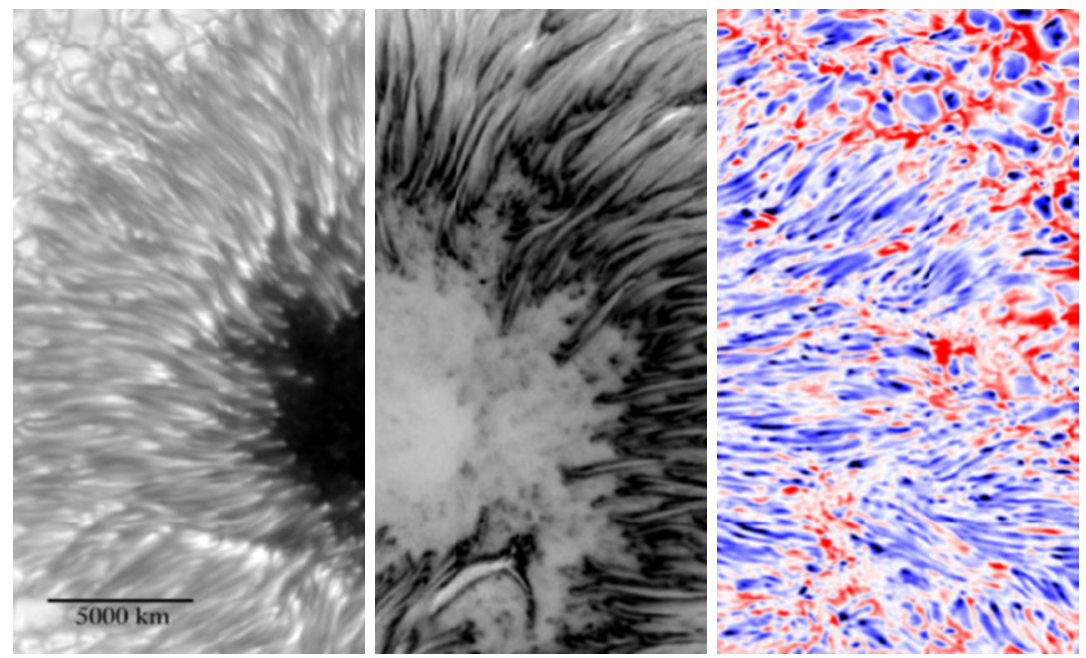

Figure 4 Sunspot in Active Region 11302. Observed in intensity (left), circular polarization (middle), and line-of-sight velocity (right) with the CRisp Imaging Spectro-Polarimeter (Scharmer et al., 2008) at the Swedish Solar Telescope (Scharmer et al., 2003) on 28 September 2011. Penumbral fine structure is apparent in all three maps, showing details down to the diffraction limit of the telescope $(0.15 \operatorname{arcsec})$. Lateral edges of penumbral filaments appear to harbor localized short-lived vertical downflows (shown red). Adapted from Esteban Pozuelo, Bellot Rubio, and de la Cruz Rodríguez (2015, 2016).

observed (Borrero and Ichimoto, 2011; Rempel and Schlichenmaier, 2011; Hinode Review Team et al., 2019). As a result, we do not yet know the magnetic topology or flow structure of the penumbra at the smallest scales, nor how these vary with height in the atmosphere. In state-of-the-art numerical models (e.g. Heinemann et al., 2007; Rempel, Schüssler, and Knölker, 2009; Rempel, 2011, 2012a,b), penumbral filaments reflect overturning convection in highly inclined magnetic-field regions, with the Evershed flow being the main flow component oriented in the radial direction. These models predict the existence of vertical downflows at the edges of penumbral filaments where the overturning plasma descends below the solar surface, but systematic study of such downflows on the Sun is very difficult due to their intrinsically small sizes (e.g. Esteban Pozuelo, Bellot Rubio, and de la Cruz Rodríguez, 2015, 2016, and the references therein). Supersonic vertical velocities at the outer end of penumbral filaments have been reported (Figure 4) and can be associated with penumbral field lines dipping down below the solar surface (Tiwari et al., 2013; van Noort et al., 2013; Esteban Pozuelo, Bellot Rubio, and de la Cruz Rodríguez, 2016), but similar flows have also been detected in the inner penumbra without relation to the Evershed flow (Louis et al., 2011). In both regions, very large field strengths (on the order of $7 \mathrm{kG}$ and $5 \mathrm{kG}$, respectively, compared to typical peak umbral values of $2-3 \mathrm{kG}$ ) have been associated with the high-speed flows (Siu-Tapia et al., 2017; Okamoto and Sakurai, 2018), but because the observed Stokes profiles show anomalous shapes, these determinations are uncertain. Multi-line, very high-spatial-resolution, spectropolarimetric observations by DKIST will allow improved inference of both the field strengths and flow velocities and clarify the origin of the supersonic flows and the processes leading to local field amplification beyond umbral values.

More fundamentally, the formation of the penumbra itself remains a mystery (e.g. Rezaei, Bello González, and Schlichenmaier, 2012). The process lasts only a few hours, making it difficult to capture, and observations have not yet identified the mechanism triggering the 
process around a naked pore. There are indications that the chromospheric magnetic field may play a prominent role. In simulations (Rempel, 2012a,b) sunspots only form an extended penumbra if the field inclination at the top boundary is artificially enhanced. The nature and origin of a similar field on the Sun, if it exists, is unknown. Penumbrae are a robust feature of sunspots and are present under a wide range of conditions, whereas the presence of penumbrae in sunspot simulations requires this special boundary condition. This critical difference may be related to the fact that the dynamics of a penumbra during formation are observationally very different from those seen once it is mature. Instead of a regular Evershed outflow, "counter" Evershed inflows, toward the umbra, are observed during formation. These inward-directed flows turn into the classical radial outward Evershed flow as the penumbra stabilizes (Schlichenmaier, González, and Rezaei, 2011). This important transition and other chromospheric precursors (Shimizu, Ichimoto, and Suematsu, 2012; Murabito et al., 2017) likely hold clues about the penumbral formation process, a process missing from the simulations. High-sensitivity DKIST measurements of the vector magnetic field and flow in both the photosphere and the chromosphere during penumbra formation will allow an assessment of the relative importance of photospheric magneto-convective processes and the evolution of overlying chromospheric magnetic fields.

In umbrae, strong magnetic fields severely constrain convective motions, and numerical simulations suggest that umbral dots are the signature of convective plumes (Schüssler and Vögler, 2006). Direct observational confirmation is difficult due to the small sizes and low contrast of the structures as well as the overall low photon counts in sunspot umbrae. These conspire to dramatically reduce the signal-to-noise ratio of the observations, particularly when spectropolarimetric measurements are needed to determine the magnetic-field configuration along with the flow. Interestingly, umbral dots may not be uniformly distributed throughout the umbra, with recent measurements suggesting the existence of very dark, fully non-convective umbral regions (Löhner-Böttcher et al., 2018). DKIST's off-axis design and consequent low scattered-light properties will allow high-precision high-resolution Doppler and spectropolarimetric measurements of even the darkest regions of the umbra.

The magnetic topology and dynamics of the chromosphere above sunspots are also poorly understood. Few high-spatial-resolution observations extending from the photosphere up into the chromosphere with the required sensitivity have been made, so the connectivity with height is uncertain. Nonetheless, intense chromospheric activity is observed above sunspots on small spatial scales. Examples include short dynamic fibrils, umbral and penumbral microjets, penumbral bright dots, and jets at the edges of light bridges (e.g. Katsukawa et al., 2007; Louis et al., 2008; Shimizu et al., 2009; Rouppe van der Voort and de la Cruz Rodríguez, 2013; Yurchyshyn et al., 2014; Louis, Beck, and Ichimoto, 2014; Tian et al., 2014; Alpert et al., 2016; Robustini et al., 2016; Tian et al., 2018). These phenomena likely involve shocks and/or magnetic reconnection, and they are expected to be a source of chromospheric heating (e.g. Bard and Carlsson, 2010; Felipe, Khomenko, and Collados, 2011; de la Cruz Rodríguez et al., 2013; Henriques et al., 2017; Nelson et al., 2017b), and they may have far-reaching effects, up into the overlying transition region and corona (e.g. Samanta et al., 2017; Tian et al., 2018, and the references therein), with some penumbral micro-jets (perhaps those that are particularly large) displaying signatures at transition-region temperatures (Vissers, Rouppe van der Voort, and Carlsson, 2015; Tiwari et al., 2016).

Studying the dynamic chromosphere above sunspots is challenging. Low photon counts pose even more of a difficulty for observations of chromospheric features than they do for studies of the umbral photosphere. The finely structured faint absorption features that are apparent as dark fibril-shaped inhomogeneities within chromospheric umbral flashes (e.g. Socas-Navarro et al., 2009; Henriques et al., 2015) are so small and faint that they can only 
be observed with great difficulty using pre-DKIST telescopes and instrumentation. Even emission features are difficult to observe with spatial and temporal resolutions sufficient to distinguish the responsible physical mechanisms. For example, much of the spatial and temporal structuring of umbral micro-jets that was originally thought to be reconnection related (Bharti, Hirzberger, and Solanki, 2013; Nelson et al., 2017b) may instead be the signature of a shock/compression front forming in the corrugated sunspot atmosphere, with the time varying emission reflecting local density/velocity structures that enhance or delay the shock along a steepening compression front (Henriques et al., 2020). DKIST will enable spectropolarimetric measurements of such dim small-scale features with sufficient polarimetric sensitivity to constrain their physical properties. In combination with tools such as inversion-based semi-empirical modeling (e.g. de la Cruz Rodríguez et al., 2013) and numerical simulation, DKIST will advance our fundamental understanding of these phenomena and enable their use as diagnostics of the three-dimensional physical conditions at the event sites.

The transition region above sunspot umbrae poses additional observational challenges. It is also highly dynamic and structured at very small scales, with fast downflows and coincident strong emission, and that emission typically occurs at the short wavelengths characteristic of both transition-region and upper-chromospheric temperatures and densities (e.g. Chitta, Peter, and Young, 2016; Samanta, Tian, and Prasad Choudhary, 2018; Nelson, Krishna Prasad, and Mathioudakis, 2020). Nonetheless, it has been demonstrated that groundbased observations of sufficient spatial and temporal resolution can be combined with shortwavelength spaced-based measurements to improve transition region inversions (de la Cruz Rodríguez, Leenaarts, and Asensio Ramos, 2016; Bose et al., 2019). Simultaneous highcadence DKIST multi-line time series combined Interface Region Imaging Spectrograph (IRIS), Hinode, Solar Orbiter (SO), and SDO observations will help clarify the relationships between the rich chromospheric dynamics above sunspots and the heating of the overlying solar transition region and corona.

Finally, the disappearance and dissolution of sunspots is not well understood. Highcadence, high-resolution magnetogram sequences are required to assess and quantify the role of small moving magnetic features, which stream out from the penumbral border and merge with the surrounding network magnetic field during sunspot dissolution (e.g. Hagenaar and Shine, 2005). While these features seem to be associated with the slow disaggregation of the sunspot magnetic bundle, only limited measurements of their individual magnetic topologies, configurations, and flux, and thus of their integrated contributions to sunspot decay, have been made (e.g. Kubo, Shimizu, and Tsuneta, 2007).

\section{Flares and Eruptive Activity}

Solar flares and coronal mass ejections are spectacular phenomena that are not only critical to the space-weather environment of Earth, but reflect processes ubiquitous in astrophysical plasmas. The Sun, by its proximity, allows the unique possibility of high-resolution studies of the physics of magnetic reconnection, the related acceleration of particles to relativistic energies, the heating of the plasma to more than 10 million Kelvin, and the excitation of plasma waves. Flares and mass-ejection events are a template for magnetic activity on a variety of stars, some much more active than the Sun, with implications for the habitability of exoplanets. Moreover, the underlying physical processes are common to even more energetic astrophysical events that produce relativistic jets, beams, and shocks. 
The solar atmosphere is a dynamic magnetized plasma of very high electrical conductivity that can form and sustain complex current systems. When these currents abruptly reconfigure during magnetic-field reconnection events, particle acceleration and bulk plasma ejection can result. Intense electromagnetic radiation occurs when the energy of accelerated non-thermal particles or accompanying plasma waves is deposited and thermalized. Observationally, solar flares are transient bursts of electromagnetic radiation over a wide range of wavelengths, from radio to X-ray (or even shorter in some cases). Coronal mass ejections (CMEs) occur when a magnetic-flux system with substantial magnetic free energy, initially confined by overlying field, evolves so that the outward magnetic pressure-force on the trapped plasma overcome the inward magnetic-tension force of the overlying fields. The energetic flux system can then escape into interplanetary space, with the consequent expulsion of magnetized coronal plasma into the heliosphere. Subsequent flaring often occurs as the magnetic field in the wake of the coronal mass ejection relaxes back to a lower energy state. Many flares occur without CMEs, and some CMEs occur without substantial enhancement of radiative emission, but it is common for both phenomena to occur as part of a solar eruptive event.

Much of the research on solar flares and CMEs over the last decades has focused on the evolution of temperature and density structures within the corona. DKIST's ability to regularly and systematically measure coronal magnetic fields will enable more direct evaluation of the connections between the local plasma properties, the radiative signatures of flares and CMEs, and the field evolution. The ability of DKIST to readily probe the lower solar atmosphere at very high spatial resolution will allow determination of the physical conditions deep in the atmosphere during flaring and eruptive events, and DKIST measurements of subtle changes in the intensity and topology of the photospheric magnetic fields underlying flaring regions will help in assessing the amount of magnetic energy released during these events. Spatially resolved rapid imaging and spectroscopy of the chromospheric foot points of flares will allow determination of the actual extent of the sites of flare-energy deposition, providing strong constraints on particle-acceleration mechanisms. Determination of the penetration depth of the flare disturbance into the lower atmosphere, facilitated by DKIST's multi-wavelength capabilities, will help define the roles of proposed energytransport mechanisms and the relative importance of waves and particle beams. Together these capabilities will elucidate the mechanisms that underly the rapid conversion and deposition of the energy stored in stressed magnetic fields during flaring and plasma-ejection events.

Several critical science topics in this research area are discussed in detail below, including i) flare and CME precursors, ii) changes in the magnetic field associated with flares and CMEs, iii) energy deposition during flares, and iv) the fundamental structure and evolution of flare ribbons.

\subsection{Flare and Coronal Mass Ejection (CME) Precursors}

What triggers coronal mass ejections and solar flares? What are the relative importance of mechanisms proposed, such as flux emergence, current-sheet instabilities, and small-scale flux cancellation? When, where, and how do large-scale flux ropes form?

Solar flares and coronal mass ejections result when magnetic energy stored in the solar corona is released. The release is sudden (over a few to tens of minutes) compared to the timescale over which energy is built up and stored (many hours to days, e.g. Schrijver, 2009). Despite decades of study, the physical mechanisms that trigger flares and/or CMEs remain 


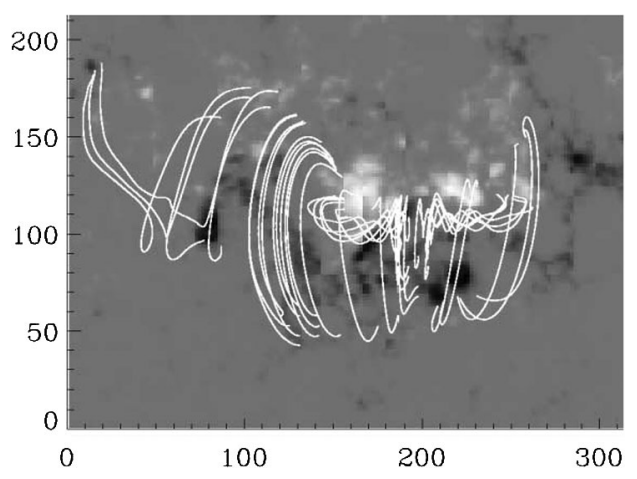

Figure 5 Nonlinear force-free magnetic-field reconstruction of NOAA Active Region 9077 before the X5.7/3B (10:24 UT) flare on 14 July 2000 (SOL2004-714T1024), based on vector magnetograms taken at the Huairou Station of the Beijing Astronomical Observatory. The photospheric longitudinal magnetogram is shown in the background for reference. Field lines trace the magnetic flux-rope above the neutral line and the overlying arcade field. Axes are marked in units of arcseconds. From Yan et al. (2001), copyright by AAS.

elusive. Understanding the conditions that lead up to a CME or flare and identifying the trigger mechanisms that initiate them are central to developing the predictive capabilities necessary for successful forecasting of their space weather impacts.

While no "necessary and sufficient" conditions for flaring or CME initiation have been identified, various precursor phenomena have been reported to occur in chromospheric and coronal observations in the minutes to hours preceding flares or CMEs (for reviews see, e.g., Chen, 2011; Shibata and Magara, 2011; Green et al., 2018). In addition to possible magnetic-field and flow diagnostics (e.g. Park et al., 2018; Kontogiannis et al., 2019; Korsós et al., 2020b, discussed in Section 4.2), these include multiple small-scale brightenings in $\mathrm{H} \alpha$ (Martres, Soru-Escaut, and Nakagawa, 1977; Druett et al., 2017) and/or soft xray (e.g. Tappin, 1991), plasmoid ejection (e.g. Hudson, 1994; Kim et al., 2009), S-shaped or inverse-S (sigmoidal) soft X-ray emission (Canfield, Hudson, and McKenzie, 1999), and the slow rise and darkening of chromospheric filaments (filament activation: e.g. Martin, 1980; Schuck et al., 2004). There is also some evidence for spectral-line broadening before flares (e.g. Harra et al., 2009; Woods et al., 2017) and pre-flare thermal X-ray enhancements without evidence of non-thermal particles (Benz, Battaglia, and Güdel, 2017).

The limited spatial resolution, cadence, and wavelength coverage of many previous observations have hampered efforts to understand the physical nature of these potential precursor signals, but recent high-resolution spectropolarimetric observations with the 1.6$\mathrm{m}$ Goode Solar Telescope suggest that very small-scale polarity inversions, currents, and magnetic loops in the low atmosphere are associated with the main flare initiation (Wang et al., 2017a). Magnetohydrodynamic simulations also show that the introduction of small opposite-polarity bipoles or reversed-magnetic-shear structures into a highly sheared largerscale field can destabilize the field (e.g. Kusano et al., 2012; Muhamad et al., 2017). Reconnection between these small magnetic perturbations and the pre-existing sheared loops can result in flux-rope eruption and large-scale flaring.

Resolving these small-scale precursors is important for CME modeling. It has been known for some time that, at least in some cases, the onset of a coronal mass ejection can precede flaring by several minutes, with the CME itself coinciding with precursor indicators (e.g. Harrison et al., 1985; Harrison and Sime, 1989). This suggests that some precursors may serve as early signatures of the erupting fields. Current CME models (Toriumi and 


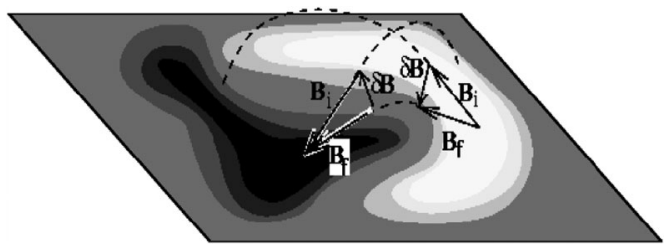

Figure 6 Photospheric magnetic-field changes associated with a flare. The initial photospheric-field vectors $\boldsymbol{B}_{i}$, the change in magnetic field $\delta \boldsymbol{B}$, indicating coronal restructuring during the flare/CME, and final state $\boldsymbol{B}_{f}$. The associated change in the connectivity of the coronal field is represented by the dashed lines. The changes agree with the expectation that the photospheric field should become more horizontal. Adapted from Hudson, Fisher, and Welsch (2008).

Wang, 2019) can be grouped into two broad classes: flux-rope models (Low, 1996; Lin and Forbes, 2000; Fan and Gibson, 2007), which assume that a flux rope exists before the eruption onset (Figure 5), in which case the precursors reflect the flux rope's sudden rise, and sheared magnetic-arcade models (van Ballegooijen and Martens, 1989; Mikic and Linker, 1994; Antiochos, DeVore, and Klimchuk, 1999; Longcope and Beveridge, 2007; Karpen, Antiochos, and DeVore, 2012; Doyle et al., 2019), which postulate that the flux rope is formed during the initial stages of the flare, implying that precursor signatures are the manifestation of the flux-rope formation process itself. Not all models embrace this dichotomy, for example the flux-cancelation model (e.g. Amari et al., 2010) involves elements of both. Careful DKIST studies of small-scale precursors are critical to discriminating between these possibilities.

\subsection{Changes in Magnetic Field Associated with Flares and CMEs}

What are the differences between the pre- and post-flare/CME magnetic-field configurations? How do the magnetic-field changes depend on height and time? What are the theoretical and practical implications of these changes? For example, how are flare emission and $C M E$ energy related to the magnetic restructuring?

Flares and coronal mass ejections are one of the most spectacular manifestations of solar activity (e.g. Webb and Howard, 2012). Flares occur when stressed magnetic fields in the low corona abruptly reconfigure, accessing a lower energy state. The rapid conversion of magnetic energy into plasma heating and bulk motion causes abrupt increase in the coronal and chromospheric active-region emission over a wide range of wavelengths. The field reconfiguration also propagates downward into the lower and denser atmospheric, with resulting changes in the photospheric magnetic field (Figure 6). This recnfiguration can be either transient or longer lived (Sudol and Harvey, 2005; Wang and Liu, 2015). Flares are often accompanied by CMEs and the onset of CMEs has been associated with flaring, although flares without CMEs and CMEs without flaring also occur (e.g. Kawabata et al., 2018, and the references therein).

Direct measurement of the pre-flare/pre-CME magnetic field and the post-flare/postCME reconfigured field has both conceptual and practical importance, contributing to our understanding of the underlying reconnection processes and flare-precursor configurations (e.g. Kontogiannis et al., 2019; Korsós et al., 2020a,b). A significant open question is whether precursor signatures, such as those based on the magnetic-field helicity or gradient for example, are robust and reliable over a range flare of energies. The anticipated 
spatial resolution of DKIST measurements will allow fine-scale determination of these precursor properties (Section 4.1). Beyond their contribution to the possible improvement of flare and CME initiation predictions, such measurements of the underlying magnetic field play a critical role in assessing CME geo-effectiveness. While all CMEs are large plasmacontaining magnetic structures expelled from the Sun, they exhibit a variety of forms. Some have the classical "three-part" structure: a bright front, interpreted as compressed plasma ahead of a flux rope, along with a dark-cavity and a bright compact core (Riley et al., 2008, and the references therein). Others display a more complex geometry or can appear as narrow jets (Webb and Howard, 2012). These forms reflect differences in internal magnetic morphologies, which subsequently determine interplanetary effects, including the formation of interplanetary shocks (an important source of solar energetic particles) and initiation of transient disturbances at Earth (upon interaction with the Earth's magnetosphere): both components of space weather.

Direct measurement of the pre- and post-flare/CME magnetic-field morphology is very challenging. The magnetic-field changes often occur on very short time scales, and they propagate quickly through the layers of the solar atmosphere (e.g. Wang and Liu, 2015; Wang et al., 2017a; Petrie, 2019). Outside of the photosphere, low photon counts make it hard to achieve the needed sensitivity at the required cadence, and multi-wavelength fullStokes studies are consequently difficult (Kleint, 2017). It is such studies that are, however, key to quantitative knowledge of flare and CME magnetism. While existing white-light CME observations provide information on the mass content of the CME and $\mathrm{x}$-ray and EUV observations provide diagnostics of the flare and CME plasma thermal properties, regular measurements of the magnetic-field strength and orientation, at multiple heights and extending into the corona, are essential to the further development and validation of eruption models. These require DKIST capabilities.

Importantly, DKIST on-disk, limb, and off-limb capabilities will allow quantitative assessment of the relationship between plasma-sheet observations and reconnection currentsheet properties. Current sheets are an important component of most solar-flare models. They are an essential element of post-CME flaring, and thin off-limb high-density hightemperature plasma structures, suggestive of current sheets, are observed above limb flares following eruptions (e.g. Savage et al., 2010; Liu, 2013; Seaton, Bartz, and Darnel, 2017). Spectroscopic observations have led to detailed understanding of the plasma properties within these structures ( $\mathrm{Li}$ et al., 2018; Warren et al., 2018), with implications for the underlying reconnection and field-reconfiguration processes (Longcope et al., 2018). Direct measurements, using DKIST instrumentation, of the magnetic field in and around these plasma sheets will allow more accurate assessments of the amount of flux opened by an eruption, better understanding of the evolution of the post-eruption field, and clarification of the mechanisms underlying the slow (sub-Alfvénic) outflows observed during flarereconnection events (e.g. Wang et al., 2017b, and the references therein).

The DKIST instrument suite will enable regular multi-wavelength spectropolarimetric measurements at high temporal cadence and spatial resolution in the solar photosphere, chromosphere and low corona. These will allow assessment of the local magnetic-field properties, and determination of flare- and CME-induced changes in that field, simultaneously over several heights in the solar atmosphere. When coupled to x-ray and EUV/UV observations from available and future space observatories, DKIST will, for the first time, allow us to directly connect coronal magnetic-field evolution to flare and CME emission and plasma properties. It will enable the careful measurement of the magnetic-field reconfiguration that occurs at the origin of space-weather events. 

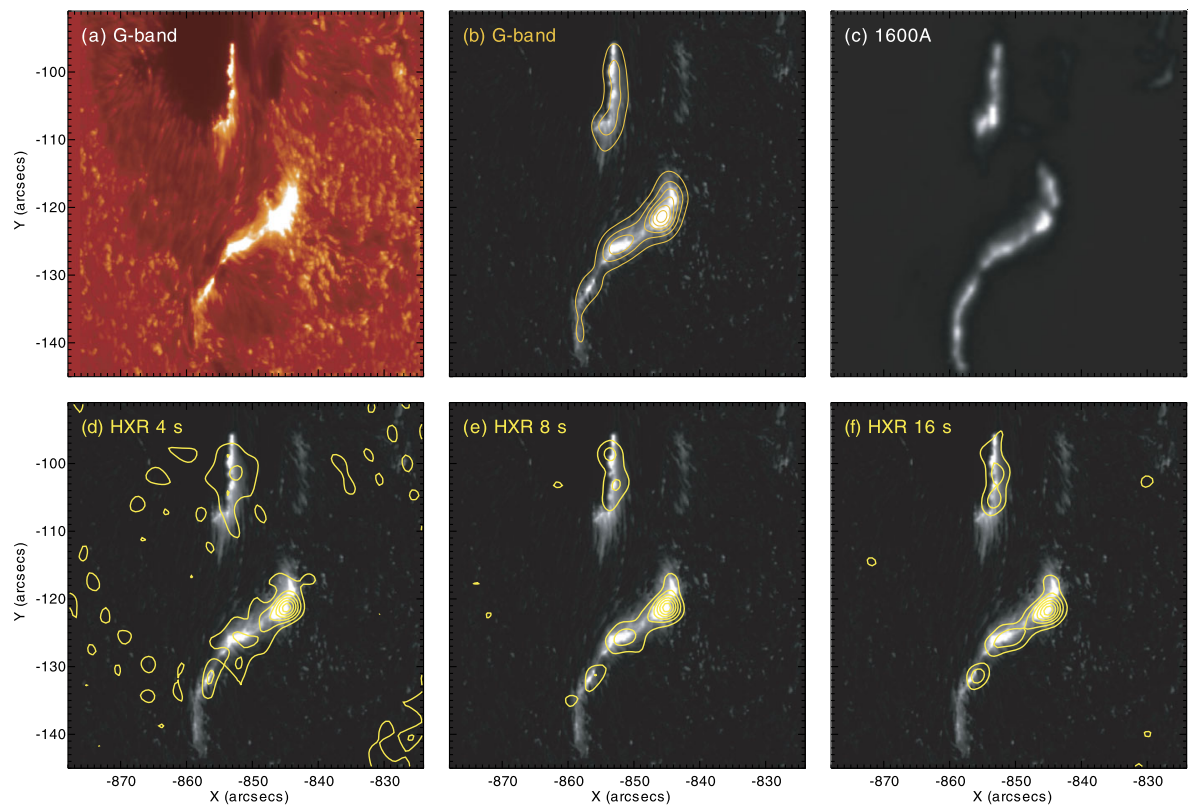

Figure 7 Optical flare ribbons at the time of the hard X-ray peak (X6.5, S06E63, SOL2006-1206T18:43:38.5). (a) Hinode/SOT G-band, (b) the same image as (a) after pre-flare subtraction, (c) TRACE $160 \mathrm{~nm}$ image taken nearly simultaneously, and (d) through (f) RHESSI contours (levels 15, 30, 45, 60, 75, and 90 percent of the peak flux) in the $25-100 \mathrm{keV}$ range for 4-, 8-, and 16-second temporal integrations, respectively. From Krucker et al. (2011), copyright by AAS.

\subsection{Energy Deposition During Flares}

Waves or beams? How is flare energy transported from the corona to the chromosphere? Where are the non-thermal particles produced? What is the origin of the flare optical continuum? How compact are flare kernels? What explains the large widths of chromospheric flare emission lines?

Flare energy, liberated in the corona, is transported to and dissipated in the chromosphere and upper photosphere, resulting in localized heating and intense bursts of radiation across the electromagnetic spectrum. This leads to chromospheric expansion and drives mass motions, upward-moving chromospheric "evaporation", and downward-flowing "condensation". In some locations, hard X-ray (HXR) bremsstrahlung emission is observed, revealing the presence of non-thermal electrons containing a significant fraction of the total flare energy. Ultimately, the majority of a solar flare's radiated energy is emitted in near-UV and optical lines and continua (e.g. Woods et al., 2004), and these provide rich diagnostics of the energy transport and deposition processes (Figure 7).

One of the longest-standing and most-important questions in flare physics is the origin of the optical continuum, which was first observed during the famous Carrington-Hodgson flare of 1859 and generally accounts for a large fraction of the total flare emission (Neidig, 1989). There are two primary candidates (e.g. Heinzel et al., 2017): the optical component of an enhanced black body (photospheric $\mathrm{H}^{-}$continuum enhancement) or hydrogenrecombination continuum (Paschen and Balmer). It is difficult to disentangle these two contributions (Kerr and Fletcher, 2014), but doing so would allow flare-energy transport 
and deposition-height discrimination since the former implies penetration of a significant amount of energy down into the photosphere while the latter carries information about evolution of the ionization state higher up in the chromosphere. Understanding the relative contribution of these would significantly constrain the transport mechanisms. Moreover, as the continuum emission carries away a large fraction of the flare energy, making it unavailable to drive a dynamical response, determining its origin is relevant to understanding why some flares are important acoustic sources while others are not (Lindsey et al., 2014).

High-temporal-cadence high-spatial-resolution observations will help to distinguish these processes (Hudson, Wolfson, and Metcalf, 2006; Xu et al., 2006; Fletcher et al., 2007; Martínez Oliveros et al., 2011, 2012; Kleint et al., 2016; Yurchyshyn et al., 2017). The standard flare model suggests that flare energy is transported from the corona to the chromosphere by beams of non-thermal electrons. If this is correct, the only way to significantly heat the photosphere is by reprocessing the electron-beam energy via back-warming by Balmer and Paschen continuum radiation originating in the mid to high chromosphere (Allred et al., 2005). Radiation-hydrodynamical models can predict the light curves of Balmer and Paschen continua consistent with the temporal evolution of the electron-beam energy deposition (Heinzel and Kleint, 2014; Kowalski et al., 2015). Of particular importance are observations near the Paschen limit. While the Balmer limit is not observable with the DKIST, the Paschen limit $(820.5 \mathrm{~nm}$ ) is, and, as with the Balmer limit (Kowalski et al., 2017a), observations of Paschen line broadening near the Paschen limit and the presence or absence of a continuum jump there (Neidig and Wiborg, 1984) can constrain the charge density and optical depth of the flaring solar chromosphere (Kowalski et al., 2015). Achieving high spatial resolution and spectral resolution is critical because unequivocal evidence for small, dense chromospheric condensations during the impulsive phase of solar flares has been established (Kowalski et al., 2017b), and these sites of intense localized heating are likely key to the interpretation of flare spectra.

While the standard flare model invokes energy transported from the corona to the chromosphere by beams of non-thermal electrons, the dominant energy transport mechanism is still under debate. Chromospheric heating to megaKelvin temperatures can start before observable HXR emission begins (Fletcher et al., 2013), and the apparent low height of HXR and optical sources during limb flares appears inconsistent with the expected penetration depths of accelerated electrons (Hudson, Wolfson, and Metcalf, 2006; Krucker et al., 2010; Martínez Oliveros et al., 2012). Moreover, the electron-beam densities implied by the HXR intensities observed suggest that a very large fraction of the pre-flare coronal thermalelectron population is accelerated (Krucker et al., 2010; Fletcher et al., 2013; Krucker and Battaglia, 2014), so it is unclear whether the electron-beam requirements can be met at coronal densities. Finally, issues involving the origin, amplitude, and stability of the return currents required to support these dense coronal beams are not fully resolved (Alaoui and Holman, 2017). Together these considerations suggest that additional modes of energy transport may be required, as do very recent modeling results indicating that electron beams are incapable of producing coronal rain, which is often found shortly after the onset of loop flares (Reep, Antolin, and Bradshaw, 2020). Proposed additional transport mechanisms include ion beams (Hurford et al., 2006; Zharkova and Zharkov, 2007), heat conduction (Longcope, 2014; Graham, Fletcher, and Labrosse, 2015; Longcope, Qiu, and Brewer, 2016), and Alfvén waves (Russell and Fletcher, 2013; Reep and Russell, 2016; Reep et al., 2018). Each results in different energy-deposition rates as a function of height in the solar chromosphere, influencing the local atmospheric properties (e.g. Kerr et al., 2016) such as temperature, density, bulk and turbulent velocities, ionization fractions, and atomic-level populations as a function of time, and thus in turn each has a potentially unique spectral evolution signatures. 
Figure 8 Image of a flare ribbon taken with the $1.6 \mathrm{~m}$ Goode Solar Telescope's Visible Imaging Spectrometer (VIS), $+0.1 \mathrm{~nm}$ off-band the $\mathrm{H} \alpha 656.3 \mathrm{~nm}$ line center (0.007 $\mathrm{nm}$ band pass), showing the ribbon crossing sunspots, coronal rain in the post-flare loops, and fine-scale brightenings at the foot points of the falling plasma in the chromosphere. From Jing et al. (2016).

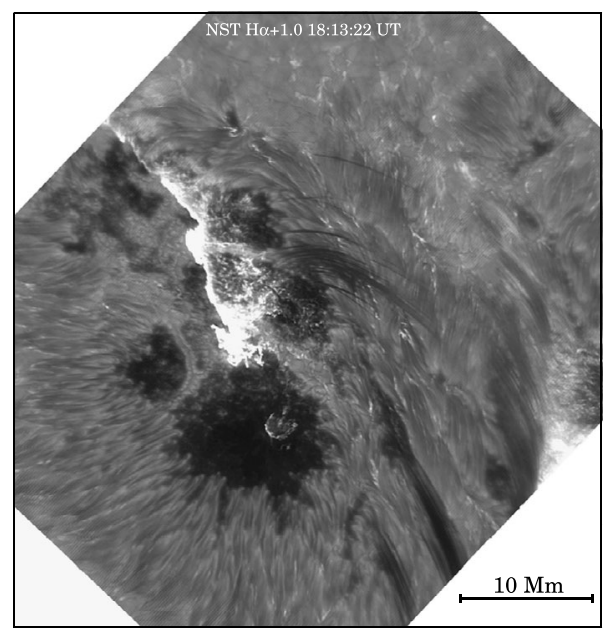

Multi-height DKIST observations, hand-in-hand with advanced radiation hydrodynamics simulations, will be critical to using those signatures to differentiate between the proposed energy transport and deposition mechanisms.

\subsection{The Fundamental Structure and Evolution of Flare Ribbons}

What is the mapping between the evolution of a flare ribbon and the reconnection process? What does flare-ribbon evolution tell us about changes in the overlying three-dimensional field and the particle-acceleration and heating processes?

Flare ribbons are localized, elongated brightenings in the chromosphere and photosphere. They are the sites of energy deposition at the foot points of the magnetic loops involved in flare reconnection. The brightening, due to the increased temperature of the flaring chromospheric plasma, is believed to be primarily due to collisional heating by flare-accelerated non-thermal electrons, although questions about the viability of this mechanism remain (Section 4.3 above). The brightest parts of a flare ribbon can be subject to localized heating of $10^{12} \mathrm{ergs} \mathrm{cm}^{-2} \mathrm{~s}^{-1}$ (Krucker et al., 2011), on the order of 100,000 times the energy flux required to heat the quiet solar chromosphere. The detailed structure and evolution of flare ribbons can provide insight into the fundamental length and time scales of flare-energy deposition and the link between particle acceleration and the evolving coronal magnetic field.

Flare ribbons occur in pairs that show a characteristic temporal evolution, reflecting changes in the overlying magnetic field. Conjugate ribbons separate in time, mapping out the progress of coronal magnetic reconnection. When observed simultaneously with the photospheric or chromospheric magnetic field, the motions of ribbons as a whole, and of the individual sources composing them, can be used to obtain the coronal magnetic-reconnection rate (Isobe et al., 2002; Qiu et al., 2002; Fletcher, 2009; Liu et al., 2018). Distinct brightenings are often observed to run along the ribbons, and have been linked to properties of threedimensional "slip-running" reconnection (Dudík et al., 2014). Moreover, since the evolution of the flare ribbon reflects changes in the large-scale coronal magnetic field, careful observations can inform our understanding of the dynamics of eruptive (CME) events (e.g. Sun et al., 2017; Hinterreiter et al., 2018). 
At small scales, flare ribbons show significant substructure (Figure 8), which on arcsecond scales is well aligned with regions of high vertical photospheric current density (Janvier et al., 2014). These numerous small-scale emission sources appear differently at different wavelengths, reflecting structured energy deposition. In line cores, flare ribbons appear stranded, as if a small portion of numerous magnetic loop tops in the chromosphere are illuminated (e.g. Mikuła et al., 2017). In the visible and infrared continua or in the far wings of spectral lines, individual sources are very compact with clear evidence for sub-arcsecond $(100 \mathrm{~km})$ structure, suggesting very small scales for individual flaring loops or current dissipation sites (Sharykin and Kosovichev, 2014; Jing et al., 2016). The sizes of the sources vary with wavelength, and this has been interpreted as being due to the narrowing with depth of the chromospheric magnetic-flux tube along which energy is being deposited (Xu et al., 2012). Additionally, individual source sites display a "core-halo" continuum-intensity substructure (Neidig et al., 1993; Hudson, Wolfson, and Metcalf, 2006; Xu et al., 2006; Isobe et al., 2007). This may be a signature of spatially varying heating across the magnetic structures or of two heating components: direct heating by energetic particles in the core and indirect heating by radiative back-warming in the halo (Neidig et al., 1993; Xu et al., 2006).

Flare kernels are the very brightest of these sources in flare ribbons, and they are the locations most clearly associated with the strong hard X-ray emission generated by nonthermal electrons. In the very earliest stages of a flare, flare kernels can expand significantly on timescales less than one second (Xu et al., 2010), possibly as a result of reconnection spreading through a highly stressed region of the coronal magnetic field. As discussed above, flare ribbons themselves also spatially propagate as the site of reconnection changes. At the leading edge of a propagating ribbon, sites of particularly large Doppler line broadening are observed (Jing et al., 2016; Panos et al., 2018), suggesting that the strongest energy input into the lower chromosphere and photosphere occurs at the locations of the most recently reconnected field. DKIST's aperture and adaptive-optics capabilities will enable regular subarcsecond measurements of the underlying spatial and temporal correlations, the heightdependent flows, and the evolving kernel substructure, to determine the flare energy flux into the solar chromosphere and the fundamental scales at which discrete coronal reconnection occurs (Xu et al., 2012; Graham and Cauzzi, 2015).

Moreover, the DKIST-VISP spectral bandpass will be wide enough to measure the exceptionally strong shock fronts that can accompany large flares. The magnitude of the observed Doppler red shifts in current observations of C-class solar flare ribbons (such as those obtained with CRisp Imaging Spectro-Polarimeter (Scharmer et al., 2008) on the $1 \mathrm{~m}$ Swedish Solar Telescope (Scharmer et al., 2003)) can exceed $0.1 \mathrm{~nm}$ relative to the $\mathrm{H} \alpha$ line center (Druett et al., 2017). These large red shifts are attributed to the development of a shock fronts in the upper chromosphere that propagate downwards towards the photosphere with velocities up to $50 \mathrm{~km} \mathrm{~s}^{-1}$ soon after flare onset and electron-beam injection. Supporting models of $\mathrm{H} \alpha$ line formation indicate that stronger flares yield even stronger shock fronts with even greater Doppler red shifts, up to $0.4 \mathrm{~nm}$, not yet observed with modern instruments. DKIST's ability to capture these larger red shifts within the VISP spectral band pass will contribute significantly to a detailed understanding of solar-flare electron-beam properties and how they scale with flare energy.

\section{Magnetic Connectivity Through the Non-eruptive Solar Atmosphere}

The solar magnetic field extends from the solar interior, across the photosphere, through the chromosphere and transition region, and into the corona, permeating the entire volume and 
out into the heliosphere. Plasma motions in the solar convective envelope produce a highly structured photospheric magnetic-field distribution, which can be considered the boundary condition for the magnetic field above. As the photospheric boundary field evolves, the field above must reconfigure, and, because the atmosphere extends over many scale heights, the energetics and dynamics of the response are diverse and multi-scaled. A central goal of the DKIST science mission is a quantitative understanding of the complex interplay between flows, radiation, heat conduction, wave propagation and dissipation, and reconnection in the solar atmosphere. This understanding is critical to the resolution of long-standing problems in solar and stellar astrophysics such as chromospheric and coronal heating, the origin and acceleration of the solar wind, and the propagation of magnetic disturbances into the heliosphere.

Understanding the complex, connected solar atmosphere, with its widely varying ionized and partially ionized plasma regimes, requires diverse, flexible, and multi-spectral-line polarimetric instrumentation capable of simultaneously probing many heights at high temporal cadence and over small spatial length scales. DKIST is at the forefront of these observational challenges. Its new capabilities in high-precision spectropolarimetry will allow the inference of magnetic-field properties with spatial and temporal resolutions never before achieved. Those inferences will depend on advanced and challenging inversions. For example, while the distribution of solar photospheric magnetic fields has been routinely measured since the invention of the Zeeman-effect-based magnetograph, measurement of the chromospheric field and its connections to the upper solar atmosphere requires interpretation of spectral lines formed under conditions of non-local thermodynamic equilibrium (NLTE) in which both Zeeman and atomic-level polarization processes are important. DKIST aims to facilitate these with chromospheric data of unprecedented precision.

DKIST is also at the forefront of coronal spectropolarimetry. Extrapolation of the magnetic field into the solar corona is inherently limited by assumptions about the distribution of currents in the magnetized volume. To advance our understanding of, for example, the available free magnetic energy driving solar flares and eruptions, remote sensing of the magnetic field in the corona itself is necessary. DKIST, as the world's largest coronagraphic polarimeter, will allow the measurement of the full Stokes spectra of the forbidden magnetic-dipole emission lines of highly ionized metals in the corona. These can be used to determine the topology and evolution of the coronal field and, along with DKIST chromospheric diagnostics, its connectivity to the lower atmosphere.

Several critical science topics in this research area are discussed in detail below, including i) the mass and energy cycle in the low solar atmosphere, ii) the origin and acceleration of the solar wind, iii) magnetic reconnection throughout the solar atmosphere, iv) waves in the solar atmosphere, v) impact of flux emergence on the non-eruptive solar atmosphere, vi) multilayer magnetometry, and vii) large-scale magnetic topology, helicity, and structures.

\subsection{Mass and Energy Cycle in Low Solar Atmosphere}

How important are spicules and other jet-like phenomena to the chromosphere-corona mass cycle? What is the role of spicule heating in the coronal energy balance? Can we characterize and model the coronal-rain phenomenon well enough to understand its role as a return flow?

All coronal plasma has its origins in the lower solar atmosphere, with the coronal mass budget determined by a balance between upward flows (evaporative or eruptive), downward mass transport (e.g. coronal rain), and solar-wind losses. One of the key outstanding challenges to furthering our understanding of the solar atmosphere is determining which of many 


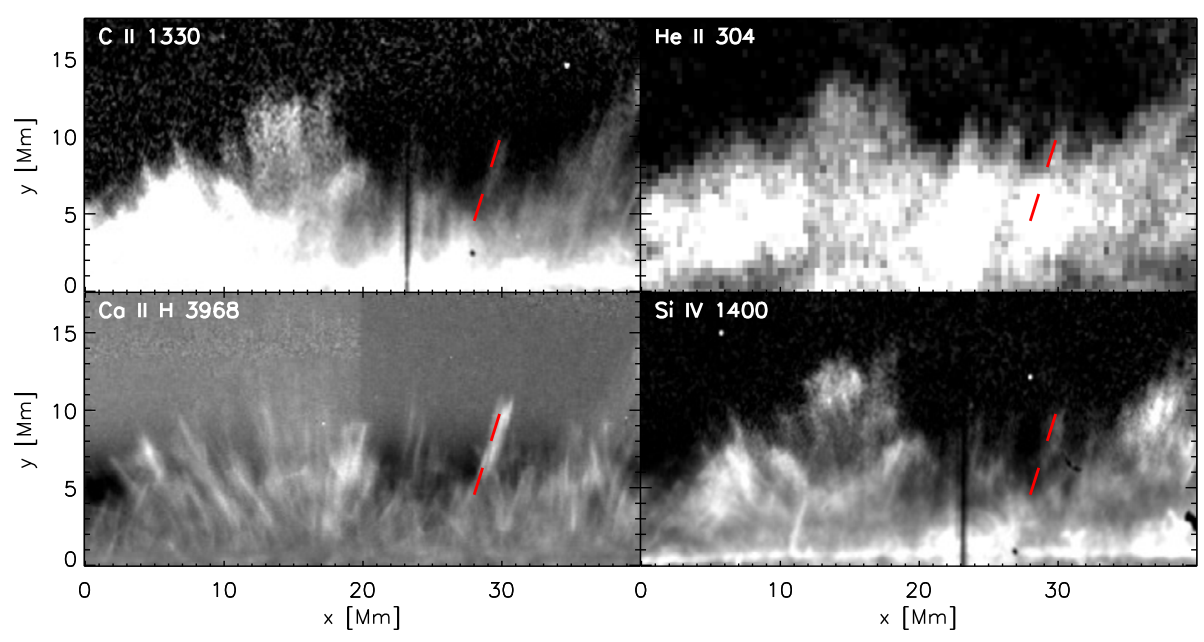

Figure 9 Simultaneous images of spicules in Ca II H $396.8 \mathrm{~nm}$ (Hinode), C II $133.0 \mathrm{~nm}$ and Si IV $140.0 \mathrm{~nm}$ (IRIS), and He II $30.4 \mathrm{~nm}$ (SDO/AIA). Future coordinated observations with DKIST, ALMA, and other observatories are key to addressing unresolved questions about spicule heating and their role in the mass and energy balance of the transition region and corona. Image from Skogsrud et al. (2015). Reproduced with permission; copyright 2015 American Astronomical Society.

process that appear to be involved dominates the transfer of mass between the cool chromosphere and hot corona in regions of differing magnetic topology. Previous observations have provided a partial view of the mass cycle, but the small spatial and temporal scales involved have significantly hindered advancement. DKIST will provide a more complete view, especially when combined with coordinated observations using space-based observatories, such as IRIS, Hinode, and Solar Orbiter, or the ground-based radio telescope array ALMA (Atacama Large Millimeter/sub-millimeter Array), which allows complementary temperature and soon polarization diagnostics of the solar chromosphere (see Yokoyama et al., 2018).

Jets play an important role in the chromosphere-corona mass cycle. They are observed to have a wide range of spatial scales, from spicules with widths of a few hundred kilometers, to larger chromospheric jets with widths of $\approx 1000 \mathrm{~km}$, to coronal jets with widths of a few thousand kilometers. Recent observations show that some jets are driven by the eruption of a small-scale flux ropes (Sterling et al., 2015) triggered by flux cancelation at the magnetic neutral line (Panesar et al., 2016, 2018; Panesar, Sterling, and Moore, 2017), but the full range of magnetic-field reconfiguration scenarios that lead to the jets observed is not known. DKIST's spectropolarimetric capabilities will significantly contribute to our understanding of jet initiation, allowing us to better quantify how effectively each jet type injects plasma into the solar atmosphere.

Spicules appear to be the most ubiquitous jet-like feature in the solar chromosphere (Figure 9). They are highly dynamic, vary on time scales of 10-30 seconds, and are finely structured, with widths $<300 \mathrm{~km}$ and substructure at scales below that. Spicules transport plasma upwards into the solar atmosphere at speeds of $10-200 \mathrm{~km} \mathrm{~s}^{-1}$ and may, either alone (De Pontieu et al., 2011) or in combination with other slower jets (e.g. Morton et al., 2012), play a significant role in the mass and energy balance of the corona and solar wind. Yet, despite having been observed over a wide range of wavelengths from EUV to visible, we do not understand the mechanisms responsible for spicule formation, how they are heated, often to transition-region temperatures or higher (Pereira et al., 2014; Henriques et al., 2016; 
Samanta et al., 2019), the role that hydromagnetic waves play in their dynamic evolution, or their full impact on the mass and energy balance of the outer solar atmosphere (Pereira, 2019). That coronal signatures of spicules have been observed at the smallest scales above both active regions (De Pontieu et al., 2011) and the quiet Sun (Henriques et al., 2016) suggests that they may play a global role as heat and mass conduits between the chromosphere and corona, but our understanding of their importance is limited by current instrumentation capabilities. Spicule-induced mass transport to coronal heights is estimated by some authors to be two orders of magnitude larger than the solar-wind mass flux (Beckers, 1968; Sterling, 2000), but others suggest that the role of spicules in the mass cycle is rather limited compared to that of more uniform chromospheric evaporation due to small-scale flaring processes (e.g. Klimchuk, 2012). In fact, there is still some debate about the fundamental nature of spicules, whether only one or multiple spicule types exist (De Pontieu et al., 2007a; Zhang et al., 2012, but see Pereira, De Pontieu, and Carlsson, 2012), or whether they are indeed jets of accelerated plasma, with an alternative suggestion that the apparent upward motions are due instead to line-of-sight integration through evolving warped two-dimensional magnetic sheet-like structures (Judge, Tritschler, and Low, 2011; Judge, Reardon, and Cauzzi, 2012; Lipartito et al., 2014).

DKIST observations, employing a wide range of chromospheric spectral lines, will provide revolutionary new views of the fine-scale structure, magnetic and electric fields (Anan, Casini, and Ichimoto, 2014) within, and thermodynamic evolution of, spicules and other chromospheric jets. DKIST will be able to capture the dynamic evolution of jets at high cadence ( $\lesssim 3$ seconds) and make simultaneous measurements (with $\lesssim 15$-second cadence) of the chromospheric and photospheric magnetic fields and flows that underlie jet initiation and acceleration. Together these will help clarify issues such as the relationship between jet initiation and the apparently ubiquitous Alfvén pulses that are excited by swirling motions in the solar photosphere (Goodman, 2012; Liu et al., 2019a,b) and the roles and relative importances of processes such as reconnection (Section 5.3 below), magnetic tension amplification by ion-neutral coupling (Martínez-Sykora et al., 2017), micro-filament eruption (Sterling and Moore, 2016), and other small-scale processes.

As already indicated, some have suggested that, while jet-like features are perhaps the most prominent, they may not be the most important component of the chromospherecorona mass cycle, that more gentle processes play an important, even dominant, role. One sensitive signature of mass transport, momentum flux, and wave heating is the degree of elemental fractionation, i.e. the degree to which elements of low (lower than about $10 \mathrm{eV}$ ) first ionization potential (FIP) are enriched or depleted compared to other elements (the FIP or inverse-FIP effects: Meyer, 1985a,b; Feldman, 1992; Feldman and Laming, 2000; Laming, 2015). Because FIP fractionation is sensitive to the thermodynamic and electromagnetic plasma environment, it depends on the atmospheric height of the region being considered, the magnetic-field geometry (open or closed) and connectivity, the heating mechanisms at play, the bulk plasma flow speed, and the dominant magnetohydrodynamic (MHD) wave modes present (Laming, 2015). In fact, spicules themselves may be responsible for the absence of the FIP effect at temperatures below $10^{6} \mathrm{~K}$ on the Sun (Laming, 2015, and the references therein). These sensitivities, when understood well, allow FIP fractionation measurements to be used to constrain the solar-wind source region and thus the origin of plasma sampled during in-situ heliospheric measurements (e.g. Geiss, Gloeckler, and von Steiger, 1995; Parenti et al., 2000; Brooks and Warren, 2011; Brooks, Ugarte-Urra, and Warren, 2015; Baker et al., 2015). Coordinated observations (Section 6.5), combining chromospheric measurements by DKIST with in-situ measurements of the heliospheric plasma properties by Solar Orbiter and Parker Solar Probe, will allow unprecedented identification and characterization of the sources of the fast and slow solar wind. 
Another key aspect of the chromosphere-corona mass cycle is the return flow from the corona to the lower atmosphere. One component of this is prominently visible at the solar limb as coronal rain: a finely structured and multi-thermal flow that appears to be driven by cooling instabilities (e.g. Antolin and Rouppe van der Voort, 2012; Antolin et al., 2015b; Mason, Antiochos, and Viall, 2019, and the references therein). The descending material is visible in the off-limb active corona at transition-region and chromospheric temperatures, and can be observed in what are typically chromospheric optical spectral lines (Kawaguchi, 1970; Schrijver, 2001; Antolin, Shibata, and Vissers, 2010). Current observations are unable to fully resolve coronal rain: the peak of the coronal-rain element-width distribution remains unresolved (Scullion et al., 2014). Thus we do not yet have a full accounting of the total rain drainage rate. Measurements of loop oscillations can be used to determine the thermally unstable mass fraction in a coronal-loop system and thus provide further constraint on that rate. Since coronal-rain condensations are coupled to the magnetic-field lines they track the oscillatory motion of the loops (Kohutova and Verwichte, 2016) and the evolution of the oscillations can be used to deduce the fraction of the loop plasma mass that becomes thermally unstable and drains with time. This seismic estimation provides more than a consistency check on the drainage rate because it also allows measurement of the unresolved coronal-rain mass fraction (Froment et al., 2018), which in turn constrains the fundamental spatial scales of the rain and the fine-scale structure of coronal loops. DKIST observations will push these inferences even further.

One-dimensional hydrodynamic loop models (Mendoza-Briceño, Erdélyi, and Sigalotti, 2002; Müller, Hansteen, and Peter, 2003; Mendoza-Briceño, Sigalotti, and Erdélyi, 2005) produce catastrophic cooling events that generate intermittent and repeating rain-like downflows even when steady, exponentially decaying with height, foot-point concentrated heating is employed. Similarly, 2.5-dimensional and recent three-dimensional simulations form fine-scaled localized rain elements even when a spatially smooth but localized foot-point concentrated heating function is applied (Fang, Xia, and Keppens, 2013; Moschou et al., 2015). These numerical studies, along with observational evidence for foot-point concentrated heating in active regions (Aschwanden, 2001), suggest that studies of coronal rain are important, not just in the context of the mass cycle, but also in constraining heating mechanisms. The statistical properties of the rain depend on the spatial distribution of the heating because the heating location influences the thermal stability of the plasma in a coronal-loop system (Antolin, Shibata, and Vissers, 2010).

Observational contributions to our understanding of coronal rain depend on leveraging the high-resolution and multi-wavelength capabilities of DKIST to unravel its evolving, complex, multi-thermal behavior. With a mix of hot (ionized) and cold (neutral) gas, coronal rain is an ideal context within which to study ion-neutral interaction effects, such as ambipolar diffusion, that are expected to play key roles more broadly in the dynamics of the partially ionized chromosphere. But the rain's thermal state is complex and difficult to characterize. Hydrogen is likely out of ionization equilibrium and other elemental ionization ratios are poorly constrained (Antolin, Shibata, and Vissers, 2010; Antolin et al., 2015b). DKIST will also be able to provide spectropolarimetric measurements from which the vector magnetic field within rain-producing loop systems can be inferred. Such inferences are fundamental in characterizing the underlying flow instabilities (e.g. Martínez-Gómez et al., 2020), but they will require careful theoretical underpinning. Recent studies have been able to exploit the Zeeman effect in the Ca II $854.2 \mathrm{~nm}$ chromospheric line to assess the presence of strong fields $(100-300 \mathrm{G})$ in bright post-flare loops systems (Kuridze et al., 2019), but this approach may be difficult in more quiescent cases, which are characterized by much weaker fields and lower photon counts. Significant progress may be possible if Hanle diagnostics in 
lines of neutral Helium such as the He I 1083 and $587.6 \mathrm{~nm}$ multiplets are employed (Schad, 2018), and polarimetric observations of these two He I multiplets with DKIST may provide the first view of sub-arcsecond scale weak magnetic fields (with sensitivity of a few Gauss) in quiescent loop systems.

\subsection{Coronal Heating, Solar Wind Origin and Acceleration}

Waves or nanoflares? What are the relative importances of different proposed coronalheating mechanisms? What are the solar-wind momentum sources? What role does the chromosphere play in coronal heating?

The steep transition from the cool chromosphere to the million-degree and higher corona is the direct result of the cooling catastrophe that results when hydrogen in the solar atmosphere becomes fully ionized so that radiative recombination can no longer cool the optically thin plasma (Woods, Holzer, and MacGregor, 1990a,b). The plasma temperature climbs high enough so that electron conduction back down to the chromosphere, and resulting radiative losses from there, are sufficient to balance the heating above. Solving the coronal-heating problem requires identifying the heat source, which in the statistically steady state balances thermal conduction to the chromosphere and any other smaller direct energy losses from the corona by radiation or advection. Significant progress has been made in the last decades, and it is now apparent that no single heating mechanism is likely universally dominant (e.g. Kuperus, Ionson, and Spicer, 1981; Zirker, 1993; Klimchuk, 2006; Erdélyi and Ballai, 2007; Parnell and De Moortel, 2012; De Moortel and Browning, 2015). The importance of mechanisms such as reconnection, MHD or plasma-wave dissipation, or turbulent dissipation likely vary depending on coronal conditions, particularly the magnetic-field configuration. Moreover, the mechanisms are highly intertwined and interdependent; dissipation of current sheets can produce waves and waves in magnetically structured media can induce current sheets (e.g. Velli et al., 2015), and observations indicate that even on very large scales eruptive flares can trigger oscillations in coronal loops and filament oscillations can induce eruption (e.g. Jess et al., 2015; Russell, Simões, and Fletcher, 2015, and the references therein) and associated flaring. Turbulence is similarly likely ubiquitous, and theoretical work suggests an important role for Alfvén-wave induced turbulence (e.g. van Ballegooijen et al., 2011; Asgari-Targhi et al., 2013; van der Holst et al., 2014).

Thus, while a number of general properties of coronal heating (e.g. De Moortel and Browning, 2015) have been observationally established (that coronal heating is unsteady and impulsive, that coronal magnetic fields store energy that can be dissipated via reconnection, that the corona supports a rich wave field, and that the corona can only be understood in conjunction with its coupling to the chromosphere) details are less certain (e.g. Klimchuk, 2015; Schmelz and Winebarger, 2015). Questions remaining include: What is the relative importance of different heating mechanisms? Do current sheets in the corona play an important role in heating, and if so what plasma processes are involved in their dissipation? What is the fundamental scale of the substructure in multi-thermal coronal loops, and what processes determine this? During small-scale reconnection events, how much energy is dissipated directly, how much is radiated as waves, and how much goes into acceleration of non-thermal electrons (Testa et al., 2014)? How does magnetic reconnection heat the plasma (Longcope and Tarr, 2015)? How and where are the ubiquitous MHD waves in the corona dissipated (e.g. Poedts, 2002; Gupta, 2017)? How far out in the solar wind does heating extend (Martinović, Klein, and Bourouaine, 2019, and the references therein)? What are the characteristic scales and magnitudes of the heating events? What triggers them? 

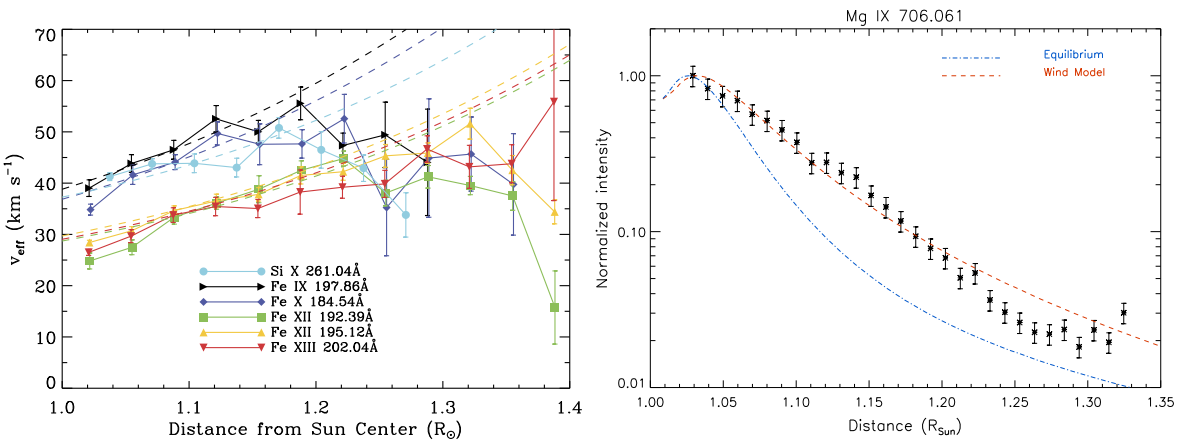

Figure 10 Hinode/EIS line-width measurements in an off-disk coronal hole as a function of height (left, from Hahn, Landi, and Savin, 2012). The decrease in width with distance off-limb beyond $1.1 \mathrm{R}_{\odot}$ suggests wave damping. Mg IX $70.6 \mathrm{~nm}$ off-disk line intensity in a polar coronal hole (right, from Landi et al., 2012). Measurements from SOHO/SUMER, with model estimates assuming ionization equilibrium shown in blue and those when employing non-equilibrium ion fractions in red. Image from Skogsrud et al. (2015), copyright by AAS (left, from Hahn, Landi, and Savin, 2012, copyright by AAS)/(right, from Landi et al., 2012, copyright by AAS).

DKIST will be fundamental in answering these questions because it will enable careful, repeated, and frequent measurements of the plasma properties of the inner corona, including the local magnetic-field strength and direction, at multiple heights. These will facilitate the quantitative evaluation of suggested heating processes in varying magnetic environments. Measurement of the line-continuum and line-line intensity ratios of ionic species, such as Fe IX to XV, that are also found in in-situ measurements of the fast and slow solar wind, will allow determination of the electron-density, temperature, and chargestate evolution of the solar-wind plasma, informing our understanding of the acceleration and heating processes (Figure 10, Landi et al., 2012; Landi, Habbal, and Tomczyk, 2016; Boe et al., 2018). Comparison between observations and theoretical studies of fast and slow magneto-acoustic wave-mode conversion, shock formation, and dissipation (e.g. Zhugzhda, Bromm, and Ulmschneider, 1995; Carlsson and Stein, 1997) will help constrain the role of these processes as a function of height in the chromosphere. For example, in models, high-frequency $(>10 \mathrm{mHz}$ ) propagating acoustic waves develop into radiatively damped weak shocks within the first few hundred kilometers above the photosphere (Carlsson and Stein, 2002) while lower-frequency waves $(\approx 4-10 \mathrm{mHz})$ develop into strong shocks in the chromosphere (above $1 \mathrm{Mm}$ ) where radiative damping is less effective (Priest, 2000). Multiheight DKIST observations will allow careful measurement of this frequency dependence in regions of differing magnetic-field configurations and thus an assessment of wave-energy deposition with height. Moreover, previous high-resolution observational work has reported evidence for shock-induced turbulence (Reardon et al., 2008), and such turbulence may provide a mechanism for the dispersal of the wave energy beyond the local shock region itself. Turbulence may also play a direct role in wave-mode conversion, coupling the compressive motions to Alfvénic fluctuations which can continue to propagate outward, transmitting energy to higher layers of the solar atmosphere. Alternatively, counter-propagating Alfvén waves may nonlinearly interact to produce MHD turbulence, dissipating the waves, and heating the plasma (van Ballegooijen et al., 2011). DKIST observations can differentiate between these processes in the solar atmosphere and determine their occurrence frequency.

Further, even though direct observation of individual nanoflare heating events lies beyond the capabilities of DKIST, DKIST observations will help distinguish between spe- 
cific reconnection heating mechanisms. Heating models based on flux cancelation have been previously motivated by high-resolution Imaging Magnetograph eXperiment (IMAX) data from Sunrise (Priest, Chitta, and Syntelis, 2018) and heating events in simulations of threedimensional kink-unstable flux ropes may be diagnosable using DKIST coronal lines (Snow et al., 2018). With DKIST, high-resolution chromospheric observations of intensity fluctuations and motions at the foot points of hot coronal loops should reveal key telltale signatures of nanoflares in the overlying corona (Testa et al., 2014). When coupled with radiativehydrodynamic modeling (e.g. Kerr et al., 2016; Polito et al., 2018), such observations may be able to constrain the properties of the non-thermal particles or waves generated at nanoflare sites. Finally, although the details are uncertain, it has been suggested that the chromosphere may play an important role in coronal heating (e.g. Withbroe and Noyes, 1977; Sturrock, 1999; De Pontieu et al., 2017) since chromospheric plasma can be heated to transitionregion temperatures and higher and be carried via jets to coronal heights. If so, the jet studies outlined in Section 5.1 are very relevant to this research topic as well.

The hot, outer solar corona escapes the Sun as the solar wind. The solar wind carries plasma out into the heliosphere, where it interactions with the planetary space environments and importantly influences CME arrival times and geo-effectiveness, yet there is no consensus understanding of where the solar wind originates or how it is accelerated. The fast solar wind and slow solar wind have different physical properties (e.g. Feldman, Landi, and Schwadron, 2005; Ebert et al., 2009), likely come from different source regions, and are possibly subject to different acceleration mechanisms. The fast wind originates in coronal holes, but how the detailed properties of the field and plasma within a coronal hole lead to the observed properties of the wind is not clear. For example, additional sources of momentum, beyond Parker's original gas-pressure gradient mechanism (Parker, 1958, 1963), are required for the plasma to reach the observed fast-wind speeds. Possible momentum sources included large-amplitude MHD waves (Alazraki and Couturier, 1971; Jacques, 1977; De Pontieu et al., 2007b; Thurgood, Morton, and McLaughlin, 2014), Type-II spicules (e.g. De Pontieu et al., 2009; Moore et al., 2011), resonant interactions with ion-cyclotron waves (Hollweg and Isenberg, 2002), and others (e.g. Cranmer and Winebarger, 2019, and the references therein), but these are to date poorly constrained by observations. Similarly, the slow wind has variously been proposed to originate from the edges of coronal holes, closed-open field boundaries within and bordering active regions, streamers (particularly streamer tops), or small coronal holes, but while these locations are all associated with magnetic reconnection between closed magnetic flux systems and open ones that connect to the wind, there is no consensus on the dominant underlying field configuration or acceleration mechanism (e.g. Feldman, Landi, and Schwadron, 2005; Cranmer, 2009). In fact, some of the observed differences between the fast and slow solar winds may have more to do with the expansion properties of the background magnetic fields along which they are streaming than with differences between their source regions (Wang and Sheeley, 2003).

The solar wind can be studied using either remote-sensing or in-situ techniques. Insitu measurements typically provide direct information on plasma properties only after the plasma has undergone much of its evolution, although Parker Solar Probe (PSP) is revolutionizing these measurements, aiming to sample, during its closest perihelia, regions of solar-wind heating and acceleration directly (e.g. Venzmer and Bothmer, 2018). Remotesensing observations, on the other hand, allow frequent multi-wavelength measurements of the solar-wind source regions, but can be difficult to interpret. Combining these types of measurements (Landi et al., 2012) is already yielding exciting results in the current earlyPSP era (Rouillard et al., 2020). DKIST will make significant contributions to these efforts (Section 6.5). Regular off-disk coronal measurements of the magnetic field and the plasma 
(a)

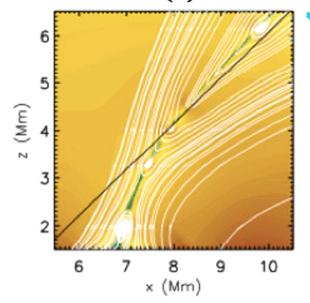

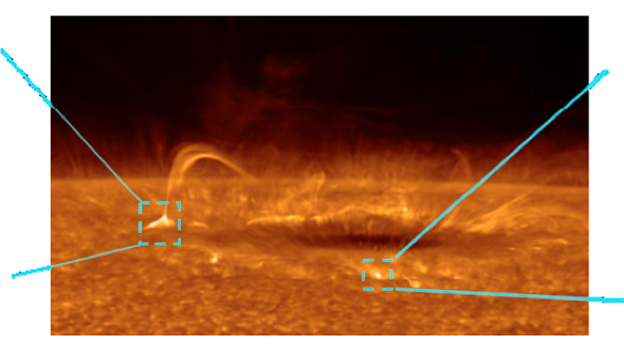

(b)

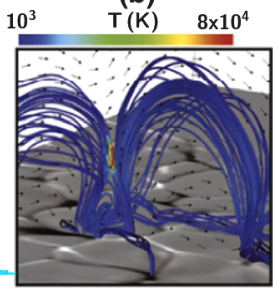

Figure 11 The chromosphere near a sunspot as observed with the Hinode/Solar Optical Telescope through the $\mathrm{Ca}$ II $\mathrm{H}$ broadband filter, showing transient brightenings, likely due to heating by reconnection events (middle, from Shimizu, 2015, fiducial boxes added). (a) Numerical simulation of a jet in which intermittent reconnection results from plasmoid instability. From Rouppe van der Voort et al. (2017). (b) Numerical simulation of an Ellerman bomb, in which reconnection occurs between emerging bipolar fields. From Hansteen et al. (2017). Reproduced with permission; copyright 2017 American Astronomical Society.

properties will characterize the solar wind as it emerges from its source up to a height of 0.5 solar radii above the limb. Since the visible and infrared spectral lines observed by DKIST largely result from photoexcitation, their intensities decrease more slowly with height (closer to being in proportion to the electron density rather than its square) than do those of EUV spectral lines, which result largely from collisional excitation (e.g. Landi, Habbal, and Tomczyk, 2016; Del Zanna and DeLuca, 2018). This makes plasma and magnetic-field diagnostics to the outer edge of the DKIST coronal field of view possible. Importantly, lower down, in the chromosphere and low transition region, where current space-based EUV observational efforts are focused, DKIST will enable inference of the vector magnetic field via their spectropolarimetric signatures in lines such as He I $1083.0 \mathrm{~nm}$.

DKIST's capabilities in combination with those of IRIS and Hinode will facilitate studies of solar-wind acceleration physics from the chromosphere through transition region and into the corona. Through coordinated efforts, these observatories will be able to address critical issues such as how the physical properties of the solar wind change with height, how that profile depends on position from the center to the edge of coronal holes, what defines coronal-hole-streamer boundaries, and how does reconnection at the boundary between closed and adjacent open field in active regions yield the observed wind properties. Plasma diagnostics will allow estimates of the mass and energy flow along magnetic-field lines, and coronal line-width studies will help in understanding wave propagation and damping (Hahn, Landi, and Savin, 2012). Combining magnetic-field, electron-density, and temperature measurements will make possible charge-state evolution modeling of the accelerating solar wind and aid in the development of empirical models of the solar-wind speed between 1 and $1.5 \mathrm{R}_{\odot}$ (Landi et al., 2012). Extrapolation to the freeze-in height, with field extrapolations no longer strictly dependent on the photospheric field, will provide further links to in-situ instrumentation on PSP, SO, and Advanced Composition Explorer (ACE) and constrain the solar-wind source locations (Section 6.5).

\subsection{Magnetic Reconnection in the Solar Atmosphere}

What is the three-dimensional geometry of the magnetic field near reconnection sites? What roles do ion-neutral collisions, current-sheet instabilities, and plasmoid ejection play? How efficiently does the reconnection heat and accelerate the solar plasma?

Magnetic reconnection is a fundamental process that transforms magnetic energy into kinetic and thermal energies in astrophysical plasmas. The magnetic energy is stored, main- 
tained, and amplified over extended periods of time (minutes to hours in magnetic network and hours to weeks in sunspots and active regions) before being released suddenly during reconnection events, with flares occurring on small spatial scales (kilometers) over very short times (minutes). Reconnection events can accelerate plasma in jet-like structures and induce local heating in the chromosphere and above.

In addition to the ubiquitous spicules (Section 5.1), jets are observed in sunspot penumbre (Katsukawa et al., 2007; Tiwari et al., 2018), at the edges of sunspots (Morton, 2012), in light bridges (Toriumi, Katsukawa, and Cheung, 2015; Tian et al., 2018), and in the plage regions surrounding sunspots (Nishizuka et al., 2011). These jets often display morphologies reminiscent of reconnection sites (e.g. Innes et al., 1997; Shibata et al., 2007; Singh et al., 2012), but there are few direct measurements of the local magnetic field and its reconfiguration by reconnection. Localized heating is also observed, for example as Ellerman bombs and UV bursts: point-like brightenings in the wing of chromospheric lines such as $\mathrm{H} \alpha$ and Ca II (e.g. Vissers et al., 2015; Reid et al., 2016; Rouppe van der Voort et al., 2017; Toriumi, Katsukawa, and Cheung, 2017; Young et al., 2018). These brightenings are often associated with bipolar moving magnetic features near sunspots or colliding bipolar structures in regions of emerging flux, similar to magnetic cancelation events in the quiet Sun (Rouppe van der Voort, Rutten, and Vissers, 2016; Nelson et al., 2017a). Reconnection is again implicated (Figure 11), but again without direct measurements of field reconfiguration. Further, brightening and excess line broadening is observed in transition region and coronal loops, consistent with impulsive reconnection heating, bidirectional flows, and ion-cyclotron turbulence at sites of magnetic braiding of the underlying multi-stranded field (Cirtain et al., 2013; De Pontieu et al., 2014b; Hansteen et al., 2014; Bahauddin, Bradshaw, and Winebarger, 2020).

An important goal of DKIST is to measure the magnetic-field changes associated with reconnection phenomena at high resolution and simultaneously over multiple heights in the solar atmosphere from the photosphere into the chromosphere. Diagnosis of the magnetic field at reconnection sites before and after reconnection events will facilitate reconstruction of the magnetic and thermodynamic history of the plasma in the reconnection volume. It will allow detailed assessment of the total field annihilation, reconnection rate, total magneticenergy release, and local heating induced. Together with recent in-situ measurements of the underlying microphysical processes in the Earth's magnetosphere (e.g. Burch et al., 2016; Torbert et al., 2018; Chen et al., 2020; Hesse and Cassak, 2020) and laboratory experiments (e.g. Dong et al., 2012; Gekelman et al., 2016; Olson et al., 2016; Howes, 2018; Takahata, Yanai, and Inomoto, 2019; Seo et al., 2020), such studies will advance our fundamental understanding of the energetics of solar and astrophysical reconnection, determining how the energy is partitioned between bulk flows and random motions, between plasma acceleration and heating (Ji and Daughton, 2011; Coates, 2016; Yamada, Yoo, and Myers, 2016).

A key aspect of DKIST's contributions to reconnection studies is the particular plasma environment that will be sampled. The lower solar atmosphere (photosphere and chromosphere) is relatively dense and weakly ionized, with an ionization fraction on the order of $10^{-4}$ at the height of the temperature minimum (e.g. Khomenko, 2017). Weakly ionized plasmas are found over a wide range of astrophysical settings, including the atmospheres of other cool stars, the warm neutral interstellar medium (ionization fraction of $10^{-2}$ : Jenkins, 2013), dense cores of molecular clouds (ionization fraction of $10^{-7}$ : Caselli et al., 1998), and protostellar and protoplanetary disks (ionization fraction $10^{-10}$ or less: e.g. Armitage, 2019). Importantly, low ionization fractions (in the range of those found in the solar chromosphere) increase reconnection rates. Ion-neutral interactions can lead to increased resistivity and an increase in the effective ion mass, with consequent reduction in the Alfvén speed, 
steepening of current sheets, heating of the inflow and exhaust regions of reconnection sites, and enhancement of the plasmoid instability (e.g. Zweibel, 1989; Chiueh, 1998; Zweibel et al., 2011; Leake et al., 2012; Mei et al., 2012; Murphy et al., 2012; Zweibel, 2015; Ni et al., 2020). These in turn have important implications for solar phenomena, likely being responsible for increased damping of MHD waves (De Pontieu, Martens, and Hudson, 2001), increased current dissipation and heating of the solar chromosphere (Khomenko and Collados, 2012; Martínez-Sykora, De Pontieu, and Hansteen, 2012), increased flux emergence rates into the corona, reduced Alfvén wave flux from photospheric foot-point motion, and changes in the structure of MHD shocks, prominences, and quiet-Sun magnetic features (see Anan, Ichimoto, and Hillier, 2017, and the references therein). Observational verification of the reconnection implications of partial ionization is more readily achieved in these solar contexts than in more distant astrophysical settings.

\subsection{Waves in the Solar Atmosphere}

What wave modes are present at what heights in the solar atmosphere? What are their sources? What role do waves play in chromospheric and coronal heating? How do the answers to these questions depend on the local magnetic-field structure?

At least two implications promote the use of DKIST to study magnetohydrodynamic waves in the solar atmosphere (e.g. Kostik and Khomenko, 2013): i) MHD waves carry energy into the solar atmosphere and, if dissipated at the correct heights, may provide at least a partial solution to the long-standing coronal and chromospheric heating problems, and ii) the presence of magnetic fields in the chromosphere and corona modify the waves observed, providing a possible opportunity to use them as diagnostics of the conditions there. The chromosphere is a particularly important region of the solar atmosphere, as it modulates wave transmission into the corona; understanding the solar chromosphere is critical to constraining the mechanisms of wave-energy transfer between the photosphere and the corona (see the review by Jess et al., 2015). Moreover, while it is much cooler than the corona, the chromosphere's relatively high density and the efficiency of the cooling pathways available there mean that high-energy input is required to sustain radiative losses. Typical radiative losses are estimated to be on the order of $10^{6}-10^{7} \mathrm{erg} \mathrm{cm}^{-2} \mathrm{~s}^{-1}$ in the chromosphere compared to $10^{4}-10^{6} \mathrm{erg} \mathrm{cm}^{-2} \mathrm{~s}^{-1}$ in the solar corona (Withbroe and Noyes, 1977; Anderson and Athay, 1989). The solution to the coronal-heating problem may well be coupled to or depend on solution of the chromospheric heating problem (e.g. Carlsson, De Pontieu, and Hansteen, 2019).

The possibility that the Sun's chromosphere and the corona are heated by the dissipation of MHD waves has led to a substantial body of research, starting over 70 years ago with suggestions that acoustic waves generated by convection are responsible for heating the solar atmosphere (Biermann, 1946; Schwarzschild, 1948). With the observational discovery (Leighton, 1960; Leighton, Noyes, and Simon, 1962) and correct theoretical interpretation (Ulrich, 1970) of the resonant solar acoustic oscillations, the diagnostic potential of the solar $p$-modes in the study of the solar interior was realized with helioseismology. This was closely followed by an understanding of the observational and theoretical distinction between those modes and propagating atmospheric waves (see Stein and Leibacher, 1974 , for an early summary). In the intervening years, studies of chromospheric and coronal waves have leveraged increasingly sophisticated space- and ground-based instrumentation, and the consequent ever-increasing spatial, spectral, and temporal resolution observations, to advance our understanding of chromospheric and coronal wave behavior. Yet fundamental 
questions about wave-energy transport and wave heating of the solar atmosphere persist (e.g. Erdélyi and Fedun, 2007). What processes dominate wave generation? What modes are excited? How does the energy generated propagate into the solar corona? What is the role of mode conversion? What are the wave-dissipation mechanisms that allow the solar corona to maintain its multi-million-kelvin temperature?

Answering these questions requires the tracking of waves with height in the solar atmosphere, while simultaneously diagnosing changes in wave energy and the corresponding localized atmospheric heating (Jess et al., 2015). In combination with space-based UV observations, DKIST's unprecedented multi-height spectropolarimetric measurements will be ideally suited to unraveling the nature of the waves, the energy propagation channels accessed, and the atmospheric heating that results.

In a uniform plasma under the continuum approximation, there are three distinct types of MHD wave modes: the slow and fast magneto-acoustic waves and Alfvén waves. Solar observations are often interpreted in terms of these, but this is a dramatic over-simplification, as the manifestation of these waves in the stratified and highly magnetically structured solar atmosphere is much more complex than in a uniform plasma (e.g. Banerjee et al., 2007; Tomczyk et al., 2007; De Pontieu et al., 2007b; Jess et al., 2009; Morton et al., 2012). In simple isolated magnetic geometries such as slabs or flux tubes, modes of the magnetic structures themselves (surface, body, kink, sausage, etc.) can be identified (e.g. Roberts, 1981a,b; Edwin and Roberts, 1982, 1983), but in general, with space-filling magnetic fields, the modes are mixed and coupled, and the waves are subject to resonant absorption, phase mixing, and guided propagation (e.g. Heyvaerts and Priest, 1983; Nakariakov et al., 1999; Bogdan, 2000; Nakariakov and Verwichte, 2005; Aschwanden, 2006; De Moortel, 2009; Jess et al., 2009; Ruderman and Erdélyi, 2009; Goossens, Erdélyi, and Ruderman, 2011; Morton et al., 2011; Wang, 2011; Mathioudakis, Jess, and Erdélyi, 2013; Priest, 2014; Okamoto et al., 2015; Antolin et al., 2015a; Keys et al., 2018). This makes observations difficult to interpret, but also consequently rich in diagnostic potential.

Additional complexities originate with the sate of the solar chromospheric plasma. While the coronal plasma can be treated as a single, low plasma- $\beta$, fully ionized fluid, the chromosphere is a multi-fluid, partially ionized medium, with a finite spatially varying plasma- $\beta$, coupled to a radiation field that is out of thermodynamic equilibrium (e.g. Hansteen, Carlsson, and Gudiksen, 2007). Waves in the solar chromosphere are subject to a highly structured (on sub-arcsecond to global scales) evolving field and flow (e.g. Wedemeyer-Böhm, Lagg, and Nordlund, 2009). Since the manifestations and behaviors of magneto-hydrodynamic waves differ in different magnetic and plasma regimes (high $\beta$ vs. low $\beta$, structured vs. unstructured (on the scale of a wave) field, partial vs. full ionization, as examples), wave signatures change as the waves propagate upward into the solar atmosphere, and tracking the wave energy from its source to the site of energy deposition is challenging.

Despite these difficulties, significant progress has been made and is anticipated with future observations (e.g. Banerjee et al., 2007). Convective motions in the photosphere excite both longitudinal and transverse perturbations. Of the compressive wave field excited in the photosphere, only waves above the temperature-minimum acoustic cut-off frequency are expected to propagate into the atmosphere above, steepening, shocking, and dissipating as they propagate into the chromosphere (Zhugzhda, Bromm, and Ulmschneider, 1995; Carlsson and Stein, 1997). On the other hand, transverse Alfvénic perturbations can propagate through the chromosphere and into the corona with only weak damping. This allows these waves to reach greater heights but also makes their role in plasma heating somewhat problematic. One possibility is that the waves undergo mode conversion from Alfvénic to compressive, with the compressive motions providing an avenue for dissipation and heating (e.g. Hollweg, Jackson, and Galloway, 1982; Ulmschneider, Zähringer, and Musielak, 

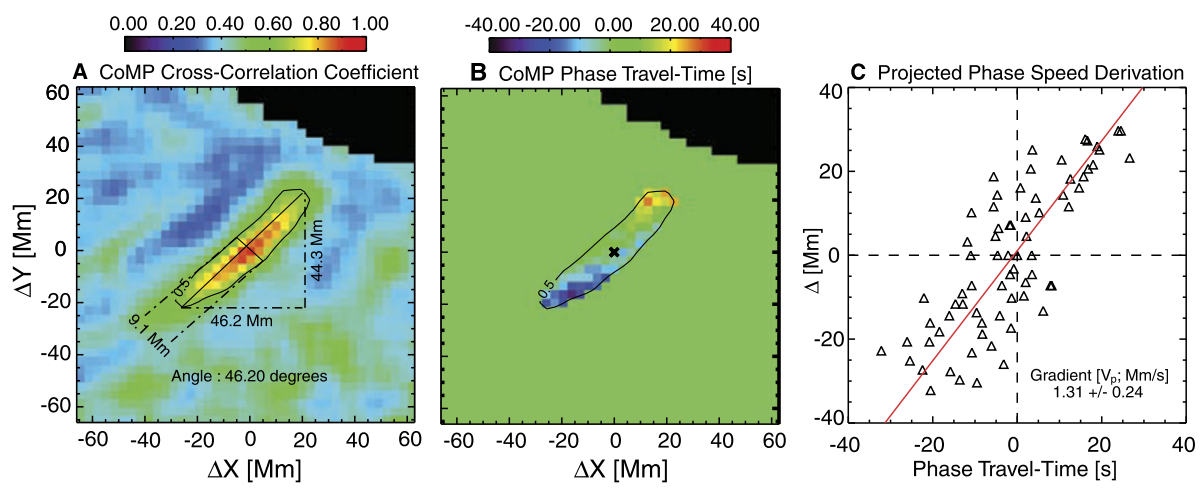

Figure 12 Travel-time analysis of CoMP Doppler velocity measurements. In (A), a map of the cross correlation between the Fourier filtered time series (Gaussian filter centered on $3.5 \mathrm{mHz}$ ) at the reference pixel (marked with an $\times$ in $\mathbf{B}$ ) and the filtered time series of neighboring pixels. The contour of 0.5 defines the region within which the phase speed of the waves is measured to determine the phase travel times shown in $(\mathbf{B})$. In (C), the relationship between the phase travel time and the distance to the reference pixel, yielding the phase speed of the analyzed region. From Tomczyk et al. (2007), copyright by AAAS.

1991; Kalkofen, 1997). The height at which mode conversion occurs is then critical to the height-dependent wave fluxes and consequent heating of the upper atmospheric layers.

Observations of compressive wave motions support this general picture with some modification. High-frequency power (near three minutes in period) dominates the chromospheric wave field as expected, but it does so only in limited inter-network regions devoid of strong photospheric or chromospheric-canopy fields (Vecchio et al., 2007; Vecchio, Cauzzi, and Reardon, 2009). In plage regions, compressive wave power in the chromosphere reaches a maximum well below the acoustic cut-off frequency (e.g. Centeno, Collados, and Trujillo Bueno, 2009), and low-frequency magneto-acoustic waves propagate upward from the photosphere in regions surrounding network elements (Jefferies et al., 2006; Vecchio et al., 2007). The presence of magnetic field thus appears to dramatically influence the frequency of the waves propagating into atmosphere. Several mechanisms have been proposed to facilitate this (Roberts, 2000, 2006): inclined magnetic field can effectively act as a waveguide to reduce the cut-off frequency (Michalitsanos, 1973; De Pontieu, Erdélyi, and James, 2004) and, even for vertically oriented field, nonadiabatic waves can be evanescent outside of magnetic flux tubes yet propagate within them (Roberts, 1983; Centeno, Collados, and Trujillo Bueno, 2006; Khomenko et al., 2008; Centeno, Collados, and Trujillo Bueno, 2009). These mechanisms can be distinguished by careful measurement of the magneticfield structures supporting the waves and wave temperature-velocity phase relations (Kostik and Khomenko, 2013, but cf. Heggland, De Pontieu, and Hansteen, 2009). More generally, MHD wave properties, and the propagation characteristics and amplitudes of waves of different frequencies, depend in detail on the magnetic structure of the region, radiative energy exchange, and the ionization state of the plasma (Khomenko et al., 2018).

Direct detection of Alfvén-wave perturbations is even more challenging than detection of compressive waves, but Alfvén waves have been successfully observed in the chromosphere (De Pontieu et al., 2007b; Jess et al., 2009), transition region (De Pontieu et al., 2014a), and solar corona, both as time-varying non-thermal line widths (see review, Mathioudakis, Jess, and Erdélyi, 2013) and directly as linear-polarization and Doppler velocity fluctuations (Figure 12, Tomczyk et al., 2007, but cf. Van Doorsselaere, Nakariakov, and Verwichte, 2008). The spectrum of the motions observed in the corona has similarities 

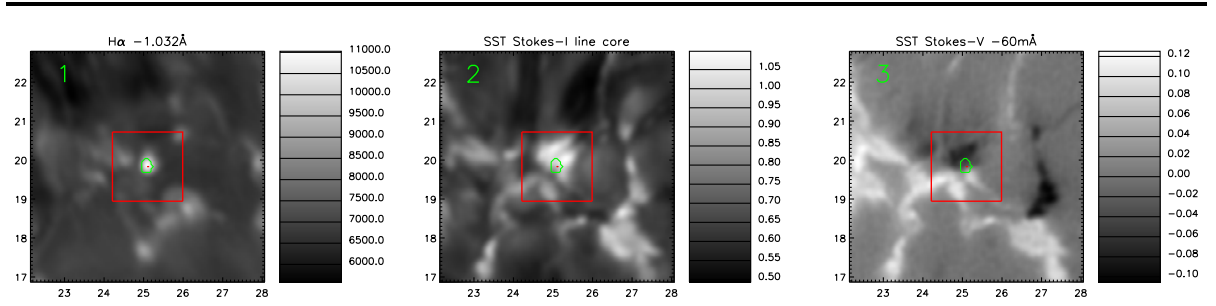

Figure 13 Co-spatial imaging of an Ellerman bomb (CRisp Imaging Spectro-Polarimeter (Scharmer et al., 2008) at the Swedish Solar Telescope (Scharmer et al., 2003)): H $\alpha$ intensity $1.032 \AA$ short ward of line center (left), Fe I $6302 \AA$ line core Stokes-I (middle), and Fe I $6302 \AA-60$ m Stokes- $V$ (right). Ellerman bombs occur near solar active regions or in areas of enhanced photospheric magnetic activity when emerging flux interacts with pre-existing opposite polarity field. Axes units are arcseconds. From Reid et al. (2016), copyright by AAS.

with that of the solar $p$-modes, suggesting the waves have their origin in the solar photosphere (Tomczyk and McIntosh, 2009; Morton, Weberg, and McLaughlin, 2019), although other evidence indicates that they may be more closely related to the ubiquitous Alfvén waves observed in spicules (De Pontieu et al., 2007b; McIntosh et al., 2011a). Observational evidence for mode coupling between fast and slow modes has also been reported. Upward-propagating transverse motions coupled to longitudinal motions subsequently dissipated were identified in quiet-Sun network bright points by careful cross correlation between wavelet-identified wave packets at multiple heights (McAteer et al., 2003; Bloomfield et al., 2004), and evidence for photospherically generated longitudinal magneto-acoustic oscillations propagating upward (Freij et al., 2014) before undergoing mode conversion to predominantly transverse motions has been found in observations of spicules (Jess et al., 2012).

While these interpretations are compelling, to fully disentangle the signal of the different wave modes as they travel upward through the complex solar atmosphere from the photosphere to corona requires simultaneous intensity, polarimetry (magnetic field), and Doppler measurements at many heights in order to deduce the background magnetic field, thermodynamic state of the plasma, and wave perturbations. Such measurements are required for different magnetic-field regimes in order to assess the range of behaviors and quantify the wave fluxes with height. The high-resolution limb spectropolarimetry needed to achieve these measurements is difficult because the low photon counts off the solar limb limit the accuracy of the Stokes profiles deduced at the required cadence and because the low photon counts make the use of adaptive optics, needed to achieve the high resolutions required, challenging. DKIST is poised to meet these challenges. Its large aperture and coronographic capabilities will allow for spectropolarimetric measurements of chromospheric and coronal waves on unprecedentedly small spatial scales and with high cadence.

\subsection{Flux Emergence into the Non-eruptive Solar Atmosphere}

How does magnetic-flux emergence impact energy storage and release in the chromosphere and corona? How are the underlying magnetic-reconnection geometries and heights reflected in observations of small-scale flux emergence/cancelation events?

Magnetic-flux emergence allows for mass, energy, and magnetic field to flow from the solar interior through the photosphere and into the chromosphere and corona above. The impact of flux emergence depends on the amount of field emerging, its spatial distribution, 
and the pre-existing structure of the atmosphere into which it is emerging. A small bipole will have vastly different impact when emerging into a coronal hole, into the quiet Sun, or adjacent to a $\delta$-spot active region. The emergence of magnetic field through the solar photosphere and its reconnection with pre-existing field at different heights thus leads to a variety of dynamic phenomena on different temporal and spatial scales: global magneticfield restructuring (Török et al., 2014), flaring active regions (Toriumi and Wang, 2019), emerging flux regions or arch filament systems (e.g. Centeno et al., 2017; Su et al., 2018), Ellerman bombs (Figure 13), and quiet-Sun Ellerman bomb-like brightenings. Larger-scale field reconfiguration and destabilization was considered in Section 4.2. The discussion in this section is focused on flux emergence on scales below those of active regions and the local response to that emergence. Tracking the consequences of flux emergence on these small scales allows us to understand the fundamental energy storage and release mechanisms that may be responsible for heating the chromosphere and corona.

Ellerman bombs (Ellerman, 1917) are point-like brightenings seen in the wings of chromospheric lines (such as those of $\mathrm{H} \mathrm{I}$ and Ca II). They are often associated with bipolar moving magnetic features around well-developed sunspots or as colliding bipolar structures in emerging flux regions (e.g. Rutten et al., 2013; Reid et al., 2016). The atmospheric heating and associated bi-directional flows observed are thought to be caused by magnetic reconnection near the temperature minimum (e.g. Matsumoto et al., 2008), with rapidly evolving flame-like features in the wings of the Balmer $\alpha$ line suggesting reconnection of small-scale fields when observed at high resolution (Watanabe et al., 2011). While Ellerman bombs primarily occur in active regions, and are sometimes associated with arch-filament systems (e.g. Zachariadis, Alissandrakis, and Banos, 1987; Georgoulis et al., 2002; Ma et al., 2015, but see Rutten et al., 2013, who suggest these are distinct phenomena), suggesting that largerscale flux eruption may underlie their occurrence (Pariat et al., 2004), recent observations indicate that smaller, shorter-lived, and lower $\mathrm{H} \alpha$-wing-intensity-contrast events also occur in the quiet Sun. These quiet-Sun Ellerman-like brightenings (Rouppe van der Voort, Rutten, and Vissers, 2016; Shetye et al., 2018) are similar to Ellerman bombs, but with typical size scales of $\approx 0.25-0.5$ arcseconds and lifetimes of less than a minute compared to arcseconds and minutes for Ellerman bombs proper (e.g. Roy and Leparskas, 1973; Kurokawa et al., 1982; Vissers, Rouppe van der Voort, and Rutten, 2013). Bright flame-like emission in the wings of $\mathrm{H} \alpha$, similar to that observed for Ellerman bombs, suggests a common reconnection origin, but the heating profile and the characteristics of the magnetic-field evolution may imply a somewhat different reconnection scenario (Rouppe van der Voort, Rutten, and Vissers, 2016). Similarly, UV bursts appear to be reconnection events that differ from both Ellerman bombs and quiet-Sun Ellerman-like brightenings in their magnetic topology, atmospheric penetration height, and energy, with the plasma in these events heated to transition-region temperatures (Nelson et al., 2017a; Young et al., 2018; Ortiz et al., 2020).

This full range of small-scale flux-emergence events is well suited for study with the planned DKIST instrument suite. High-resolution, multi-thermal, moderate field-of-view observations can resolve the finely structured thermal properties of the plasma with height, and high-sensitivity spectropolarimetric observations can be used to determine the heightdependent vector magnetic field and Doppler velocities. These quantities in turn will allow estimates of the local electric fields and energy fluxes. Electric-field measurements, derived from time series of vector magnetic-field and Doppler-velocity maps (e.g. Fisher, Welsch, and Abbett, 2012; Kazachenko, Fisher, and Welsch, 2014), are important for determining the rate of electromagnetic-energy transport into the solar atmosphere: the Poynting flux through the photosphere. To date, such measurements have been made only in strong-field 
regions due to the limited reliability of vector magnetic-field deductions in weak-field regions (Kazachenko et al., 2015). Using DKIST's unprecedented vector magnetic-field measurement capabilities, electric-field determination can be dramatically improved, and when combined with transition-region and coronal observations can be used to address a range of open questions about the net transfer of magnetic energy into the solar atmosphere: How much magnetic energy reaches the chromosphere? Why are active region cores the sites of the hottest and most dense coronal loops? Is there a measurable correlation between the input of energy at the photosphere and consequent emission in the chromosphere, transition region, and corona? Is the injected energy dissipated immediately, or stored with some typical latency time? Importantly, with DKIST these questions can be addressed as a function of solar activity to uncover the underlying energetics of the solar cycle.

A recent study of a UV burst with the Sunrise balloon-borne telescope (Smitha et al., 2018) revealed dynamic substructure on scales of $75 \mathrm{~km}$, and likely smaller, within a chromospheric heating site. DKIST will not have UV capabilities, but the He ID3 and $1083.0 \mathrm{~nm}$ lines may serve as useful proxies when studying plasma at transition-region temperatures (Libbrecht et al., 2017). Coordinated observations with space-based UV assets are also anticipated, and numerical models will play a critical role in data interpretation. Radiative magneto-hydrodynamic simulations of magnetic reconnection during flux emergence show reconnection events similar to Ellerman bombs and other burst events (Danilovic, 2017; Hansteen et al., 2017). The occurrence of these in simulations of emerging active regions suggests a possible role for an underlying emerging large-scale twisted loop structure (e.g. Isobe, Tripathi, and Archontis, 2007; Archontis and Hood, 2009). Detailed modeling of expected DKIST spectropolarimetric measurements in the photosphere and chromosphere will enable careful comparisons between the simulated and observed plasma flows and magnetic-field evolution at the Ellerman-bomb sites (Socas-Navarro et al., 2006; Kondrashova, 2016) to determine if the existence of such large-scale structures is implicated by observations of the Sun. Additionally, direct detection of the brightness temperature excess at Ellerman-bomb sites and in the surrounding atmosphere will be possible with coordinated observations using DKIST and radio instruments such as the ALMA. These will allow careful assessment of magnetic-reconnection heating efficiency in the partially ionized chromospheric plasma, and help clarify the overall importance of Ellerman bombs and other burst and localized brightening events to the energy budget.

\subsection{Multilayer Magnetometry and Magnetic-Field Extrapolation}

How does the magnetic-field change with height and evolve in time through different layers of the solar atmosphere? How do we best use multi-layer magnetic-field observations to constrain chromospheric/coronal field extrapolations?

The stratified solar atmosphere is threaded by magnetic field. Most existing solar instruments employ one or a few spectral lines at a time and thus probe the magnetic field over a limited range of heights in the atmosphere. Moreover, the rapid decrease of the magneticfield intensity with height implies very weak chromospheric-polarization signals, making their measurement challenging with existing facilities (Schad, Penn, and Lin, 2013). A common need underlying much of the first critical science proposed for DKIST is multi-line high-sensitivity spectropolarimetry. In meeting this need, DKIST will enable simultaneous multi-height measurements of the solar atmosphere that will revolutionize our understanding of the coupling between atmospheric layers.

The solar chromospheric plasma is highly dynamic, inhomogeneous, and out of local thermodynamic equilibrium. Magnetic fields play a central role in its behavior. Observations 
(a)

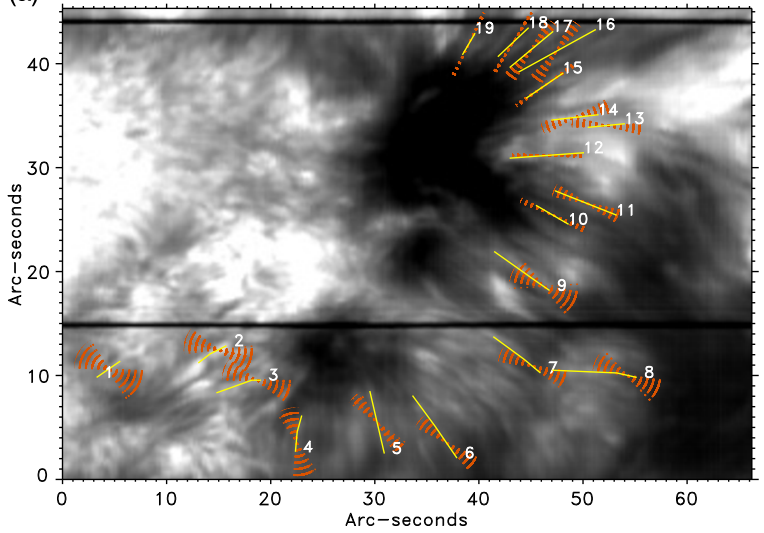

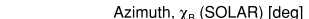

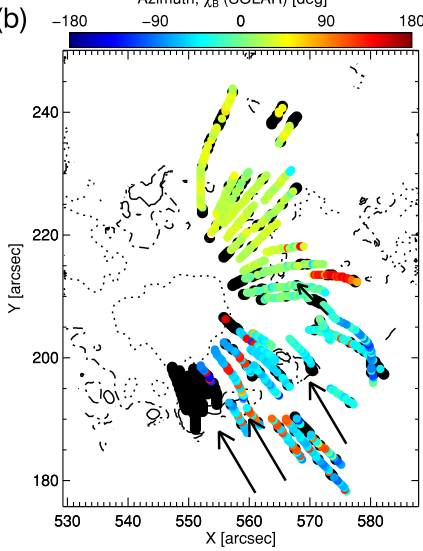

Figure 14 Orientation and azimuthal-field direction of super-penumbral fibrils. In the left panel, yellow lines trace the core Ca II $854.2 \mathrm{~nm}$ intensity and orange cones show the range of transverse magnetic-field azimuth compatible with the linear-polarization measurements. In some regions the fibril and horizontal-field orientations appear aligned (fibrils 9-19), while in others they do not (fibrils 1-5). From de la Cruz Rodríguez and Socas-Navarro (2011), reproduced with permission; copyright 2011 European Southern Observatory. In the right panel, magnetic-field azimuth along selected super-penumbral fibrils (inferred from He I $1083.0 \mathrm{~nm}$ observations). Field azimuth in chromospheric fibrils is generally consistent with those of the penumbral filaments below (not shown). Black dots mark locations of significant deviation. They are largely restricted to sites where fibrils are rooted. From de la Cruz Rodríguez and Socas-Navarro (2011), copyright by ESO. From Schad, Penn, and Lin (2013), copyright by AAS.

of the upper chromosphere, particularly near active regions, are dominated by intricate filamentary structures called fibrils. These fibrils are seen in images taken in the cores of strong chromospheric lines, such as $\mathrm{H} \alpha$, Ca II K, the Ca II IR triplet (e.g. Hansteen et al., 2006; Cauzzi et al., 2008; Pietarila et al., 2009), and the He I 587.6 and $1083 \mathrm{~nm}$ lines (Schad, Penn, and Lin, 2013). They are assumed to be aligned with the magnetic field, but finescale chromospheric filamentary structure is detected even above sunspots, where the photospheric umbral field is strong, thought to be largely vertical, and quite uniform; umbral flashes show filamentary fine structure and apparent associated horizontal magnetic field, at or below the scale of current spatial-resolution limits (e.g. Socas-Navarro et al., 2009). Is this an indication of complex field structure or an indication that using fibrils as an indicator of field direction is problematic?

The assumed alignment of chromospheric fibrils with the magnetic field is an important tool in active-region field extrapolation (Wiegelmann et al., 2008; Jing et al., 2011; Yamamoto and Kusano, 2012), and accurate field extrapolation is critical to assessments of the free energy available for solar flares and eruptions, active-region flaring potential, and stability, and models of chromospheric heating. However, ever since the early conjecture by George Ellery Hale that fibrils around sunspots reflect lines of magnetic force (Hale, 1908b), a conjecture made even before his momentous measurement of the field using the then recently described Zeeman effect (Hale, 1908a, see Harvey, 1999, for a brief history), the observational evidence for a direct association between fibrils and the local magneticfield direction has remained sparse. Because of the small amplitude of the polarization signals within the primarily horizontally oriented (relative to the solar surface) chromospheric fibrils $(<0.1 \%$ in linear polarization), conclusions are wide ranging. For example, attempts to directly measure the alignment between the thermal and magnetic structure of super-penumbral fibrils (Figure 14) have yielded results ranging from often, but not always, 
aligned (de la Cruz Rodríguez and Socas-Navarro, 2011) to aligned within \pm 10 degrees with no evidence for misalignment (Schad, Penn, and Lin, 2013). A recent Bayesian statistical analysis (Asensio Ramos et al., 2017) finds penumbral and plage fibrils to be well aligned but with non-negligible dispersion. That study concludes that higher signal-to-noise observations are needed to discern whether the misalignment seen in some simulations, particularly those that include ion-neutral coupling (Martínez-Sykora et al., 2016), is compatible with that seen on the Sun. Understanding the three-dimensional connectivity of active-region chromospheric field to the photosphere (at sites such as the outer foot points of superpenumbral fibrils; Schad, Penn, and Lin, 2013) requires high-resolution, high-sensitivity spectropolarimetric measurements. DKIST will enable these.

The connectivity of the magnetic field through the atmosphere is an important issue outside of active regions as well. In the quiet Sun, the photospheric magnetic field is organized by supergranular motions into strong flux concentrations on the network scales and mixedpolarity inter-network magnetic field on the scale of granulation. The field expands above the photosphere into the chromosphere and corona, and the presence of the weak small-scale inter-network magnetic field has a considerable effect on the overall field geometry with height, which deviates significantly from a simple funnel-expansion model (Schrijver and Title, 2003; Aiouaz and Rast, 2006; Martínez-Sykora et al., 2019). This is critical because the magnetic field forms the underlying channel for energy transport into the solar chromosphere and corona, playing an important role in the acceleration of the solar wind (e.g. Gabriel, 1976; Aiouaz, Peter, and Lemaire, 2005; McIntosh et al., 2007; Tian et al., 2008, see also previous sections in this Research Area). Beyond idealized potential or force-free field extrapolations, the variation in field strength and topology with height is typically poorly known.

Force-free extrapolations can be improved. Because of the availability of photospheric magnetograms, field extrapolations generally depend on photospheric boundary conditions, but these boundary conditions are inconsistent with the force-free assumption because both gas pressure and gravity play important roles at photospheric heights. Employing chromospheric-field measurements instead, measurements made at sufficiently great heights, where the magnetic field is much more dominant and is consequently configured much closer to a force-free state (Zhu et al., 2016), can significantly improve the reliability of field extrapolations (Fleishman et al., 2019); when combined with photospheric measurements, even very limited chromospheric-field measurements allow significant improvement (Fleishman et al., 2019). Additionally, careful comparison between independent extrapolations using photospheric- and chromospheric-field measurements can aid in determining relative line-formation heights and in resolving the 180-degree field ambiguity (Yelles Chaouche et al., 2012), and reliable multi-height magnetic-field measurements with DKIST will not only contribute to more reliable extrapolation of that field but will strengthen deductions of the local field at the measurement site.

\subsection{Magnetic Topology, Helicity, and Structures}

What role does the near conservation of helicity play in the structuring of the solar corona and coronal mass ejections? Are measurements of helicity useful indicators of imminent eruption? Do vortex tubes exist and do they act as portals for MHD wave propagation and energy transfer in the quiet solar atmosphere? What are the magnetic-field properties of vortex structures in the lower solar atmosphere?

Magnetic helicity is a property of the field that helps describe its topology, whether it is twisted or linked, writhes or is sheared (e.g. Moffatt, 1969; Berger, 1999; Moffatt, 2014; 
Blackman, 2015; Moffatt, 2016). It is strictly conserved in ideal MHD (Woltjer, 1958) and during two-dimensional reconnection, and approximately conserved after three-dimensional reconnection (Taylor, 1974; Berger, 1984; Hornig and Rastätter, 1997). Magnetic helicity cascades to larger scales (e.g. Frisch et al., 1975; Pouquet, Frisch, and Leorat, 1976; Pouquet and Patterson, 1978; Alexakis, Mininni, and Pouquet, 2006) and is converted from one form to another (between twist and writhe for example) as it moves to larger scales (e.g. Pevtsov et al., 2014; Knizhnik, Antiochos, and DeVore, 2017; Scheeler et al., 2017; Zuccher and Ricca, 2017). This means that as magnetic fields reconfigure in the solar atmosphere, magnetic helicity is lost only slowly.

Many solar magnetic structures contain self-helicity (internal twisting) and/or mutual helicity (tangling about each other), with helicity observed on the Sun on scales ranging from the largest global to the smallest quiet-Sun magnetic fields (e.g. Pevtsov and Balasubramaniam, 2003; Welsch and Longcope, 2003, and the references therein). The intense magnetic-field structures that form within and rise through the Sun's convection zone, and are thought to be responsible for sunspots and most solar activity, are likely highly twisted. Untwisted, such tubes would lose their integrity as they ascend. Observations of sunspots show that they rotate as they emerge (e.g. Evershed, 1909; Brown et al., 2003). That rotation is likely associated with an underlying large-scale twisted flux tube rising through the photosphere (e.g. Sturrock et al., 2015), and the helicity that enters the solar atmosphere on all scales from below has important implications for the structure and behavior of the field there.

Some coronal loops appear to be tangled about each other forming a braided pattern (Parker, 1983; Cirtain et al., 2013; Pontin et al., 2017), and the degree of coronalloop braiding overall has been used to estimate the role of small-scale reconnection in coronal heating (Schrijver, 2007; Knizhnik, Antiochos, and DeVore, 2017). The appearance of braided structures, however, depends critically on the details of the field-line windings within them, with loop substructure difficult to distinguish in observations (Berger and Asgari-Targhi, 2009; Pontin et al., 2017; Li and Peter, 2019), so careful high-resolution spectropolarimetric observations are vital. Further, contrary to expectation, coronal loops have quite uniform width along their length (Klimchuk, 2000; Watko and Klimchuk, 2000). Explanations for the observed lack of expected field expansion rely on loop substructure, either to provide magnetic tension (e.g. López Fuentes, Klimchuk, and Démoulin, 2006) or to allow fine-scale interchange reconnection that enables cross-field loss of the hot loop plasma (Schrijver, 2007; Plowman, Kankelborg, and Longcope, 2009). Untangling these observationally, by leveraging DKIST's coronal magnetic-field measurement capabilities, is important in understanding these thermodynamic structure of the corona and its maintenance.

Beyond loop substructure and heating, the accumulation of magnetic helicity in the corona appears to be of key importance to coronal mass ejections. Magnetic-helicity accumulation accompanies the magnetic-energy build-up that precedes the loss of stability when a coronal mass ejection is initiated (Zhang and Low, 2005; Zhang, Flyer, and Low, 2006; Yeates and Hornig, 2016). The precise stability implications of the helicity accumulation are still somewhat uncertain (Amari et al., 2003; Phillips, MacNeice, and Antiochos, 2005), and some measures of helicity may be more reliable instability indicators than others (Pariat et al., 2017), but independent of the exact triggering mechanisms, coronal mass ejections associated with filament eruptions often reveal large-scale helical magnetic structures that partially unwind during the eruption (e.g. Kurokawa et al., 1987; Xue et al., 2016). Coronal mass ejections may thus play an essential role in relieving the solar atmosphere of accumulated helicity (Zhang, Flyer, and Low, 2006), and detailed observational assessment of the 
Figure 15 The "Grand Daddy Prominence." Photographed by W.O. Roberts at the Harvard College Observatory, Climax, Colorado on 4 June 1946 through a filter centered on $\mathrm{H} \alpha$. This prominence extends about $200,000 \mathrm{~km}$ above the solar surface. Courtesy of the High Altitude Observatory/NCAR (www2.hao.ucar.edu/Education/ Sun/grand-daddy-prominence).

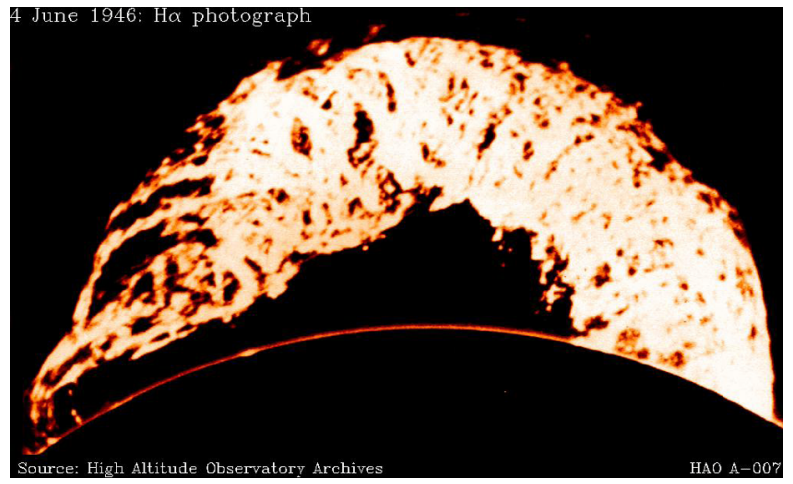

coronal helicity budget and its role in coronal mass ejection initiation are crucial. Moreover, highly twisted structures typically have a high magnetic energy, and that twist can lead to local instability (e.g. the kink instability), reconnection, flaring, and small-scale eruptions in addition to coronal mass ejections proper. DKIST will significantly enhance our ability to deduce the magnetic helicity in pre-and post-eruptive magnetic fields.

Prominences (or filaments on the disk) are cool plasma structures (at chromospheric temperatures) embedded into the hot corona (e.g. Parenti, 2014; Gibson, 2018). They are observed in emission off-disk (Figure 15) and as filaments in absorption when observed on-disk. These helical structures are central to those coronal mass ejections associated with filament eruptions, but direct measurements of their magnetic-field topology are limited (e.g. Casini et al., 2003; Xu et al., 2012; Kuckein, Martínez Pillet, and Centeno, 2012; Sasso, Lagg, and Solanki, 2014). Based on magnetostatic models and plasma support and stability considerations, the magnetic field in prominences is thought to be fundamentally tangential to the solar surface. This may be true even in the feet (barbs) of the prominence, which can be the sites of swirling motions sometimes called solar tornadoes (e.g. Panesar et al., 2013; Levens et al., 2016a,b; Mghebrishvili et al., 2015, 2018). Sometimes the plasma flows in opposite directions (known as counter-streaming/bi-directional flows) along the spine of filaments, as well as in barbs. The origin of these bi-directional flows is still not well understood (Zirker, Engvold, and Martin, 1998; Panesar et al., 2020). They may reflect the rise and expansion of a twisted flux rope into the corona or the presence of a large vortex flow in the photosphere (see Mghebrishvili et al., 2015, and the references therein), but while it is unclear which interpretation is correct, the motions are implicated in prominence stability (Mghebrishvili et al., 2018). Understanding these complex evolving dynamics is critical to assessing the role of prominences in the solar mass cycle (Section 5.1) and coronal mass ejection initiation (Section 4.1).

Magnetic helicity in the solar atmosphere has two sources, the emergence of helical field through the photosphere from below (e.g. Leka et al., 1996; Tian and Alexander, 2008) and field foot-point motions due to photospheric flows (e.g. van Ballegooijen, 1999; Chae, 2001) including differential rotation (e.g. van Ballegooijen, 1999; DeVore, 2000). Observations aimed at understanding the atmospheric helicity budget can either focus on these sources of helicity or attempt a direct measurement of the helicity in the solar atmosphere itself (see reviews by van Driel-Gesztelyi, Démoulin, and Mandrini, 2003; Démoulin, 2007; Démoulin and Pariat, 2009, or more recent references in Linan et al., 2018). To date, determination of the magnetic-field helicity in the solar atmosphere has relied on field extrapolation while estimates of helicity supply have used measurements of the photospheric field and flows and models of their implication for helicity injection into the atmosphere. DKIST observations 
will contribute to the improvement of both of these techniques. DKIST's on-disk, multilayer magnetometry will allow for more direct inference of the sheared and twisted field emerging through the photosphere and present in the chromosphere (e.g. Kuckein, Martínez Pillet, and Centeno, 2012), while DKIST coronal-field measurements will help constrain field-extrapolation methods (Section 5.6) and allow verification of the magnetic-field configurations that result (e.g. Dove et al., 2011; Ba̧k-Stȩślicka et al., 2013). DKIST observations will be regular and sustained, allowing such studies to address active-region evolution and filament formation (Sun et al., 2012).

For decades, braiding and twisting of magnetic field rooted within solar surface convection has been thought to be an efficient mechanism for inducing solar atmospheric heating by reconnection (e.g. Parker, 1972; Klimchuk, 2006; Amari, Luciani, and Aly, 2015). Vortical flows in the photosphere can produce coherent magnetic-field structures, twisted magneticflux tubes, and these can be subsequently braided by random walk of footpoint sites as the flow evolves. Granular downflows in particular can be highly vortical and may consequently play an important role in magnetic-helicity production, reconnection, and MHD wave generation (Stenflo, 1975; Nordlund, 1985; Stein and Nordlund, 2000; Shelyag et al., 2011; Wedemeyer-Böhm et al., 2012; Kitiashvili et al., 2012). In observations of the solar photosphere, vortical motions can be inferred by tracking magnetic bright-points (e.g. Bonet et al., 2008). In the chromosphere, the magnetic field forms swirls, 0.4-2 Mm in diameter, that last for five to ten minutes (e.g. Wedemeyer-Böhm et al., 2012). These appear as rotating spirals in $\mathrm{H} \alpha$ and in the near-IR Ca II $854.2 \mathrm{~nm}$ line, with the near IR signal prominent for small-scale swirls near the solar disk center (Wedemeyer-Böhm and Rouppe van der Voort, 2009) in high-resolution ground-based observations (40 km per pixel at $630 \mathrm{~nm}$ ) using CRisp Imaging Spectro-Polarimeter (Scharmer et al., 2008) at the Swedish Solar Telescope (Scharmer et al., 2003). Observations of heating (Park et al., 2016), swaying (Tziotziou et al., 2018), and wave-like motions (Liu et al., 2019a,b; Shetye et al., 2019) within the spiraling chromospheric swirl structures suggests a rich dynamics that awaits further exploration.

In addition, photospheric vortices have been recently detected in large numbers using local-correlation-tracking techniques (Fisher and Welsch, 2008, and the references therein) on photospheric continuum-intensity images (Giagkiozis et al., 2018; Liu, Nelson, and Erdélyi, 2019). At any one time about $10^{6}$ such photospheric vortices may cover about $2.8 \%$ of the solar surface, although that may be an overestimate (Liu, Nelson, and Erdélyi, 2019), a dispute ripe for resolution using DKIST. Extension of vortex detection to chromospheric heights, and measurement of the correlation between swirls at different heights (Liu et al., 2019b), suggests that they are associated with upward-propagating Alfvén waves. Energy fluxes at the swirl sites may be sufficient to support local chromospheric energy losses if the waves are efficiently dissipated (their global energy contribution depends on better determination of the swirl-site number density). This possiblilty depends on the correlation between the velocity and magnetic field within the swirls. It has been investigated using numerical simulations (Liu et al., 2019a), and is a compelling target for DKIST observations. While most of the observed photospheric vortices have quite short lifetimes (Liu et al., 2019b), the more persistent vortices may more generally serve as important energy portals because they support a wide range of MHD waves. They may also be precursors of the so-called magnetic tornadoes (Wedemeyer et al., 2013) observed in the chromosphere, which in turn may be the source of twist at the foot points of coronal loops that power the continuous basal coronal heating (Wedemeyer-Böhm et al., 2012). Detecting small-scale vortices in the chromosphere is challenging, and the substructure within them has not yet been resolved even in the photosphere. With DKIST, we have the opportunity to determine the number 
and amplitude of compact vortex structures in the photosphere and chromosphere down to very small scales, clarify the dynamical and magnetic connectivity of such structures across atmospheric layers, and assess their collective contribution to MHD wave generation and solar atmospheric heating.

\section{Long-Term Studies of the Sun, Special Topics, and Broader Implications}

The Sun exhibits remarkable changes over decadal time scales, with the spatial distribution of active regions, sunspots, coronal holes, and prominences continuously changing along with the roughly 11-year polarity reversal. The frequency and severity of solar events, such as flares and CMEs, are strongly dependent on the phase of the solar cycle, and the amplitude of the basal high-energy radiative output of the Sun (in the X-ray and EUV) is modulated by orders of magnitude over the course of a solar cycle. These cycle-dependent changes and others hold important clues about the underlying operation of the global solar dynamo.

Cycle-related changes have been observed on the Sun at scales as small as supergranulation; supergranules get larger when the Sun is more active (McIntosh et al., 2011b). It is possible that careful synoptic observations at higher resolution will reveal cycle-dependent dynamics at even smaller scales. Assessing these variations may provide fundamental insights into the multi-scale turbulent dynamics of highly stratified convection. Additionally, global modulation of solar activity occurs on time scales longer than the solar cycle as well. Variations in the strength of solar maxima and the duration and depth of solar minima lead to a cascade of consequences from total- and spectral-irradiance variations to changes in the solar-wind ionization state and mass flux and variations in the complexity of the interplanetary magnetic field and consequent modulation of the cosmic-ray flux into the inner heliosphere. These, in turn, impact the near-Earth and interplanetary-space environments, the Earth's upper atmosphere and to some degree the Earth's climate. Understanding, modeling, and potentially forecasting these impacts requires long-term consistent monitoring of the Sun's magnetic and plasma properties. This is most readily achieved by a ground-based facility, which can maintain the required spatial and temporal resolution and spectropolarimetric sensitivity over decadal time scales because it can be fine-tuned, maintained, and upgraded as needed.

DKIST's unique capabilities will allow for a broad range of investigations beyond the core solar-physics areas that form the bulk of this article. For example, as with previous space-based solar observatories (such as the Solar and Heliospheric Observatory (SOHO), SDO, and the Solar TErrestrial RElations Observatory (STEREO)), DKIST will make contributions to cometary science and the use of comets as probes of otherwise inaccessible regions of the solar corona, regions inaccessible to both remote-sensing observations (too faint to be detected) and direct in-situ measurements (closer than Parker Solar Probe's closest approach to the Sun). As another example, just as atomic physics has benefited from the highresolution spectrometers onboard SOHO (Coronal Diagnostic Spectrometer (CDS) and Solar Ultraviolet Measurements of Emitted Radiation (SUMER)) and Hinode (EUV imaging spectrometer (EIS)), so too will it benefit from DKIST's advanced spectropolarimetric instrumentation and measurements. Previous missions have provided the critical line-intensity measurements necessary to benchmark theoretical predictions of atomic spectra. These have led to major advances in atomic-transition calculations and spectral-synthesis codes, such as CHIANTI, and in turn have allowed for far more accurate predictions of solar X-ray, EUV, and UV spectral irradiance and improved spectral plasma diagnostics. DKIST will similarly 
Figure 16 Polar field strengths from NSO/KP averaged over the latitudes 55-90 degrees (red dots with $1 \sigma$ variance in gray) in the solar northern (top) and southern (bottom) hemispheres.

Dashed-blue curves overplot polar-field measurements from the Wilcox Solar Observatory. Field reversals, determined from $\mathrm{NSO} / \mathrm{KP}$ smoothed data (purple curve), are indicated with blue vertical fiducial lines. The red vertical fiducial line marks the completion of Cycle 24 northern polar reversal. Cycle 24 field reversal was unambiguous in the southern hemisphere but extended over some time in the northern hemisphere. From Janardhan et al. (2018).

Reproduced with permission; copyright 2018 European Southern Observatory.

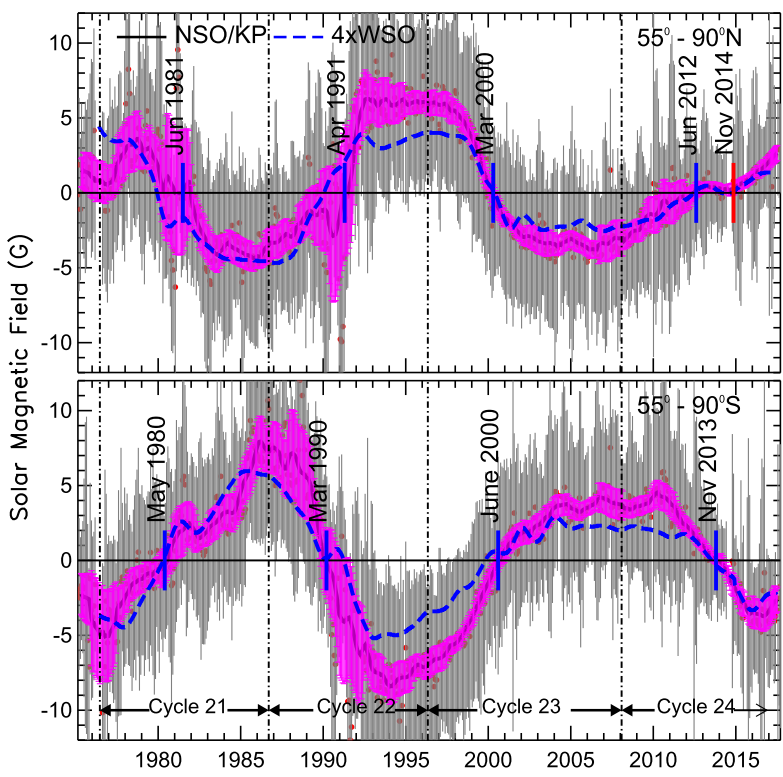

make fundamental contributions to the quantum-mechanical underpinnings of polarization spectroscopy over a broad range of wavelengths extending into the near and mid IR.

Several critical science topics in this research area are discussed in detail below, including i) long-term studies of the Sun; ii) Sun-grazing comets; iii) Mercury-transit science; iv) turbulence and reconnection processes; and v) synergies with in-situ measurements.

\subsection{Long-Term Studies of the Sun}

How do the properties of the Sun's small-scale magnetic field depend on the phase of the solar cycle? Does inter-network magnetism show cycle variations? How do the polar magnetic fields and flows evolve with the solar cycle? Are there systematic changes in prominence/filament fields that reflect a helicity cycle?

DKIST will provide regular, sustained and repeated photospheric, chromospheric and coronal measurements of specific targets using very similar instrumental configurations over many years. The advantages of such repeated long-term observations of the Sun are most evident in the context of the large-scale solar dynamo. Although the global magnetic field of the Sun evolves over the 11-year activity cycle, its detailed behavior likely depends on physical processes that occur on smaller spatial and shorter temporal scales. Many questions regarding the connections between small-scale processes and long-term behavior remain unaddressed due to a lack of high-resolution, high-sensitivity spectropolarimetric observations of the kind that DKIST will make over an extended period of time. DKIST will additionally make regular detailed maps of the coronal magnetic field, a unique observational capability currently missing, and together with coordinated in-situ observations by space missions such as the Parker Solar Probe and Solar Orbiter these will be used to understand the Sun's cycle-dependent influence on the heliosphere. Both types of synoptic observations can be used as benchmarks in the study of activity cycles of other stars (e.g. Brun and Browning, 2017) allowing further contextual understanding of the Sun's behavior. 
Facular-scale magnetic elements are the building blocks of the magnetic field at the solar surface. In some dynamo models they play an essential role in transport, flux cancelation, and field reversal (e.g. Charbonneau, 2010, and the references therein), but systematic study of their motions and mutual interactions over timescales long enough that they are subject to differential rotation and meridional flow (such as those of Lamb, 2017) has not been undertaken in a latitude- and cycle-dependent manner. In particular, facular fields that survive cancelation converge by meridional circulation at polar latitudes (e.g. Tsuneta et al., 2008). There they form large-scale unipolar polar caps, with dynamo implications (Figure 16) and global heliospheric influence (Petrie, 2015), but our knowledge of the details of their distribution, dynamics, and behavior in the polar regions is limited by spatial-resolution constraints associated with foreshortening (e.g. Petrie, 2017).

The spatial-resolution capabilities and polarimetric sensitivity of DKIST are essential in addressing this problem. The multi-instrument capabilities of DKIST will enable polarfield maps at complementary wavelengths (different heights in the atmosphere) and over fields of view and at cadences that maximize the coverage and resolution of long- and shortterm polar-field evolution. These will enable the application of local helioseismic and localcorrelation and structure tracking techniques at high latitudes. With SDO/HMI (resolution 0.5 arcseconds per pixel), solar meridional and zonal flows can be recovered up to latitudes of about 75 degrees. DKIST's better than 0.1 arcsecond resolution and superior sensitivity will allow application of these techniques at latitudes reaching 90 degrees during March and September when the solar poles are most visible. Making such high-resolution nearlimb observations over a sufficiently large field of view will be challenging, but achieving them repeatedly over the course of a solar cycle will yield crucial insights into the timedependent nature of high-latitude meridional and zonal flows, a critical missing piece in our understanding of global flux transport that is highly relevant to its role in the global dynamo process.

Separately, the contribution of small-scale magnetic structures (below the resolution element of current observations) to the total solar irradiance is still not fully understood (discussion in Section 3.3). In particular, the radiative output of magnetic elements, and the spectral distribution of that output, is strongly dependent on the spatial substructure of the elements (Okunev and Kneer, 2005; Criscuoli and Rast, 2009; Uitenbroek and Criscuoli, 2011). Not knowing that substructure introduces significant uncertainty into present-day irradiance models (Peck et al., 2019). Given that quiet Sun covers approximately $90 \%$ of the solar surface and contributes substantially to the disk-integrated magnetic surface flux, changes over the solar cycle in the size distribution or structure of small-scale network and inter-network magnetic elements likely play a significant role in inferred irradiance trends (Harder et al., 2009; Ermolli et al., 2013). Systematic, long-term observations with DKIST, combining the highest spatial-resolution imagery with the highest concomitant polarimetric sensitivity, will be essential in addressing this problem.

Beyond individual magnetic-element contributions, field-induced variations of the temperature gradient in the deep solar photosphere (below $60 \mathrm{~km}$ above the $500 \mathrm{~nm}$ continuum) may contribute to cycle-dependent irradiance variability (e.g. Faurobert, Balasubramanian, and Ricort, 2016, and the references therein). Structural changes are suggested by observations of the acoustic $p$-modes frequencies, which show solar-cycle variation (e.g. Fossat et al., 1987; Libbrecht and Woodard, 1990; Salabert, García, and Turck-Chièze, 2015), and are evident in MHD simulations, which display decreasing temperature in the low photosphere with increasing inter-network magnetic-field strength (Criscuoli, 2013). Observational confirmation by direct measurement of the temperature gradient over the solar cycle is needed, particularly at high resolution so that the role of currently unresolved magnetic 
elements can be elucidated. The method introduced by Faurobert, Balasubramanian, and Ricort (2016) derives the temperature gradient on an absolute geometrical scale based on spectroscopic observations at different heliocentric angles. It is well tailored to exploit highresolution observations, suited to ground-based observations (Faurobert et al., 2018), and it can be readily extended to synoptic DKIST observations in multiple spectral lines.

Synoptic studies of prominences/filaments are also important. Active-region and quietSun prominences/filaments participate in and change with the solar cycle (e.g. Zhang and Low, 2001; Pevtsov et al., 2014; Mackay et al., 2018), and make a critical contribution to space weather via coronal mass ejections (e.g. Kilpua et al., 2019). Filament models usually rely on measurements of the underlying photospheric field and/or emission patterns in the chromosphere, because, although filament magnetic fields have been intermittently measured in the past (e.g. Leroy, Bommier, and Sahal-Brechot, 1983; Xu et al., 2012; Kuckein, Martínez Pillet, and Centeno, 2012; Sasso, Lagg, and Solanki, 2014; Díaz Baso, Martínez González, and Asensio Ramos, 2019a,b), they have not been regularly measured. We thus have only limited understanding of the range of field properties displayed or the variation of those with the solar cycle; no cycle-length synoptic program to directly measure chromospheric filament magnetic fields has yet been undertaken. Such a program will be possible with DKIST. DKIST's multi-height capabilities can be used to clarify the three-dimensional helical magnetic structure of prominences/filaments (Section 5.7) and its evolution, and it will be able to supply space-weather models with direct field measurements. Additionally, it may be possible to extend and enhance space-weather models and predictions by providing measurements of the near-surface flows underlying filaments (Hindman, Haber, and Toomre, 2006). Synoptic, high-cadence and high-resolution observations that are continuous over several hours, in conjunction with high-resolution local-helioseismological analysis, will allow improved measurements of the local subsurface shear layer that is likely central to filament formation, dynamics, and evolution.

More broadly, synoptic DKIST observations of the low solar corona will benefit collaborative science with the Parker Solar Probe and Solar Orbiter missions (Section 6.5 below). The in-situ measurements that these missions provide often require heliospheric-field models for context and interpretation. DKIST's Cryo NIRSP instrument will be able to make direct coronal spectropolarimetric measurements spanning the spacecraft-encounter windows. These measurements can help provide constraints on the heliospheric-field models. While the best input for those models is still uncertain, and line-of-sight integration through the optically thin corona will likely pose some difficulties, measurements of the forbidden Fe XIII line at $1075 \mathrm{~nm}$ will initially be used to diagnose the magnetic field. Measurements will include the Stokes $-V$ component, which is accessible to DKIST because of its anticipated exquisite polarization sensitivity and calibration accuracy.

\subsection{Turbulence and Reconnection Processes}

Where is the turbulence and what causes it? Is there evidence for magnetic-island/plasmoid formation during reconnection in the solar atmosphere? What role do ion-neutral collisions play in these processes?

Complex highly-nonlinear dynamics characterizes the Sun at all scales (Figure 17), and processes at the smallest scales profoundly influence its global behavior. The observational capabilities of DKIST offer the opportunity to study the Sun as a plasma laboratory to investigate the processes of reconnection, turbulence, and dynamo action in the regime of strong nonlinearity, low molecular diffusivity, and partial ionization. 
Figure 17 Prominence eruption/coronal mass ejection observed on 16 April 2012 with SDO/AIA at $30.4 \mathrm{~nm}$. It was accompanied by an M1.7 flare. Courtesy NASA/SDO/AIA. www.nasa.gov/mission_pages/ sunearth/news/News041612M1.7flare.html.

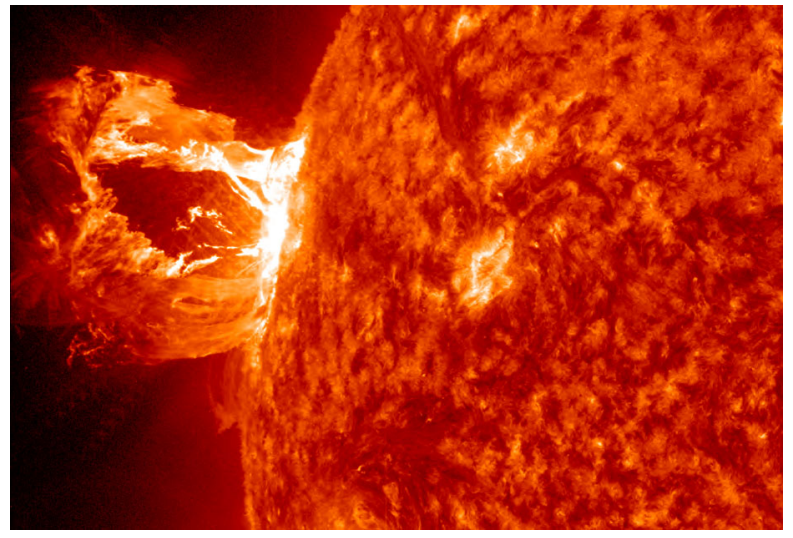

Turbulence is a state of fluid motion that is characterized by unpredictable flow trajectories, a wide range of spatial and temporal scales, and a high degree of vorticity. The fundamental aim of turbulence research is to understand its statistical properties well enough to be able to predict the transport of scalar and vector quantities. While, based on an assessment of molecular transport coefficients (Parker, 1979; Miesch, 2005; Lingam et al., 2017, and references within these), the solar convection zone is very likely highly turbulent below the photosphere, granulation in the photosphere may not be (Loughhead and Bray, 1959; Nordlund et al., 1997). Solar granulation is dominated by the local dynamics of a strongly radiatively cooled, highly stratified boundary layer (e.g. Nordlund, 1985; Rast, 1995; Stein and Nordlund, 1998; Nordlund, Stein, and Asplund, 2009). Upwelling fluid entering the photosphere from below is laminarized by rapid expansion due to the steep mean stratification, and downflowing plumes, initiated in the photosphere, advect flow instabilities out of the readily observable region (Rast, 1998). While the two-dimensional horizontal-transport properties of the observed photospheric flows (e.g. Abramenko et al., 2011; Agrawal et al., 2018) are interesting, critical to some dynamo models (e.g. Charbonneau, 2010, and the references therein), and may contain clues about the convective driving scales at depth (Lord et al., 2014; Cossette and Rast, 2016), at the resolution of current observations these flows show little direct evidence of turbulence.

It is very possible that at DKIST resolution the granular flows will appear significantly more structured than they do at lower resolution. Very high-resolution Doppler imaging with the IMaX instrument (Martínez Pillet et al., 2011) on the first flight of the Sunrise stratospheric balloon (Solanki et al., 2010) revealed that, in small compact regions, granular upflows reach peak speeds approaching those found in downflows (McClure, Rast, and Martínez Pillet, 2019). This substructuring may continue to even smaller scales. Moreover, with DKIST spatial resolution and sensitivity it may be possible to resolve the horizontal gradient of the vertical velocity in the deep photospheric (Khomenko et al., 2010) and/or the onset of turbulent instabilities at the shear interface between the granular upflows and the intergranular downflow lanes. In numerical simulations, these instabilities lead to recirculation of small-scale, mixed-polarity magnetic field several minutes after new downflow plume formation (Rempel, 2018). Observations of the properties of such recirculating flows and fields may provide key constraints on the relative importance of deep and shallow recirculation to the operation of the Sun's small-scale dynamo (Section 3.1), and perhaps more broadly address the detailed structure of the photospheric boundary layer with implications for the amplitude of deep convection below (Rast and Trampedach, 2021). Such work may 
be able to both leverage and augment the rich history of research focused on the Earth's convective planetary boundary layer (e.g. Willis and Deardorff, 1976; Kaimal et al., 1976; Lenschow and Boba Stankov, 1986; Moeng et al., 2009; Sullivan and Patton, 2011; van Heerwaarden, Mellado, and De Lozar, 2014), to better understand how the mean boundarylayer structure is established at the interface between deep convection and discrete downflow (on Earth, upflow) plume initiation. Additionally, although the concept of turbulent spectralline broadening (e.g. Gray, 2005) may not be useful in interpreting high-resolution photospheric observations (Asplund et al., 2000; Khomenko et al., 2010), it is used extensively in the interpretation of stellar photospheric lines (e.g. Sheminova, 2019) to assess unresolved motions. There is some evidence that a more careful assessment of the solar photospheric velocity field could improve stellar photospheric spectral-line modeling (Takeda and UeNo, 2017). Moreover, the concept may still be useful in the case of less than "perfectly" resolved solar observations (Ishikawa et al., 2020), and confident progress toward the elimination of the use of, or the development of more accurate representations of, non-thermal broadening in models of solar chromospheric, transition region, and coronal spectral lines awaits DKIST resolution of small-scale flows in those regions (e.g. Cauzzi et al., 2009; De Pontieu et al., 2015; Leenaarts et al., 2018).

In the so-called "local dynamo" scenario, convective turbulence in the quiet Sun is responsible for the creation and structuring of weak small-scale magnetic field (Section 3.1), with convective motions at the granular and subgranular scales determining the topology of the field and its degree of "entanglement." The Hanle effect is a key tool for investigating this aspect of solar magnetism because the depolarization of scattered photons is sensitive to the presence of small-scale tangled magnetic fields (e.g. Trujillo Bueno, Shchukina, and Asensio Ramos, 2004). The Zeeman effect, by contrast, is blind to mixed-polarity field on scales much smaller than those that can be resolved. Since the amplitude of the Hanle scatteringpolarization signal is very low, the limited resolution and polarimetric sensitivity of current observations only allow determination of an upper limit to its value and spatial variation (e.g. Zeuner et al., 2018). With the increased resolution and polarimetric sensitivity of DKIST, the degree to which the Zeeman and Hanle measurements differ will become a critical measure of the scale at which the field is generated. However, fundamental advances in our understanding of the Hanle signal are required. For example, interpretation of the scatteringpolarization signal at each wavelength depends on knowledge of the anisotropy of the illuminating radiation field. This can be obtained either by using realistic radiative-hydrodynamic models of the solar photosphere to constrain the radiation field (Trujillo Bueno, Shchukina, and Asensio Ramos, 2004) or by employing differential measures of two or more spectral lines with similar formation properties and varying Hanle sensitivity (e.g. Kleint et al., 2010, 2011). The broad spectral coverage offered by the DKIST/ViSP spectropolarimeter will enable the simultaneous monitoring of multiple lines with differing Hanle sensitivities, contributing to a deeper understanding of the uncertainties in scattering-polarization measurements and allowing the development of multiline scattering-polarization inversion methodologies. The consequent ability to deduce both the strength and direction of the weak fields pervading solar photosphere, and subsequent systematic monitoring of this "turbulent magnetic field" over a solar cycle, will yield evidence as to whether that field is of a local or larger-scale origin.

Outside of the photosphere, pre-DKIST evidence for turbulence has been found in the vicinity of chromospheric shocks that result from the steepening of acoustic waves as they propagate upward from the photosphere (Reardon et al., 2008; De Pontieu et al., 2015). The generation of post-shock turbulence may provide a mechanism for the dispersal of the wave energy beyond the local shock region itself, and may thus be important for chromospheric 
heating. Because the turbulent region is permeated by magnetic field, it may also play a role in wave-mode conversion, coupling the acoustic waves to Alfvénic motions that continue to propagate outward, transmitting energy to higher layers of the solar atmosphere (Reardon et al., 2008). While direct investigation of shock heating by plasma processes at the dissipative scale is out of reach, DKIST may be able to probe the larger-scale properties of the shock region to infer the MHD shock type (e.g. Delmont and Keppens, 2011; Priest, 2014) and the shock-instability processes. A number of shock-instability mechanisms have been identified in other settings: MHD wave breaking (Moore et al., 1987), radiative instability of the shock front (e.g. Smith, 1989; Mignone, 2005), and shock-front distortions due to plasma inhomogeneities (Brouillette, 2002; Zhou, 2017, and the references therein; Markhotok, 2018). In order for any of these to occur in the chromosphere, not only must the instability mechanism be feasible, but it must occur on a time scale short compared to shock-damping rates (Hollweg, 1987; Lanzerotti and Uberoi, 1988). Only limited numerical explorations of shock dynamics within the complex solar atmosphere have been undertaken (Santamaria et al., 2016; Popescu Braileanu et al., 2019a,b). Future idealized studies of shock-instability mechanisms in a magnetized and partially ionized plasma, when constrained by observations at DKIST spatial and temporal resolutions, should lead to a more complete understanding of the physical processes responsible for shock-induced turbulence in the solar chromosphere, and in turn help clarify the underlying plasma processes that result in chromospheric heating and particle acceleration.

Magnetic reconnection is similarly a process that occurs at fundamental scales well below DKIST resolution, but to the understanding of which DKIST can contribute. Observations of macroscale flows associated with a canonical reconnection magnetic-field configuration in a flaring active region support reconnection as the underlying mechanism quite convincingly (Wang et al., 2017b), but such observations are rare, and smaller-scale evidence that can be used to better constrain the physical processes involved are even more elusive. Many theoretical studies, starting with the pioneering work of Forbes and Priest (1983a,b), indicate that magnetic reconnection is enhanced as a result of magnetic-island formation in the plasma current sheet. Magnetic-island formation appears to be ubiquitous (e.g. Loureiro, Schekochihin, and Cowley, 2007; Bhattacharjee et al., 2009; Leake et al., 2012; Mei et al., 2012; Murphy et al., 2012; Ni et al., 2015; Innes et al., 2015; Peter et al., 2019), occurring with or without ion-neutral collisions and with or without a guide field (which tends to reduce the importance of ambipolar diffusion). Further, while the magnetic islands likely have scales well below current and future observational capabilities, bright localized plasmoidlike ejecta have been reported in post coronal mass ejection current sheets in the solar corona (e.g. Riley et al., 2007; Wang, Sui, and Qiu, 2007; Takasao et al., 2012; Guo, Bhattacharjee, and Huang, 2013) and at smaller scales in chromospheric jets and Ellerman-bomb events (e.g. Hu, Song, and Li, 1995; Shibata et al., 2007; Singh et al., 2012; Rouppe van der Voort et al., 2017). With regular observations of such events at DKIST spatial and temporal resolutions, it may be possible to use the statistical properties of these "blobs" to constrain the reconnection magnetic-field and flow configurations and the underlying reconnection processes (e.g. Lin et al., 2007; Lin, Cranmer, and Farrugia, 2008; Song et al., 2012; Guo, Bhattacharjee, and Huang, 2013; Rouppe van der Voort et al., 2017). Further, recent work suggests that, while magnetic-island substructure in the reconnection current sheet is not resolvable, it may be possible to assess the overall complexity of the underlying unresolved field by measuring a reduction in linear polarization (French et al., 2019). 

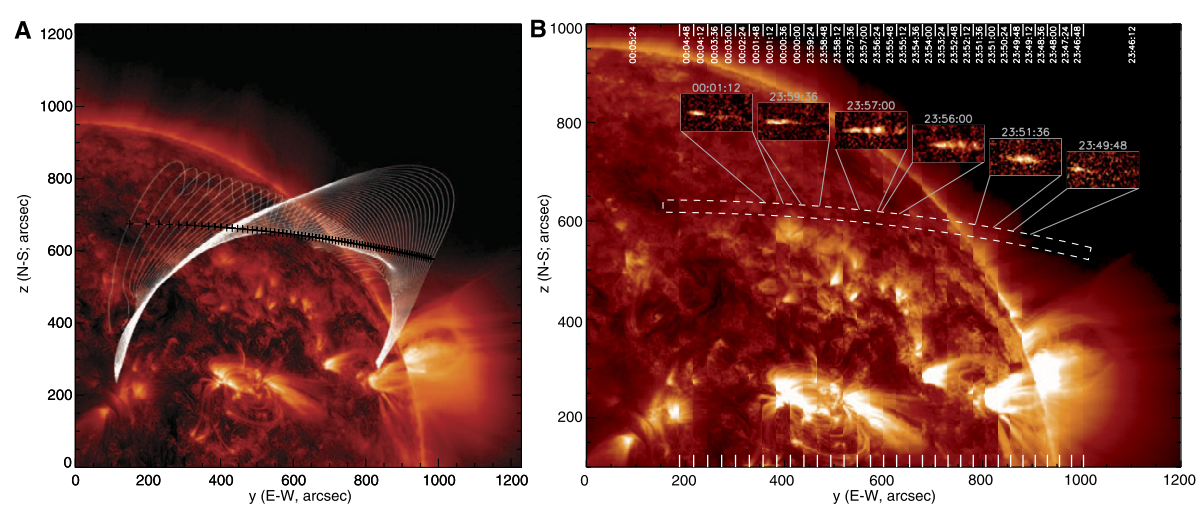

Figure 18 (A) EUV (AIA $17.1 \mathrm{~nm}$ ) image of the solar corona, overlaid in black with the projected orbit of the comet $\mathrm{C} / 2011 \mathrm{~N} 3$. Orbital positions marked by plus signs were used as starting points in a three-dimensional potential-field source-surface extrapolation of the Sun's magnetic field, shown in white. (B) Composite of AIA $17.1 \mathrm{~nm}$ images of the comet moving within the dashed region outlined. The six insets show an enlarged view of the comet at selected times in running-difference images. From Schrijver et al. (2012), copyright by AAAS.

\subsection{Sun-Grazing Comets}

What can we learn from Sun-grazing comets about the solar corona and about cometary composition and structure? Are Sun-grazing comets a significant source of solar-wind pickup ions?

Comets are among the most pristine bodies within the solar system. They provide critical clues about our solar system's formation and the origin of life on Earth. Typically $0.3-5 \mathrm{~km}$ in radius, comets are composed of a mixture of icy, organic, and silicate materials. Sungrazing comets, those with perihelion distances of less than a few solar radii $\left(<3.45 \mathrm{R}_{\odot}\right.$ from the Sun's center, within the fluid Roche limit, Jones et al., 2018), are valuable tools in both cometary and coronal studies. The intense solar radiation that they are subject to during their close perihelion passages evaporates thick layers of near-surface material, exposing their otherwise-invisible pristine interiors, and their high-speed intrusion into the million-degree magnetized solar corona, in extreme cases skimming or plunging into the solar surface (Brown, Carlson, and Toner, 2015), makes them natural probes of regions of the solar atmosphere inaccessible to human-made in-situ instruments. DKIST is well poised to play a unique role in such studies over the coming decades (Raymond et al., 2019).

Over the past two decades, solar space missions have contributed significantly to the study of Sun-grazing comets. The Large Angle and Spectrometric Coronagraph (LASCO), the white-light coronagraph onboard SOHO, has observed more than 3200 Sun-grazing and near-Sun comets, with an average occurrence rate of one every two to three days (Battams and Knight, 2017). A key advance over the past decade has been the detection of Sun-grazing comets, notably comets C/2011 N3 (SOHO) and C/2011 W3 (Lovejoy), in the lower solar corona (Figure 18), a region usually blocked by a coronagraph's occulting disk, using (E)UV instruments onboard SDO, STEREO, and SOHO. Observations of these deeply penetrating comets have provided unique diagnostics of the coronal plasma and magnetic fields (e.g. Schrijver et al., 2012; Raymond et al., 2014; Downs et al., 2013). Ground-based solar telescopes have also observed Sun-grazing comets, including C/2012 
S1 (ISON), which was observed with NSO's Dunn Solar Telescope and McMath-Pierce facility (Wooden et al., 2013) and with the Mees Observatory coronagraph on the summit of Haleakalā, Maui, Hawaii (Druckmüller et al., 2014).

Unlike virtually all other remote-sensing diagnostics of the solar corona, which are subjected to either line-of-sight integration or height ambiguity, Sun-grazing comets take a very localized path through the corona, serving as probes. The comets interact with the plasma producing observable signatures along specific paths through the three-dimensional corona. To date, this has largely been exploited at UV and EUV wavelengths. For example, O III and O VI emission from photo-dissociated cometary water has been used to diagnose the magnetic-field direction and plasma density in the corona (Raymond et al., 2014). Using DKIST's Cryo NIRSP coronographic capabilities, comparable analysis may be possible by observing lines of photo-dissociated silicates such as Si IX. Similarly, observations of Lyman- $\alpha$ emission during passage of Sun-grazing comets has been used to estimate the coronal density, temperature, and solar-wind velocity (Bemporad et al., 2015). The same techniques may be possible with DKIST using $\mathrm{H} \alpha$ or Paschen- $\alpha$ emission. Moreover, ion tails have been seen accompanying a few Sun-grazing comets in white light (Jones et al., 2018), and their presence in exocomets is inferred from their Ca II absorption signatures (Kiefer et al., 2014). Observations of comet-tail-heliospheric current-sheet interactions during cometary crossings may provide constraints on the current-sheet morphology and the solar-wind structure in the inner heliosphere (see Jones et al., 2018, and the references therein). Such observations by DKIST would be groundbreaking.

Beyond diagnostics of the solar corona, DKIST promises to contribute directly to the cometary science. Most fundamentally, it will enable measurements of the size and composition of cometary cores (Bryans and Pesnell, 2016) as they are exposed very close to the Sun, too close for observation using night-time telescopes. While some Sun-grazing comets, such as Lovejoy and ISON, are discovered at great distances from the Sun and followed to their perihelia, many are not active enough to be observed at large distances and are first noticed close in to the Sun, within the field of view of LASCO. Close to the Sun, cometary material sublimates rapidly and is photo-dissociated to form atomic species, which are then ionized through successive ionization states (Bryans and Pesnell, 2012; McCauley et al., 2013), with optical and infrared line emission largely confined to a small region surrounding the cometary nucleus. The time-dependent emission signatures allow characterization of both the cometary material and the local-coronal plasma environment. DKIST's unique capabilities will make these otherwise very difficult spectrographic measurements of pristine cometary material possible. Moreover, DKIST infrared observations will allow measurements of cometary dust temperatures and dust sublimation rates. These are particularly important in advancing our understanding of the origin of cometary neutral tails (e.g. Cremonese et al., 2002), and they may be critical in determining the inner source of pickup ions in the solar wind (e.g. Bzowski and Królikowska, 2005). Finally, previous attempts to determine the tensile strength (if any) of Sun-grazing comets from their breakup (Opik, 1966; Klinger, Espinasse, and Schmidt, 1989) have been inconclusive. This is an important measurement in the context of planetesimal formation, and DKIST will bring much higher spatial resolution to bear on the assessment of cometary-fragmentation processes.

\subsection{Mercury Transit Science}

What can we learn about Mercury's atmosphere and its seasonal variations from DKIST observations of Mercury's transit across the Sun? Can the resonance absorption lines of $K$ and Ca be observed at DKIST sensitivities? 
Figure 19 Sodium absorption equivalent width around the disk of Mercury during the 8

November 2006 transit. The noise outside the close region of sodium absorption is due to fluctuations in the solar intensity. The strongest atmospheric absorption is found over Mercury's north and south poles. Likely due to seasonal variations, the dawn/dusk terminator difference identified in the previous transit (Schleicher et al., 2004) is not seen during this one. From Potter et al. (2013), copyright by Elsevier.

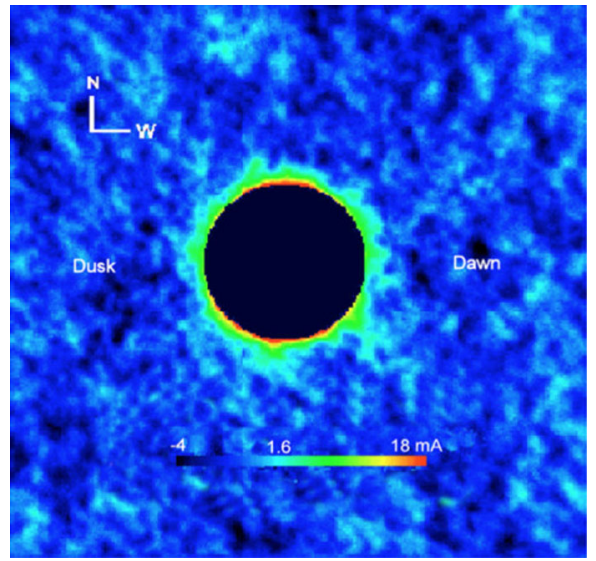

There was some hope that early DKIST observations might overlap with the 11 November 2019 Mercury transit. Unfortunately, it was not possible to meet that aggressive goal, which lay outside of the nominal DKIST construction timeline. We include a brief description of the science goals of Mercury-transit observations here to illustrate the scientific flexibility of the DKIST observing system and to point to future possibilities within the DKIST lifetime. The next partial Mercury transits visible from Haleakalā are one ending in the early morning of 7 May 2049 and one occurring midday to sunset on 8 November 2052. (eclipse. gsfc.nasa.gov/transit/catalog/MercuryCatalog.html, www.timeanddate.com/eclipse/in/usa).

Mercury has a non-spherical, seasonally varying exosphere (e.g. Domingue et al., 2007; McClintock et al., 2008; Cassidy et al., 2015; Vervack et al., 2016; Merkel et al., 2017) and a dynamic magnetosphere with dayside reconnection and magnetotail activity resembling that found at Earth (Slavin et al., 2009, 2010; Bagenal, 2013). Full characterization of this unique atmosphere is challenging. Traditional remote-sensing techniques are difficult due to the Sun's proximity, and downlink limitations led to NASA's Mercury Surface, Space Environment, Geochemistry, and Ranging (MESSENGER) orbiter carrying a single-point rather than slit-scanning spectrometer. To date, transit spectroscopy (observations of absorption by Mercury's atmospheric constituents during rare solar transits) has offered some of the highest spatial, temporal, and spectral resolution measurements of Mercury's atmosphere (Figure 19; Schleicher et al., 2004; Potter et al., 2013; Schmidt et al., 2018). So far only sodium in Mercury's atmosphere has been measured during transit, but with DKIST's sensitivity, observations of the $\mathrm{K}$ and $\mathrm{Ca}$ atomic resonance absorption lines may be possible. The distribution of these in the Mercury atmosphere would constrain source and loss processes (Burger et al., 2012). Broad-band absorption measurements would additionally contribute to our understanding of the dust density distribution around the planet.

The scientific use of Mercury-transit measurements has progressed rapidly despite the rarity of transit events. Measurements of $\mathrm{Na}$ I absorption by transit spectroscopy were first made in the early years of this century (Schleicher et al., 2004). These provided convincing evidence for a dawn-side enhancement in Mercury's exosphere. Subsequent measurements by Potter et al. (2013) were markedly different, showing no dawn/dusk terminator difference (Figure 19). That is now understood to be the result of seasonal variations. Seasonal variations were identified in MESSENGER orbiter data (Merkel et al., 2017), and confirmed by later transit observations (Schmidt et al., 2018). The 2018 transit measurements indicate nearly identical $\mathrm{Na}$ distribution to that obtained from the 2004 observations taken during the same Mercury season. The quality of the 2018 observations allowed spatial resolution 
of the Mercury atmospheric scale height $(\approx 100 \mathrm{~km})$ and enabled study of exospheric timedependence induced by solar-wind interactions. During future transits, DKIST capabilities will enable spectral analysis of Doppler velocities, $\approx 90 \mathrm{~km}$ resolution of the atmospheric stratification, and temporal resolution of solar-wind and interplanetary magnetic-field angle influences.

\subsection{Synergistic Opportunities with In-Situ Measurements}

How well can the magnetic connectivity between the Sun and the in-situ measurements of Parker Solar Probe and Solar Orbiter be established? Which aspects of the in-situ measurements originate at the Sun and which result from subsequent dynamics and instabilities in the solar wind?

DKIST, Parker Solar Probe, and Solar Orbiter will together allow unprecedented synergistic study of the connectivity between the solar corona and the inner heliosphere. Parker Solar Probe and Solar Orbiter will make in-situ measurements of the inner heliospheric electric and magnetic fields and plasma kinetic properties, while DKIST and Solar Orbiter will image and make high-precision spectropolarimetric measurements of the solar atmosphere from the deep photosphere to the corona. The combined capabilities of these assets will revolutionize our understanding of how stars create and control their magnetic environments (Martinez Pillet et al., 2020; Müller et al., 2020; Velli et al., 2020).

Parker Solar Probe's close approaches to the Sun and Solar Orbiter's inclined orbit with perihelia inside the orbit of Mercury will allow direct sampling of the solar-wind plasma before it has undergone extensive evolution and mixing. The plasma sampled will preserve many of the signatures of its origins, allowing assessment of the acceleration mechanisms underlying different solar-wind components. Moreover, the proximity of Parker Solar Probe's perihelia to the Sun allows periods of co-rotation with the solar surface and more importantly with the magnetic field that originates there. This will enable studies of the relationship between temporal variations in the wind and short-term changes at the source. Separating these from the variations due to the spacecraft motion across solar-wind structures is critical to understanding both the source behavior and the secondary development of the wind itself. Similarly, periods of quasi-co-rotation by Solar Orbiter will enable extended observations of the same solar region (Müller et al., 2013). These periods will help connect solar-activity evolution to changes in the wind over somewhat longer time scales. Finally, Parker Solar Probe will spend about 15 hours below ten solar radii, during which it will likely sample the sub-Alfvénic solar wind, allowing direct assessment of the conditions under which solar-wind heating and acceleration likely occur (e.g. Cranmer and van Ballegooijen, 2005).

These scientific goals all require, or strongly benefit from, knowing how the regions of in-situ heliospheric measurements are connected to the magnetic-field and particle source regions in the solar atmosphere. An example mapping is shown in Figure 20. It assumes a radial solar wind of constant speed between PSP and $15 \mathrm{R}_{\odot}$, and it uses the Current Sheet Source Surface model (CSSS, developed by Zhao and Hoeksema, 1995, see also Poduval and Zhao, 2014, Poduval, 2016, Chandorkar et al., 2020) to determine the corresponding photospheric foot points. Model improvements above the source surface, employing solarwind measurements from the PSP Solar Wind Electrons Alphas and Protons (SWEAP) suite of instruments, are anticipated as those data become publicly available (Poduval et al., 2021), and DKIST's ability to quantitatively measure the magnetic field in the solar chromosphere and low solar corona will significantly improve the ability to map the connectivity from there 


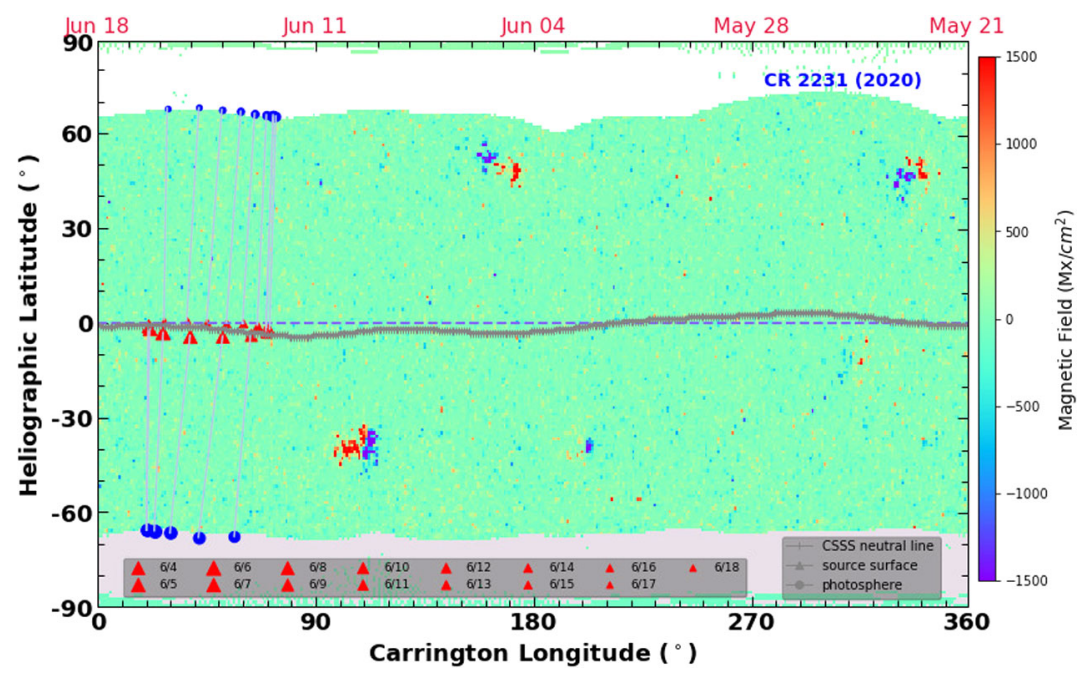

Figure 20 The locations of Parker Solar Probe (4 - 18 June 2020), mapped back to the Sun using the Current Sheet Source Surface model (CSSS, developed by Zhao and Hoeksema, 1995, see also Poduval and Zhao, 2014, Poduval, 2016, Chandorkar et al., 2020), superimposed on an SDO/HMI synoptic magnetogram (Carrington rotation 2231, downsampled to one-degree resolution). The red triangles are the map-back locations at $15 \mathrm{R}_{\odot}$ (the CSSS source surface) assuming a constant radial solar-wind speed of $395 \mathrm{~km} \mathrm{~s}^{-1}$. These are joined to their photospheric foot points (blue filled circles) by thin gray lines to indicate connectivity. The CSSS deduced open magnetic-field regions (coronal holes) are shown in white and gray in northern and southern hemispheres, respectively, with the magnetic polarity inversion line (heliospheric current sheet) marked with dark-gray plus symbols. Figure courtesy of B. Poduval.

to source regions on the Sun (Sections 5.2, 5.6). Both field extrapolation (e.g. Badman et al., 2020) and magnetohydrodynamic modeling methods (van der Holst et al., 2019; Riley et al., 2019), which currently employ moderate-resolution synoptic photospheric magnetograms, will significantly benefit from high-resolution multi-height DKIST data.

Moreover, the chemical composition of the solar wind is a key indicator of its origin (e.g. Geiss, Gloeckler, and von Steiger, 1995; Parenti et al., 2000; Brooks and Warren, 2011; Brooks, Ugarte-Urra, and Warren, 2015; Baker et al., 2015), and the first ionization potential (FIP) bias can be used to help trace that origin and establish magnetic connectivity with in-situ measurements (Section 5.1). There is evidence that active regions emerge with photospheric abundances and develop a FIP bias in the chromosphere, which then propagates into the corona (Laming, 2015). This is supported by an observed lag between magneticactivity indices and abundance fluctuations in the solar wind at $1 \mathrm{AU}$ (Wind spacecraft at $\mathrm{L}_{1}$; Alterman and Kasper, 2019). With the synergistic capabilities of DKIST and Solar Orbiter, the underlying causes for these correlations can be more directly examined. In-situ instrumentation on Solar Orbiter is designed to measure abundance ratios, and the FIP bias measured in situ combined with chromospheric observations using DKIST can be used to more precisely assess the plasma's origin.

Similarly, there is a relationship between in-situ measurements of helium at $1 \mathrm{AU}$, solar activity, and the solar-wind speed (Aellig, Lazarus, and Steinberg, 2001; Kasper et al., 2007, 2012; Alterman and Kasper, 2019). The lowest helium abundances are observed during solar minimum and are correlated with regions of slower wind speed. The helium abundance in the fast solar wind changes little with solar cycle. The mechanisms underlying these correlations are unknown, but the combination of in-situ measurements and DKIST's unique 
capabilities will, again, allow new ways to investigate this fundamental problem. Previous observations indicate that helium depletion likely occurs below the solar corona (Laming and Feldman, 2001, 2003), and models suggest that the process is sensitive to the partially ionized state of the chromospheric plasma (Laming, 2015). Parker Solar Probe will make helium-abundance measurements close to the Sun, reducing uncertainties in source-region identification. DKIST will not only assist with that mapping but enable key chromospheric observations to address the underlying helium-depletion processes.

The dust content of the inner solar corona is also a question of significant importance. It has practical importance both for the survival of the Parker Solar Probe and for the success of coronal spectropolarimetric measurements using DKIST. Observing the white light scattered by dust (F-corona) and electrons (Thomson scattering), the Wide-field Imager for Parker Solar Probe (WISPR) images the large-scale structure of the corona before the spacecraft passes through it. As the orbit perihelion is reduced, WISPR will help determine whether a dust-free zone exists near the Sun. Moreover, during the innermost perihelion passage of 2024, the boundary of the WISPR field-of-view will extend down to two solar radii above the solar photosphere, close to the outermost height that will be observable by DKIST $\left(1.5 \mathrm{R}_{\odot}, 0.5\right.$ solar radius above the limb). This proximity of the fields of view will provide a unique opportunity to test whether the diffuse coronal He I $1083 \mathrm{~nm}$ brightness reported by Kuhn, Penn, and Mann (1996) and Kuhn et al. (2007) can be accounted for by helium neutralization on the surface of dust particles within the hot corona, as proposed by Moise, Raymond, and Kuhn (2010). That hypothesis has been difficult to test, but it is important because the He I $1083 \mathrm{~nm}$ line is the only permitted infrared transition available to DKIST for spectropolarimetric observations of the corona.

If the neutral-helium signal indeed originates within the corona, new coronal Hanle magnetic-field diagnostics are possible (Dima, Kuhn, and Berdyugina, 2016). Combined with linear-polarization measurements in the SiX $1430 \mathrm{~nm}$ forbidden line, which is in the saturated Hanle regime under coronal conditions, polarization observations of the He I $1083 \mathrm{~nm}$ permitted line would enable inference of all three components of the coronal magnetic field. This would critically constrain the coronal magnetic topology, and in turn would allow improvements in the accuracy of the coronal-heliospheric models used to predict the heliospheric magnetic configuration. Such improvements are particularly important during periods in which the Sun is more active and the corona is consequently more complex than it is currently (e.g. Raouafi et al., 2016), periods likely to be encountered during the later perihelia of the Parker Solar Probe mission. Given the uncertainties, the lines and techniques employed by DKIST's synoptic effort (Section 6.1 above) to regularly measure the solar coronal magnetic fields in anticipation of Parker Solar Probe encounters will likely evolve in order to generate the best input data for coronal models. Along with the anticipated Si X $1430 \mathrm{~nm} / \mathrm{He}$ I $1083 \mathrm{~nm}$ observations motivated above, early observations will include the forbidden Fe XIII line at $1075 \mathrm{~nm}$, as the current High Altitude Observatory's Coronal Multi-channel Polarimeter (HAO/CoMP), but with the capability of regularly measuring the Stokes $-V$ component. That component, accessible to DKIST because of its anticipated polarization sensitivity, will allow robust inference of the line-of-sight magnetic field.

Another important problem, the solution to which the synergistic capabilities of DKIST, Parker Solar Probe, and Solar Orbiter may significantly contribute, is the so-called openflux problem (Linker et al., 2017; Riley et al., 2019). A significant portion of the magnetic field at the solar surface opens out into the heliosphere, forming coronal holes. This occurs where facular fields of a dominant polarity cover a large area, usually at the polar caps but also sometimes at low latitudes, and the solar-wind pressure is sufficient to open much of the field at height. The open-flux problem describes the disparity between the total open 
magnetic flux at the Sun, as estimated from moderate-resolution magnetograms of coronalhole regions (defined as regions dark in EUV and x-ray emission), and the total open flux measured in situ at 1 AU (Linker et al., 2017). The former value falls significantly below the latter. This discrepancy is critical to solar-wind and heliospheric modeling, which relies on the solar value, as determined from surface observations, for a boundary condition. The mismatch in measured values implies either that a significant amount of open flux at the Sun lies below the sensitivity of current instrumentation, that the polar magnetic flux for example is significantly underestimated due to difficulties observing it (Riley et al., 2019), or that the open flux measured at 1 AU does not map exclusively to coronal holes. The later possibility relates the open-flux problem to that of the origin of the slow solar wind, supporting suggestions that the slow solar wind arises from mixed open- and closed-field regions, possibly at the coronal-hole boundaries (e.g. Linker et al., 2017; Owens et al., 2020, and the references therein). However, it is currently uncertain what the true magnitude of the open-flux problem is, whether it persists if the in-situ measurements are made in the inner heliosphere, or whether its origin lies with field reconfiguration in the solar wind inward of 1 AU. Parker Solar Probe and Solar Orbiter magnetic field measurements will address the in-situ uncertainties directly, within the limitations of single-point measurements (Owens et al., 2008, and the references therein), and observations with the spatial resolution and polarization sensitivity available to DKIST will reveal the amount of small-scale open flux that currently remains undetected in less, sensitive full-disk solar magnetograms. Together these will either reconcile the in-situ and remote-sensing open-flux deductions or elucidate the magnitude and perhaps the source of the discrepancy.

Finally, one of the most prominent early results of the Parker Solar Probe mission has been observations of magnetic switchbacks (Bale et al., 2019; Kasper et al., 2019; Dudok de Wit et al., 2020; Horbury et al., 2020; Mozer et al., 2020): rapid changes (over intervals ranging from seconds to tens of minutes, perhaps hours) in the radial magnetic-field orientation, away from and then back to its original orientation, with field deflections sometimes amounting to a full reversal with respect to the Parker spiral. Such field switchbacks are accompanied by rapid enhancement of the radial wind speed, and they are often called velocity spikes for that reason. The correlation between the magnetic-field perturbations and jet-like flows suggests that these events are large-amplitude Alfvénic structures being advected away from the Sun by the solar wind (Bale et al., 2019). Their origin remains uncertain. Clustering and correlation statistics of their occurrence during the first Parker Solar Probe perihelion encounter (a ten-day period, centered on perihelion at $\approx 36 \mathrm{R}_{\odot}$ ) suggests that they are remnants of stronger events in the low corona (Dudok de Wit et al., 2020), but comparison between perihelion (one-day period at $\approx 36 \mathrm{R}_{\odot}$ ) and pre-perihelion (oneday period five days earlier at $\approx 48 \mathrm{R}_{\odot}$ ) intervals during the second encounter suggests that the number of switchbacks and the magnetic-field rotation angle of the field within the switchbacks increases with increasing distance from the Sun (Mozer et al., 2020). Theoretical work is also inconclusive, indicating that switchback-like structures that originate in the lower solar corona can indeed survive in the solar wind out to Parker Solar Probe distances (Tenerani et al., 2020), but also that such structures can form in the expanding solar wind itself as growing Alfvénic fluctuations (Squire, Chandran, and Meyrand, 2020).

Sudden reversals of the magnetic field and associated jet-like flows are not new to Parker Solar Probe. They have been observed over several decades in earlier in-situ measurements (see references within Horbury et al., 2020; Dudok de Wit et al., 2020). What separates the Parker Solar Probe observations from those earlier measurements is the occurrence of switchbacks in relatively slow solar-wind environments and the large number and magnitude of the events encountered. Thousands of such events have been observed by the Parker 
Solar Probe, some in tight clusters separated by relatively event-free regions. This clustering, the significant correlation between the magnetic-field deflection observed for individual events in a cluster, and the similarity of the flows observed to those deduced for other coronal jets all support a low coronal origin (Horbury et al., 2020). This has led to the suggestion that they are folds in the magnetic field that originate as ubiquitous interchange reconnection events lower down, possibly driven by the global circulation of open flux at the Sun (Fisk and Kasper, 2020). DKIST's chromospheric and low-corona observing capabilities will contribute to the assessment of the origin of switchbacks, both by improving the determinations of the connectivity to the source regions, as discussed earlier, and by directly looking for evidence of interchange reconnection heating or jets in the source regions.

\section{Conclusion}

The science to which DKIST observations will contribute is wide ranging, from fundamental physics with technological and astrophysical applications to solar and heliospheric physics with terrestrial and exoplanet habitability implications. We have tried to outline the compelling range of possibilities here, not to restrict them but to celebrate the vista that has opened up with the advent of the National Science Foundation's four meter Daniel K. Inouye Solar Telescope. The order in which this exhilarating array of scientific objectives will be addressed will be determined by the scientific community itself, as access to DKIST will be observing-proposal based. With progress, the snapshot we presented here will age, and new compelling scientific goals will emerge. The DKIST has been designed and constructed to flexibly accommodate those for many years to come.

Acknowledgements This work rests on many years of sustained vision, effort, and dedication by DKIST and DKIST instrument, team scientists, engineers, and administrative, support personnel, the unwavering commitment of the National Science Foundation, and the support of the US taxpayers. It includes contributions from members of the DKIST Science Working Group and the DKIST Critical Science Plan Community, all of whom generously shared their experiences, plans, knowledge, and dreams. It is impossible at this stage to fully acknowledge each individual's contribution to those groups without error or error of omission, but they are all greatly appreciated.

The research reported herein was based in part on the National Science Foundation's Daniel K. Inouye Solar Telescope (DKIST), a facility of the National Solar Observatory (NSO). NSO is managed by the Association of Universities for Research in Astronomy, Inc., under a cooperative agreement with the National Science Foundation. Any opinions, findings, and conclusions or recommendations expressed in this publication are those of the authors and do not necessarily reflect the views of the National Science Foundation or the Association of Universities for Research in Astronomy, Inc.

DKIST is located on land of spiritual and cultural significance to Native Hawaiian people. The use of this important site to further scientific knowledge is done with great appreciation and respect.

Funding Open Access funding provided by the National Solar Observatory (NSO). The NSO is operated by the Association of Universities for Research in Astronomy, Inc., and is funded by the National Science Foundation.

Disclosure of Potential Conflicts of Interest The authors declare that they have no conflicts of interest.

Publisher's Note Springer Nature remains neutral with regard to jurisdictional claims in published maps and institutional affiliations.

Open Access This article is licensed under a Creative Commons Attribution 4.0 International License, which permits use, sharing, adaptation, distribution and reproduction in any medium or format, as long as you give appropriate credit to the original author(s) and the source, provide a link to the Creative Commons 
licence, and indicate if changes were made. The images or other third party material in this article are included in the article's Creative Commons licence, unless indicated otherwise in a credit line to the material. If material is not included in the article's Creative Commons licence and your intended use is not permitted by statutory regulation or exceeds the permitted use, you will need to obtain permission directly from the copyright holder. To view a copy of this licence, visit http://creativecommons.org/licenses/by/4.0/.

\section{References}

Abramenko, V.I., Carbone, V., Yurchyshyn, V., Goode, P.R., Stein, R.F., Lepreti, F., Capparelli, V., Vecchio, A.: 2011, Turbulent diffusion in the photosphere as derived from photospheric bright point motion. Astrophys. J. 743, 133. DOI. ADS.

Aellig, M.R., Lazarus, A.J., Steinberg, J.T.: 2001, The solar wind helium abundance: variation with wind speed and the solar cycle. Geophys. Res. Lett. 28, 2767. DOI. ADS.

Agrawal, P., Rast, M.P., Gošić, M., Bellot Rubio, L.R., Rempel, M.: 2018, Transport of internetwork magnetic flux elements in the solar photosphere. Astrophys. J. 854, 118. DOI. ADS.

Aiouaz, T., Rast, M.P.: 2006, Expansion of the supergranular magnetic network through the solar atmosphere. Astrophys. J. Lett. 647, L183. DOI. ADS.

Aiouaz, T., Peter, H., Lemaire, P.: 2005, The correlation between coronal Doppler shifts and the supergranular network. Astron. Astrophys. 435, 713. DOI. ADS.

Alaoui, M., Holman, G.D.: 2017, Understanding breaks in flare X-ray spectra: evaluation of a cospatial collisional return-current model. Astrophys. J. 851, 78. DOI. ADS.

Alazraki, G., Couturier, P.: 1971, Solar wind accejeration caused by the gradient of Alfven wave pressure. Astron. Astrophys. 13, 380. ADS.

Alexakis, A., Mininni, P.D., Pouquet, A.: 2006, On the inverse cascade of magnetic helicity. Astrophys. J. 640, 335. DOI. ADS.

Allred, J.C., Hawley, S.L., Abbett, W.P., Carlsson, M.: 2005, Radiative hydrodynamic models of the optical and ultraviolet emission from solar flares. Astrophys. J. 630, 573. DOI. ADS.

Alpert, S.E., Tiwari, S.K., Moore, R.L., Winebarger, A.R., Savage, S.L.: 2016, Hi-C observations of sunspot penumbral bright dots. Astrophys. J. 822, 35. DOI. ADS.

Alterman, B.L., Kasper, J.C.: 2019, Helium variation across two solar cycles reveals a speed-dependent phase lag. Astrophys. J. Lett. 879, L6. DOI. ADS.

Amari, T., Luciani, J.-F., Aly, J.-J.: 2015, Small-scale dynamo magnetism as the driver for heating the solar atmosphere. Nature 522, 188. DOI. ADS.

Amari, T., Luciani, J.F., Aly, J.J., Mikic, Z., Linker, J.: 2003, Coronal mass ejection: initiation, magnetic helicity, and flux ropes. I. Boundary motion-driven evolution. Astrophys. J. 585, 1073. DOI. ADS.

Amari, T., Aly, J.-J., Mikic, Z., Linker, J.: 2010, Coronal mass ejection initiation: on the nature of the flux cancellation model. Astrophys. J. Lett. 717, L26. DOI. ADS.

Ambastha, A., Basu, S., Antia, H.M.: 2003, Flare-induced excitation of solar p modes. Solar Phys. $218,151$. DOI. ADS.

Anan, T., Casini, R., Ichimoto, K.: 2014, Diagnosis of magnetic and electric fields of chromospheric jets through spectropolarimetric observations of H I Paschen lines. Astrophys. J. 786, 94. DOI. ADS.

Anan, T., Ichimoto, K., Hillier, A.: 2017, Differences between Doppler velocities of ions and neutral atoms in a solar prominence. Astron. Astrophys. 601, A103. DOI. ADS.

Anderson, L.S., Athay, R.G.: 1989, Chromospheric and coronal heating. Astrophys. J. 336, 1089. DOI. ADS.

Antiochos, S.K., DeVore, C.R., Klimchuk, J.A.: 1999, A model for solar coronal mass ejections. Astrophys. J. 510, 485. DOI. ADS.

Antolin, P., Rouppe van der Voort, L.: 2012, Observing the fine structure of loops through high-resolution spectroscopic observations of coronal rain with the CRISP instrument at the Swedish Solar Telescope. Astrophys. J. 745, 152. DOI. ADS.

Antolin, P., Shibata, K., Vissers, G.: 2010, Coronal rain as a marker for coronal heating mechanisms. Astrophys. J. 716, 154. DOI. ADS.

Antolin, P., Okamoto, T.J., De Pontieu, B., Uitenbroek, H., Van Doorsselaere, T., Yokoyama, T.: 2015a, Resonant absorption of transverse oscillations and associated heating in a solar prominence. II. Numerical aspects. Astrophys. J. 809, 72. DOI. ADS.

Antolin, P., Vissers, G., Pereira, T.M.D., Rouppe van der Voort, L., Scullion, E.: 2015b, The multithermal and multi-stranded nature of coronal rain. Astrophys. J. 806, 81. DOI. ADS.

Apai, D., Rackham, B.V., Giampapa, M.S., Angerhausen, D., Teske, J., Barstow, J., Carone, L., Cegla, H., Domagal-Goldman, S.D., Espinoza, N., Giles, H., Gully-Santiago, M., Haywood, R., Hu, R., Jordan, A., Kreidberg, L., Line, M., Llama, J., López-Morales, M., Marley, M.S., de Wit, J.: 2018, Understanding 
stellar contamination in exoplanet transmission spectra as an essential step in small planet characterization. ADS. arXiv.

Archontis, V., Hood, A.W.: 2009, Formation of Ellerman bombs due to 3D flux emergence. Astron. Astrophys. 508, 1469. DOI. ADS.

Armitage, P.J.: 2019, Physical processes in protoplanetary disks. In: Audard, M., Meyer, M.R., Alibert, Y. (eds.) From Protoplanetary Disks to Planet Formation, Saas-Fee Advanced Course 45, Springer, Heidelberg, 1. DOI. ADS.

Aschwanden, M.J.: 2001, An evaluation of coronal heating models for active regions based on Yohkoh, SOHO, and TRACE observations. Astrophys. J. 560, 1035. DOI. ADS.

Aschwanden, M.J.: 2006, Coronal magnetohydrodynamic waves and oscillations: observations and quests. Phil. Trans. Roy. Soc. London Ser. A 364, 417. DOI. ADS.

Asensio Ramos, A., de la Cruz Rodríguez, J., Martínez González, M.J., Socas-Navarro, H.: 2017, Inference of the chromospheric magnetic field orientation in the Ca II $8542 \AA$ line fibrils. Astron. Astrophys. 599, A133. DOI. ADS.

Asgari-Targhi, M., van Ballegooijen, A.A., Cranmer, S.R., DeLuca, E.E.: 2013, The spatial and temporal dependence of coronal heating by Alfvén wave turbulence. Astrophys. J. 773, 111. DOI. ADS.

Asplund, M., Nordlund, Å., Trampedach, R., Allende Prieto, C., Stein, R.F.: 2000, Line formation in solar granulation. I. Fe line shapes, shifts and asymmetries. Astron. Astrophys. 359, 729. ADS.

Avallone, E.A., Tiwari, S.K., Panesar, N.K., Moore, R.L., Winebarger, A.: 2018, Critical magnetic field strengths for solar coronal plumes in quiet regions and coronal holes? Astrophys. J. 861, 111. DOI. ADS.

Badman, S.T., Bale, S.D., Martínez Oliveros, J.C., Panasenco, O., Velli, M., Stansby, D., Buitrago-Casas, J.C., Réville, V., Bonnell, J.W., Case, A.W., Dudok de Wit, T., Goetz, K., Harvey, P.R., Kasper, J.C., Korreck, K.E., Larson, D.E., Livi, R., MacDowall, R.J., Malaspina, D.M., Pulupa, M., Stevens, M.L., Whittlesey, P.L.: 2020, Magnetic connectivity of the ecliptic plane within 0.5 au: potential field source surface modeling of the first Parker solar probe encounter. Astrophys. J. Suppl. Ser. 246, 23. DOI. ADS.

Bagenal, F.: 2013, Planetary magnetospheres. In: Oswalt, T.D., French, L.M., Kalas, P. (eds.) Planets, Stars and Stellar Systems. Volume 3: Solar and Stellar Planetary Systems, Springer, Dordrecht, 251. DOI. ADS.

Bahauddin, S.M., Rast, M.P.: 2021, Identifying acoustic wave sources on the Sun. I. Two-dimensional waves in a simulated photosphere. Astrophys. J. (submitted). ADS. arXiv.

Bahauddin, S.M., Bradshaw, S.J., Winebarger, A.R.: 2020, The origin of reconnection-mediated transient brightenings in the solar transition region. Nat. Astron. DOI. ADS.

Baker, D., Brooks, D.H., Démoulin, P., Yardley, S.L., van Driel-Gesztelyi, L., Long, D.M., Green, L.M.: 2015, FIP bias evolution in a decaying active region. Astrophys. J. 802, 104. DOI. ADS.

Bale, S.D., Badman, S.T., Bonnell, J.W., Bowen, T.A., Burgess, D., Case, A.W., Cattell, C.A., Chandran, B.D.G., Chaston, C.C., Chen, C.H.K., Drake, J.F., de Wit, T.D., Eastwood, J.P., Ergun, R.E., Farrell, W.M., Fong, C., Goetz, K., Goldstein, M., Goodrich, K.A., Harvey, P.R., Horbury, T.S., Howes, G.G., Kasper, J.C., Kellogg, P.J., Klimchuk, J.A., Korreck, K.E., Krasnoselskikh, V.V., Krucker, S., Laker, R., Larson, D.E., MacDowall, R.J., Maksimovic, M., Malaspina, D.M., Martinez-Oliveros, J., McComas, D.J., Meyer-Vernet, N., Moncuquet, M., Mozer, F.S., Phan, T.D., Pulupa, M., Raouafi, N.E., Salem, C., Stansby, D., Stevens, M., Szabo, A., Velli, M., Woolley, T., Wygant, J.R.: 2019, Highly structured slow solar wind emerging from an equatorial coronal hole. Nature 576, 237. DOI. ADS.

Balmforth, N.J.: 1992a, Solar pulsational stability - I. Pulsation-mode thermodynamics. Mon. Not. Roy. Astron. Soc. 255, 603. DOI. ADS.

Balmforth, N.J.: 1992b, Solar pulsational stability - III. Acoustical excitation by turbulent convection. Mon. Not. Roy. Astron. Soc. 255, 639. DOI. ADS.

Banerjee, D., Erdélyi, R., Oliver, R., O'Shea, E.: 2007, Present and future observing trends in atmospheric magnetoseismology. Solar Phys. 246, 3. DOI. ADS.

Bard, S., Carlsson, M.: 2010, Radiative hydrodynamic simulations of acoustic waves in sunspots. Astrophys. J. 722, 888. DOI. ADS.

Battams, K., Knight, M.M.: 2017, SOHO comets: 20 years and 3000 objects later. Phil. Trans. Roy. Soc. London Ser. A 375, 20160257. DOI. ADS.

Ba̧k-Stȩślicka, U., Gibson, S.E., Fan, Y., Bethge, C., Forland, B., Rachmeler, L.A.: 2013, The magnetic structure of solar prominence cavities: new observational signature revealed by coronal magnetometry. Astrophys. J. Lett. 770, L28. DOI. ADS.

Beaudoin, P., Simard, C., Cossette, J.-F., Charbonneau, P.: 2016, Double dynamo signatures in a global MHD simulation and mean-field dynamos. Astrophys. J. 826, 138. DOI. ADS.

Beckers, J.M.: 1968, Solar spicules (invited review paper). Solar Phys. 3, 367. DOI. ADS.

Bello González, N., Franz, M., Martínez Pillet, V., Bonet, J.A., Solanki, S.K., del Toro Iniesta, J.C., Schmidt, W., Gandorfer, A., Domingo, V., Barthol, P., Berkefeld, T., Knölker, M.: 2010, Detection of large acoustic energy flux in the solar atmosphere. Astrophys. J. Lett. 723, L134. DOI. ADS. 
Bellot Rubio, L., Orozco Suárez, D.: 2019, Quiet Sun magnetic fields: an observational view. Liv. Rev. Solar Phys. 16, 1. DOI. ADS.

Bemporad, A., Giordano, S., Raymond, J.C., Knight, M.M.: 2015, Study of sungrazing comets with spacebased coronagraphs: new possibilities offered by METIS on board Solar Orbiter. Adv. Space Res. 56, 2288. DOI. ADS.

Benz, A.O., Battaglia, M., Güdel, M.: 2017, Observations of a radio-quiet solar preflare. Solar Phys. 292, 151. DOI. ADS

Berger, M.A.: 1984, Rigorous new limits on magnetic helicity dissipation in the solar corona. Geophys. Astrophys. Fluid Dyn. 30, 79. DOI. ADS.

Berger, M.A.: 1999, Introduction to magnetic helicity. Plasma Phys. Control. Fusion 41, B167. DOI. ADS.

Berger, M.A., Asgari-Targhi, M.: 2009, Self-organized braiding and the structure of coronal loops. Astrophys. J. 705, 347. DOI. ADS.

Bharti, L., Hirzberger, J., Solanki, S.K.: 2013, Fine structures in the atmosphere above a sunspot umbra. Astron. Astrophys. 552, L1. DOI. ADS.

Bhattacharjee, A., Huang, Y.-M., Yang, H., Rogers, B.: 2009, Fast reconnection in high-Lundquist-number plasmas due to the plasmoid instability. Phys. Plasmas 16, 112102. DOI. ADS.

Bhowmik, P., Nandy, D.: 2018, Prediction of the strength and timing of sunspot cycle 25 reveal decadal-scale space environmental conditions. Nat. Comm. 9, 5209. DOI. ADS.

Biermann, L.: 1946, Zur Deutung der chromosphärischen Turbulenz und des Exzesses der UV-Strahlung der Sonne. Naturwissenschaften 33, 118. DOI. ADS.

Birch, A.C., Kosovichev, A.G., Duvall, T.L. Jr.: 2004, Sensitivity of acoustic wave travel times to sound-speed perturbations in the solar interior. Astrophys. J. 608, 580. DOI. ADS.

Blackman, E.G.: 2015, Magnetic helicity and large scale magnetic fields: a primer. Space Sci. Rev. $188,59$. DOI. ADS.

Bloomfield, D.S., McAteer, R.T.J., Mathioudakis, M., Williams, D.R., Keenan, F.P.: 2004, Propagating waves and magnetohydrodynamic mode coupling in the quiet-Sun network. Astrophys. J. 604, 936. DOI. ADS.

Boe, B., Habbal, S., Druckmüller, M., Landi, E., Kourkchi, E., Ding, A., Starha, P., Hutton, J.: 2018, The first empirical determination of the $\mathrm{Fe}^{10+}$ and $\mathrm{Fe}^{13+}$ freeze-in distances in the solar corona. Astrophys. J. 859, 155. DOI. ADS.

Bogdan, T.J.: 2000, Sunspot oscillations: a review - (invited review). Solar Phys. 192, 373. DOI. ADS.

Bonet, J.A., Márquez, I., Sánchez Almeida, J., Cabello, I., Domingo, V.: 2008, Convectively driven vortex flows in the Sun. Astrophys. J. Lett. 687, L131. DOI. ADS.

Borrero, J.M., Ichimoto, K.: 2011, Magnetic structure of sunspots. Liv. Rev. Solar Phys. 8, 4. DOI. ADS.

Borrero, J.M., Jafarzadeh, S., Schüssler, M., Solanki, S.K.: 2017, Solar magnetoconvection and small-scale dynamo. Recent developments in observation and simulation. Space Sci. Rev. 210, 275. DOI. ADS.

Bose, S., Henriques, V.M.J., Rouppe van der Voort, L., Pereira, T.M.D.: 2019, Semi-empirical model atmospheres for the chromosphere of the sunspot penumbra and umbral flashes. Astron. Astrophys. 627, A46. DOI. ADS.

Braun, D.C.: 1995, Scattering of p-modes by sunspots. I. Observations. Astrophys. J. 451, 859. DOI. ADS.

Braun, D.C., Duvall, T.L. Jr., Labonte, B.J.: 1987, Acoustic absorption by sunspots. Astrophys. J. Lett. 319, L27. DOI. ADS.

Braun, D.C., Duvall, T.L. Jr., Labonte, B.J.: 1988, The absorption of high-degree p-mode oscillations in and around sunspots. Astrophys. J. 335, 1015. DOI. ADS.

Braun, D.C., Duvall, T.L. Jr., Labonte, B.J., Jefferies, S.M., Harvey, J.W., Pomerantz, M.A.: 1992, Scattering of p-modes by a sunspot. Astrophys. J. Lett. 391, L113. DOI. ADS.

Brooks, D.H., Warren, H.P.: 2011, Establishing a connection between active region outflows and the solar wind: abundance measurements with EIS/Hinode. Astrophys. J. Lett. 727, L13. DOI. ADS.

Brooks, D.H., Ugarte-Urra, I., Warren, H.P.: 2015, Full-Sun observations for identifying the source of the slow solar wind. Nat. Comm. 6, 5947. DOI. ADS.

Brouillette, M.: 2002, The Richtmyer-Meshkov instability. Annu. Rev. Fluid Mech. 34, 445. DOI. ADS.

Brown, D.S., Nightingale, R.W., Alexander, D., Schrijver, C.J., Metcalf, T.R., Shine, R.A., Title, A.M., Wolfson, C.J.: 2003, Observations of rotating sunspots from TRACE. Solar Phys. 216, 79. DOI. ADS.

Brown, J.C., Carlson, R.W., Toner, M.P.: 2015, Destruction and observational signatures of Sun-impacting comets. Astrophys. J. 807, 165. DOI. ADS.

Brun, A.S., Browning, M.K.: 2017, Magnetism, dynamo action and the solar-stellar connection. Liv. Rev. Solar Phys. 14, 4. DOI. ADS.

Bryans, P., Pesnell, W.D.: 2012, The extreme-ultraviolet emission from Sun-grazing comets. Astrophys. J. 760, 18. DOI. ADS.

Bryans, P., Pesnell, W.D.: 2016, On the absence of EUV emission from comet C/2012 S1 (ISON). Astrophys. J. 822, 77. DOI. ADS. 
Buccino, A.P., Lemarchand, G.A., Mauas, P.J.D.: 2007, UV habitable zones around M stars. Icarus 192, 582. DOI. ADS.

Burch, J.L., Torbert, R.B., Phan, T.D., Chen, L.-J., Moore, T.E., Ergun, R.E., Eastwood, J.P., Gershman, D.J., Cassak, P.A., Argall, M.R., Wang, S., Hesse, M., Pollock, C.J., Giles, B.L., Nakamura, R., Mauk, B.H., Fuselier, S.A., Russell, C.T., Strangeway, R.J., Drake, J.F., Shay, M.A., Khotyaintsev, Y.V., Lindqvist, P.-A., Marklund, G., Wilder, F.D., Young, D.T., Torkar, K., Goldstein, J., Dorelli, J.C., Avanov, L.A., Oka, M., Baker, D.N., Jaynes, A.N., Goodrich, K.A., Cohen, I.J., Turner, D.L., Fennell, J.F., Blake, J.B., Clemmons, J., Goldman, M., Newman, D., Petrinec, S.M., Trattner, K.J., Lavraud, B., Reiff, P.H., Baumjohann, W., Magnes, W., Steller, M., Lewis, W., Saito, Y., Coffey, V., Chandler, M.: 2016, Electronscale measurements of magnetic reconnection in space. Science 352, aaf2939. DOI. ADS.

Burger, M.H., Killen, R.M., McClintock, W.E., Vervack, R.J. Jr., Merkel, A.W., Sprague, A.L., Sarantos, M.: 2012, Modeling MESSENGER observations of calcium in Mercury's exosphere. J. Geophys. Res., Planets 117, E00L11. DOI. ADS.

Bushby, P.J., Houghton, S.M., Proctor, M.R.E., Weiss, N.O.: 2008, Convective intensification of magnetic fields in the quiet Sun. Mon. Not. Roy. Astron. Soc. 387, 698. DOI. ADS.

Bzowski, M., Królikowska, M.: 2005, Are the sungrazing comets the inner source of pickup ions and energetic neutral atoms? Astron. Astrophys. 435, 723. DOI. ADS.

Cally, P.S., Bogdan, T.J.: 1997, Simulation of f- and p-mode interactions with a stratified magnetic field concentration. Astrophys. J. Lett. 486, L67. DOI. ADS.

Cally, P.S., Bogdan, T.J., Zweibel, E.G.: 1994, Umbral oscillations in sunspots: absorption of p-modes and active region heating by mode conversion. Astrophys. J. 437, 505. DOI. ADS.

Cameron, R.H., Dikpati, M., Brandenburg, A.: 2017, The global solar dynamo. Space Sci. Rev. $210,367$. DOI. ADS.

Canfield, R.C., Hudson, H.S., McKenzie, D.E.: 1999, Sigmoidal morphology and eruptive solar activity. Geophys. Res. Lett. 26, 627. DOI. ADS.

Carlsson, M., Stein, R.F.: 1997, Formation of solar calcium H and K bright grains. Astrophys. J. 481, 500. DOI. ADS.

Carlsson, M., Stein, R.F.: 2002, Wave processes in the solar upper atmosphere. In: Sawaya-Lacoste, H. (ed.) SOLMAG 2002. Proceedings of the Magnetic Coupling of the Solar Atmosphere Euroconference CS505, ESA, Noordwijk, 293. ADS.

Carlsson, M., De Pontieu, B., Hansteen, V.H.: 2019, New view of the solar chromosphere. Annu. Rev. Astron. Astrophys. 57, 189. DOI. ADS.

Caselli, P., Walmsley, C.M., Terzieva, R., Herbst, E.: 1998, The ionization fraction in dense cloud cores. Astrophys. J. 499, 234. DOI. ADS.

Casini, R., López Ariste, A., Tomczyk, S., Lites, B.W.: 2003, Magnetic maps of prominences from full Stokes analysis of the He I D3 line. Astrophys. J. Lett. 598, L67. DOI. ADS.

Cassidy, T.A., Merkel, A.W., Burger, M.H., Sarantos, M., Killen, R.M., McClintock, W.E., Vervack, R.J.: 2015, Mercury's seasonal sodium exosphere: MESSENGER orbital observations. Icarus 248, 547. DOI. ADS.

Cattaneo, F.: 1999, On the origin of magnetic fields in the quiet photosphere. Astrophys. J. Lett. 515, L39. DOI. ADS.

Cauzzi, G., Reardon, K.P., Uitenbroek, H., Cavallini, F., Falchi, A., Falciani, R., Janssen, K., Rimmele, T., Vecchio, A., Wöger, F.: 2008, The solar chromosphere at high resolution with IBIS. I. New insights from the Ca II $854.2 \mathrm{~nm}$ line. Astron. Astrophys. 480, 515. DOI. ADS.

Cauzzi, G., Reardon, K., Rutten, R.J., Tritschler, A., Uitenbroek, H.: 2009, The solar chromosphere at high resolution with IBIS. IV. Dual-line evidence of heating in chromospheric network. Astron. Astrophys. 503, 577. DOI. ADS.

Centeno, R., Collados, M., Trujillo Bueno, J.: 2006, Oscillations and wave propagation in different solar magnetic features. In: Casini, R., Lites, B.W. (eds.) Solar Polarization 4, Astron. Soc. Pacific, San Francisco CS-358, 465. ADS.

Centeno, R., Collados, M., Trujillo Bueno, J.: 2009, Wave propagation and shock formation in different magnetic structures. Astrophys. J. 692, 1211. DOI. ADS.

Centeno, R., Blanco Rodríguez, J., Del Toro Iniesta, J.C., Solanki, S.K., Barthol, P., Gand orfer, A., Gizon, L., Hirzberger, J., Riethmüller, T.L., van Noort, M., Orozco Suárez, D., Berkefeld, T., Schmidt, W., Martínez Pillet, V., Knölker, M.: 2017, A tale of two emergences: sunrise II observations of emergence sites in a solar active region. Astrophys. J. Suppl. Ser. 229, 3. DOI. ADS.

Chae, J.: 2001, Observational determination of the rate of magnetic helicity transport through the solar surface via the horizontal motion of field line footpoints. Astrophys. J. Lett. 560, L95. DOI. ADS.

Chandorkar, M., Furtlehner, C., Poduval, B., Camporeale, E., Sebag, M.: 2020, Dynamic time lag regression: predicting what \& when. In: International Conference on Learning Representations. ir.cwi.nl/pub/ 30464. 
Chaplin, W.J., Elsworth, Y., Isaak, G.R., Lines, R., McLeod, C.P., Miller, B.A., New, R.: 1998, Solar p-mode excitation: further insight from recent low-1 BiSON helioseismological data. Mon. Not. Roy. Astron. Soc. 298, L7. DOI. ADS.

Chapman, G.A., Cookson, A.M., Dobias, J.J.: 1996, Variations in total solar irradiance during solar cycle 22. J. Geophys. Res. 101, 13541. DOI. ADS.

Charbonneau, P.: 2010, Dynamo models of the solar cycle. Liv. Rev. Solar Phys. 7, 3. DOI. ADS.

Charbonneau, P.: 2014, Solar dynamo theory. Annu. Rev. Astron. Astrophys. 52, 251. DOI. ADS.

Charbonneau, P.: 2016, Solar physics: dynamo theory questioned. Nature 535, 500. DOI. ADS.

Chen, P.F.: 2011, Coronal mass ejections: models and their observational basis. Liv. Rev. Solar Phys. 8, 1. DOI. ADS.

Chen, Z.Z., Wang, T. Y., Yu, Y., Chen, F.: 2020, Relationship between current filaments and turbulence during a turbulent reconnection. Astrophys. J. Lett. 888, L16. DOI. ADS.

Chitta, L.P., Peter, H., Young, P.R.: 2016, A closer look at a coronal loop rooted in a sunspot umbra. Astron. Astrophys. 587, A20. DOI. ADS.

Chiueh, T.: 1998, Ambipolar diffusion-driven tearing instability in a steepening background magnetic field. Astrophys. J. 494, 90. DOI. ADS.

Cirtain, J.W., Golub, L., Winebarger, A.R., De Pontieu, B., Kobayashi, K., Moore, R.L., Walsh, R.W., Korreck, K.E., Weber, M., McCauley, P., Title, A., Kuzin, S., Deforest, C.E.: 2013, Energy release in the solar corona from spatially resolved magnetic braids. Nature 493, 501. DOI. ADS.

Coates, A.J.: 2016, Connecting the dots in magnetic reconnection. Science 352, 1176. DOI. ADS.

Cossette, J.-F., Rast, M.P.: 2016, Supergranulation as the largest buoyantly driven convective scale of the Sun. Astrophys. J. Lett. 829, L17. DOI. ADS.

Cranmer, S.R.: 2009, Coronal holes. Liv. Rev. Solar Phys. 6, 3. DOI. ADS.

Cranmer, S.R., van Ballegooijen, A.A.: 2005, On the generation, propagation, and reflection of Alfvén waves from the solar photosphere to the distant heliosphere. Astrophys. J. Suppl. Ser. 156, 265. DOI. ADS.

Cranmer, S.R., Winebarger, A.R.: 2019, The properties of the solar corona and its connection to the solar wind. Annu. Rev. Astron. Astrophys. 57, 157. DOI. ADS.

Cremonese, G., Huebner, W.F., Rauer, H., Boice, D.C.: 2002, Neutral sodium tails in comets. Adv. Space Res. 29, 1187. DOI. ADS.

Criscuoli, S.: 2013, Comparison of physical properties of quiet and active regions through the analysis of magnetohydrodynamic simulations of the solar photosphere. Astrophys. J. 778, 27. DOI. ADS.

Criscuoli, S., Foukal, P.: 2017, A study of solar photospheric temperature gradient variation using limb darkening measurements. Astrophys. J. 835, 99. DOI. ADS.

Criscuoli, S., Rast, M.P.: 2009, Photometric properties of resolved and unresolved magnetic elements. Astron. Astrophys. 495, 621. DOI. ADS.

Criscuoli, S., Norton, A., Whitney, T.: 2017, Photometric properties of network and faculae derived from HMI data compensated for scattered light. Astrophys. J. 847, 93. DOI. ADS.

Danilovic, S.: 2017, Simulating Ellerman bomb-like events. Astron. Astrophys. 601, A122. DOI. ADS.

Danilovic, S., Schüssler, M., Solanki, S.K.: 2010, Probing quiet Sun magnetism using MURaM simulations and Hinode/SP results: support for a local dynamo. Astron. Astrophys. 513, A1. DOI. ADS.

Davey, A., et al.: 2021, The DKIST data center. Solar Phys. 296 (in preparation).

de la Cruz Rodríguez, J., Socas-Navarro, H.: 2011, Are solar chromospheric fibrils tracing the magnetic field? Astron. Astrophys. 527, L8. DOI. ADS.

de la Cruz Rodríguez, J., Leenaarts, J., Asensio Ramos, A.: 2016, Non-LTE inversions of the Mg II h \& k and UV triplet lines. Astrophys. J. Lett. 830, L30. DOI. ADS.

de la Cruz Rodríguez, J., Rouppe van der Voort, L., Socas-Navarro, H., van Noort, M.: 2013, Physical properties of a sunspot chromosphere with umbral flashes. Astron. Astrophys. 556, A115. DOI. ADS.

De Moortel, I.: 2009, Longitudinal waves in coronal loops. Space Sci. Rev. 149, 65. DOI. ADS.

De Moortel, I., Browning, P.: 2015, Recent advances in coronal heating. Phil. Trans. Roy. Soc. London Ser. A 373, 20140269. DOI. ADS.

De Pontieu, B., Erdélyi, R., James, S.P.: 2004, Solar chromospheric spicules from the leakage of photospheric oscillations and flows. Nature 430, 536. DOI. ADS.

De Pontieu, B., Martens, P.C.H., Hudson, H.S.: 2001, Chromospheric damping of Alfvén waves. Astrophys. J. 558, 859. DOI. ADS.

De Pontieu, B., McIntosh, S., Hansteen, V.H., Carlsson, M., Schrijver, C.J., Tarbell, T.D., Title, A.M., Shine, R.A., Suematsu, Y., Tsuneta, S., Katsukawa, Y., Ichimoto, K., Shimizu, T., Nagata, S.: 2007a, A tale of two spicules: the impact of spicules on the magnetic chromosphere. Publ. Astron. Soc. Japan 59, S655. DOI. ADS.

De Pontieu, B., McIntosh, S.W., Carlsson, M., Hansteen, V.H., Tarbell, T.D., Schrijver, C.J., Title, A.M., Shine, R.A., Tsuneta, S., Katsukawa, Y., Ichimoto, K., Suematsu, Y., Shimizu, T., Nagata, S.: 2007b, Chromospheric Alfvénic waves strong enough to power the solar wind. Science 318, 1574. DOI. ADS. 
De Pontieu, B., McIntosh, S.W., Hansteen, V.H., Schrijver, C.J.: 2009, Observing the roots of solar coronal heating - in the chromosphere. Astrophys. J. Lett. 701, L1. DOI. ADS.

De Pontieu, B., McIntosh, S.W., Carlsson, M., Hansteen, V.H., Tarbell, T.D., Boerner, P., Martinez-Sykora, J., Schrijver, C.J., Title, A.M.: 2011, The origins of hot plasma in the solar corona. Science 331, 55. DOI. ADS.

De Pontieu, B., Rouppe van der Voort, L., McIntosh, S.W., Pereira, T.M.D., Carlsson, M., Hansteen, V., Skogsrud, H., Lemen, J., Title, A., Boerner, P., Hurlburt, N., Tarbell, T.D., Wuelser, J.P., De Luca, E.E., Golub, L., McKillop, S., Reeves, K., Saar, S., Testa, P., Tian, H., Kankelborg, C., Jaeggli, S., Kleint, L., Martinez-Sykora, J.: 2014a, On the prevalence of small-scale twist in the solar chromosphere and transition region. Science 346, 1255732. DOI. ADS.

De Pontieu, B., Title, A.M., Lemen, J.R., Kushner, G.D., Akin, D.J., Allard, B., Berger, T., Boerner, P., Cheung, M., Chou, C., Drake, J.F., Duncan, D.W., Freeland, S., Heyman, G.F., Hoffman, C., Hurlburt, N.E., Lindgren, R.W., Mathur, D., Rehse, R., Sabolish, D., Seguin, R., Schrijver, C.J., Tarbell, T.D., Wülser, J.-P., Wolfson, C.J., Yanari, C., Mudge, J., Nguyen-Phuc, N., Timmons, R., van Bezooijen, R., Weingrod, I., Brookner, R., Butcher, G., Dougherty, B., Eder, J., Knagenhjelm, V., Larsen, S., Mansir, D., Phan, L., Boyle, P., Cheimets, P.N., DeLuca, E.E., Golub, L., Gates, R., Hertz, E., McKillop, S., Park, S., Perry, T., Podgorski, W.A., Reeves, K., Saar, S., Testa, P., Tian, H., Weber, M., Dunn, C., Eccles, S., Jaeggli, S.A., Kankelborg, C.C., Mashburn, K., Pust, N., Springer, L., Carvalho, R., Kleint, L., Marmie, J., Mazmanian, E., Pereira, T.M.D., Sawyer, S., Strong, J., Worden, S.P., Carlsson, M., Hansteen, V.H., Leenaarts, J., Wiesmann, M., Aloise, J., Chu, K.-C., Bush, R.I., Scherrer, P.H., Brekke, P., Martinez-Sykora, J., Lites, B.W., McIntosh, S.W., Uitenbroek, H., Okamoto, T.J., Gummin, M.A., Auker, G., Jerram, P., Pool, P., Waltham, N.: 2014b, The Interface Region Imaging Spectrograph (IRIS). Solar Phys. 289, 2733. DOI. ADS.

De Pontieu, B., McIntosh, S., Martinez-Sykora, J., Peter, H., Pereira, T.M.D.: 2015, Why is non-thermal line broadening of spectral lines in the lower transition region of the Sun independent of spatial resolution? Astrophys. J. Lett. 799, L12. DOI. ADS.

De Pontieu, B., De Moortel, I., Martinez-Sykora, J., McIntosh, S.W.: 2017, Observations and numerical models of solar coronal heating associated with spicules. Astrophys. J. Lett. 845, L18. DOI. ADS.

de Wijn, A.G., et al.: 2021, The DKIST Visible Spectro-Polarimeter (ViSP). Solar Phys. 296 (in preparation).

de Wijn, A.G., Stenflo, J.O., Solanki, S.K., Tsuneta, S.: 2009, Small-scale solar magnetic fields. Space Sci. Rev. 144, 275. DOI. ADS.

Del Zanna, G., DeLuca, E.E.: 2018, Solar coronal lines in the visible and infrared: a rough guide. Astrophys. J. 852, 52. DOI. ADS.

Delmont, P., Keppens, R.: 2011, Parameter regimes for slow, intermediate and fast MHD shocks. J. Plasma Phys. 77, 207. DOI. ADS.

Démoulin, P.: 2007, Recent theoretical and observational developments in magnetic helicity studies. Adv. Space Res. 39, 1674. DOI. ADS.

Démoulin, P., Pariat, E.: 2009, Modelling and observations of photospheric magnetic helicity. Adv. Space Res. 43, 1013. DOI. ADS.

DeVore, C.R.: 2000, Magnetic helicity generation by solar differential rotation. Astrophys. J. 539, 944. DOI. ADS.

Díaz Baso, C.J., Martínez González, M.J., Asensio Ramos, A.: 2019a, Spectropolarimetric analysis of an active region filament. I. Magnetic and dynamical properties from single component inversions. Astron. Astrophys. 625, A128. DOI. ADS.

Díaz Baso, C.J., Martínez González, M.J., Asensio Ramos, A.: 2019b, Spectropolarimetric analysis of an active region filament. II. Evidence of the limitations of a single-component model. Astron. Astrophys. 625, A129. DOI. ADS.

Dima, G., Kuhn, J., Berdyugina, S.: 2016, Infrared dual-line Hanle diagnostic of the coronal vector magnetic field. Front. Astron. Space Sci. 3, 13. DOI. ADS.

Domingue, D.L., Koehn, P.L., Killen, R.M., Sprague, A.L., Sarantos, M., Cheng, A.F., Bradley, E.T., McClintock, W.E.: 2007, Mercury's atmosphere: a surface-bounded exosphere. Space Sci. Rev. 131, 161. DOI. ADS.

Donea, A.-C., Lindsey, C.: 2005, Seismic emission from the solar flares of 2003 October 28 and 29. Astrophys. J. 630, 1168. DOI. ADS.

Dong, Q.-L., Wang, S.-J., Lu, Q.-M., Huang, C., Yuan, D.-W., Liu, X., Lin, X.-X., Li, Y.-T., Wei, H.-G., Zhong, J.-Y., Shi, J.-R., Jiang, S.-E., Ding, Y.-K., Jiang, B.-B., Du, K., He, X.-T., Yu, M.Y., Liu, C.S., Wang, S., Tang, Y.-J., Zhu, J.-Q., Zhao, G., Sheng, Z.-M., Zhang, J.: 2012, Plasmoid ejection and secondary current sheet generation from magnetic reconnection in laser-plasma interaction. Phys. Rev. Lett. 108, 215001. DOI. ADS.

Dove, J.B., Gibson, S.E., Rachmeler, L.A., Tomczyk, S., Judge, P.: 2011, A ring of polarized light: evidence for twisted coronal magnetism in cavities. Astrophys. J. Lett. 731, L1. DOI. ADS. 
Downs, C., Linker, J.A., Mikić, Z., Riley, P., Schrijver, C.J., Saint-Hilaire, P.: 2013, Probing the solar magnetic field with a Sun-grazing comet. Science 340, 1196. DOI. ADS.

Doyle, L., Wyper, P.F., Scullion, E., McLaughlin, J.A., Ramsay, G., Doyle, J.G.: 2019, Observations and 3D magnetohydrodynamic modeling of a confined helical jet launched by a filament eruption. Astrophys. J. $\mathbf{8 8 7}, 246$. DOI. ADS.

Druckmüller, M., Habbal, S.R., Aniol, P., Ding, A., Morgan, H.: 2014, Imaging comet ISON C/2012 S1 in the inner corona at perihelion. Astrophys. J. Lett. 784, L22. DOI. ADS.

Druett, M., Scullion, E., Zharkova, V., Matthews, S., Zharkov, S., Rouppe van der Voort, L.: 2017, Beam electrons as a source of $\mathrm{H} \alpha$ flare ribbons. Nat. Commun. 8, 15905. DOI. ADS.

Dudík, J., Janvier, M., Aulanier, G., Del Zanna, G., Karlický, M., Mason, H.E., Schmieder, B.: 2014, Slipping magnetic reconnection during an X-class solar flare observed by SDO/AIA. Astrophys. J. 784, 144. DOI. ADS.

Dudok de Wit, T., Krasnoselskikh, V.V., Bale, S.D., Bonnell, J.W., Bowen, T.A., Chen, C.H.K., Froment, C., Goetz, K., Harvey, P.R., Jagarlamudi, V.K., Larosa, A., MacDowall, R.J., Malaspina, D.M., Matthaeus, W.H., Pulupa, M., Velli, M., Whittlesey, P.L.: 2020, Switchbacks in the near-Sun magnetic field: long memory and impact on the turbulence cascade. Astrophys. J. Suppl. Ser. 246, 39. DOI. ADS.

Duvall, T.L. Jr., Jefferies, S.M., Harvey, J.W., Osaki, Y., Pomerantz, M.A.: 1993, Asymmetries of solar oscillation line profiles. Astrophys. J. 410, 829. DOI. ADS.

Ebert, R.W., McComas, D.J., Elliott, H.A., Forsyth, R.J., Gosling, J.T.: 2009, Bulk properties of the slow and fast solar wind and interplanetary coronal mass ejections measured by Ulysses: three polar orbits of observations. J. Geophys. Res. Space Phys. 114, A01109. DOI. ADS.

Edwin, P.M., Roberts, B.: 1982, Wave propagation in a magnetically structured atmosphere - part three - the slab in a magnetic environment. Solar Phys. 76, 239. DOI. ADS.

Edwin, P.M., Roberts, B.: 1983, Wave propagation in a magnetic cylinder. Solar Phys. 88, 179. DOI. ADS.

Ellerman, F.: 1917, Solar hydrogen "bombs". Astrophys. J. 46, 298. DOI. ADS.

Erdélyi, R., Ballai, I.: 2007, Heating of the solar and stellar coronae: a review. Astron. Nachr. 328, 726. DOI. ADS.

Erdélyi, R., Fedun, V.: 2007, Are there Alfvén waves in the solar atmosphere? Science 318, 1572. DOI. ADS.

Ermolli, I., Matthes, K., Dudok de Wit, T., Krivova, N.A., Tourpali, K., Weber, M., Unruh, Y.C., Gray, L., Langematz, U., Pilewskie, P., Rozanov, E., Schmutz, W., Shapiro, A., Solanki, S.K., Woods, T.N.: 2013, Recent variability of the solar spectral irradiance and its impact on climate modelling. Atmos. Chem. Phys. 13, 3945. DOI. ADS.

Esteban Pozuelo, S., Bellot Rubio, L.R., de la Cruz Rodríguez, J.: 2015, Lateral downflows in sunspot penumbral filaments and their temporal evolution. Astrophys. J. 803, 93. DOI. ADS.

Esteban Pozuelo, S.E., Bellot Rubio, L.R., de la Cruz Rodríguez, J.: 2016, Properties of supersonic evershed downflows. Astrophys. J. 832, 170. DOI. ADS.

Evershed, J.: 1909, Radial movement in sun-spots. Mon. Not. Roy. Astron. Soc. 69, 454. DOI. ADS.

Fan, Y., Gibson, S.E.: 2007, Onset of coronal mass ejections due to loss of confinement of coronal flux ropes. Astrophys. J. 668, 1232. DOI. ADS.

Fang, X., Xia, C., Keppens, R.: 2013, Multidimensional modeling of coronal rain dynamics. Astrophys. J. Lett. 771, L29. DOI. ADS.

Faurobert, M., Balasubramanian, R., Ricort, G.: 2016, Variation of the temperature gradient in the solar photosphere with magnetic activity. Astron. Astrophys. 595, A71. DOI. ADS.

Faurobert, M., Carbillet, M., Marquis, L., Chiavassa, A., Ricort, G.: 2018, Temperature gradient in the solar photosphere. Test of a new spectroscopic method and study of its feasibility for ground-based telescopes. Astron. Astrophys. 616, A133. DOI. ADS.

Fehlmann, A., et al.: 2021, The DKIST Cryogenic Near-Infrared Spectropolarimeter (Cryo-NIRSP). Solar Phys. 296 (in preparation).

Feldman, U.: 1992, Elemental abundances in the upper solar atmosphere. Phys. Scr. 46, 202. DOI. ADS.

Feldman, U., Laming, J.M.: 2000, Element abundances in the upper atmospheres of the Sun and stars: update of observational results. Phys. Scr. 61, 222. DOI. ADS.

Feldman, U., Landi, E., Schwadron, N.A.: 2005, On the sources of fast and slow solar wind. J. Geophys. Res. Space Phys. 110, A07109. DOI. ADS.

Felipe, T., Khomenko, E., Collados, M.: 2011, Magnetoacoustic wave energy from numerical simulations of an observed sunspot umbra. Astrophys. J. 735, 65. DOI. ADS.

Finsterle, W., Jefferies, S.M., Cacciani, A., Rapex, P., McIntosh, S.W.: 2004a, Helioseismic mapping of the magnetic canopy in the solar chromosphere. Astrophys. J. Lett. 613, L185. DOI. ADS.

Finsterle, W., Jefferies, S.M., Cacciani, A., Rapex, P., Giebink, C., Knox, A., Dimartino, V.: 2004b, Seismology of the solar atmosphere. Solar Phys. 220, 317. DOI. ADS.

Fisher, G.H., Welsch, B.T.: 2008, FLCT: a fast, efficient method for performing local correlation tracking. In: Howe, R., Komm, R.W., Balasubramaniam, K.S., Petrie, G.J.D. (eds.) Subsurface and Atmospheric Influences on Solar Activity CS-383, Astron. Soc. Pacific, San Francisco, 373. ADS. 
Fisher, G.H., Welsch, B.T., Abbett, W.P.: 2012, Can we determine electric fields and Poynting fluxes from vector magnetograms and Doppler measurements? Solar Phys. 277, 153. DOI. ADS.

Fisk, L.A., Kasper, J.C.: 2020, Global circulation of the open magnetic flux of the Sun. Astrophys. J. Lett. 894, L4. DOI. ADS.

Fleishman, G., Mysh’yakov, I., Stupishin, A., Loukitcheva, M., Anfinogentov, S.: 2019, Force-free field reconstructions enhanced by chromospheric magnetic field data. Astrophys. J. 870, 101. DOI. ADS.

Fletcher, L.: 2009, Ultra-violet footpoints as tracers of coronal magnetic connectivity and restructuring during a solar flare. Astron. Astrophys. 493, 241. DOI. ADS.

Fletcher, L., Hannah, I.G., Hudson, H.S., Metcalf, T.R.: 2007, A TRACE white light and RHESSI hard X-ray study of flare energetics. Astrophys. J. 656, 1187. DOI. ADS.

Fletcher, L., Hannah, I.G., Hudson, H.S., Innes, D.E.: 2013, Flare ribbon energetics in the early phase of an SDO flare. Astrophys. J. 771, 104. DOI. ADS.

Forbes, T.G., Priest, E.R.: 1983a, A numerical experiment relevant to line-tied reconnection in two-ribbon flares. Solar Phys. 84, 169. DOI. ADS.

Forbes, T.G., Priest, E.R.: 1983b, On reconnection and plasmoids in the geomagnetic tail. J. Geophys. Res. 88, 863. DOI. ADS.

Fossat, E., Gelly, B., Grec, G., Pomerantz, M.: 1987, Search for solar P-mode frequency changes between 1980 and 1985. Astron. Astrophys. 177, L47. ADS.

France, K., Froning, C.S., Linsky, J.L., Roberge, A., Stocke, J.T., Tian, F., Bushinsky, R., Désert, J.-M., Mauas, P., Vieytes, M., Walkowicz, L.M.: 2013, The ultraviolet radiation environment around M dwarf exoplanet host stars. Astrophys. J. 763, 149. DOI. ADS.

Freij, N., Scullion, E.M., Nelson, C.J., Mumford, S., Wedemeyer, S., Erdélyi, R.: 2014, The detection of upwardly propagating waves channeling energy from the chromosphere to the low corona. Astrophys. J. 791, 61. DOI. ADS.

French, R.J., Judge, P.G., Matthews, S.A., van Driel-Gesztelyi, L.: 2019, Spectropolarimetric insight into plasma sheet dynamics of a solar flare. Astrophys. J. Lett. 887, L34. DOI. ADS.

Frisch, U., Pouquet, A., Leorat, J., Mazure, A.: 1975, Possibility of an inverse cascade of magnetic helicity in magnetohydrodynamic turbulence. J. Fluid Mech. 68, 769. DOI. ADS.

Froment, C., Auchère, F., Mikić, Z., Aulanier, G., Bocchialini, K., Buchlin, E., Solomon, J., Soubrié, E.: 2018, On the occurrence of thermal nonequilibrium in coronal loops. Astrophys. J. 855, 52. DOI. ADS.

Gabriel, A.H.: 1976, A magnetic model of the solar transition region. Phil. Trans. Roy. Soc. London Ser. A 281, 339. DOI. ADS.

Gabriel, M.: 1992, On the solar p-mode spectrum excited by convection. Astron. Astrophys. 265, 771. ADS.

Gabriel, M.: 1995, On the profile of the solar p-mode lines. Astron. Astrophys. 299, 245. ADS.

Geiss, J., Gloeckler, G., von Steiger, R.: 1995, Origin of the solar wind from composition data. Space Sci. Rev. 72, 49. DOI. ADS.

Gekelman, W., Pribyl, P., Lucky, Z., Drandell, M., Leneman, D., Maggs, J., Vincena, S., Van Compernolle, B., Tripathi, S.K.P., Morales, G., Carter, T.A., Wang, Y., DeHaas, T.: 2016, The upgraded Large Plasma Device, a machine for studying frontier basic plasma physics. Rev. Sci. Instrum. 87, 025105. DOI. ADS.

Georgoulis, M.K., Rust, D.M., Bernasconi, P.N., Schmieder, B.: 2002, Statistics, morphology, and energetics of Ellerman bombs. Astrophys. J. 575, 506. DOI. ADS.

Giagkiozis, I., Fedun, V., Scullion, E., Jess, D.B., Verth, G.: 2018, Vortex flows in the solar atmosphere: automated identification and statistical analysis. Astrophys. J. 869, 169. DOI. ADS.

Gibson, S.E.: 2018, Solar prominences: theory and models. Fleshing out the magnetic skeleton. Liv. Rev. Solar Phys. 15, 7. DOI. ADS.

Gizon, L., Birch, A.C.: 2002, Time-distance helioseismology: the forward problem for random distributed sources. Astrophys. J. 571, 966. DOI. ADS.

Gizon, L., Birch, A.C., Spruit, H.C.: 2010, Local helioseismology: three-dimensional imaging of the solar interior. Annu. Rev. Astron. Astrophys. 48, 289. DOI. ADS.

Goldreich, P., Keeley, D.A.: 1977, Solar seismology. II. The stochastic excitation of the solar p-modes by turbulent convection. Astrophys. J. 212, 243. DOI. ADS.

Goldreich, P., Kumar, P.: 1990, Wave generation by turbulent convection. Astrophys. J. 363, 694. DOI. ADS.

Goldreich, P., Murray, N., Kumar, P.: 1994, Excitation of solar p-modes. Astrophys. J. 424, 466. DOI. ADS.

Goode, P.R., Strous, L.H., Rimmele, T.R., Stebbins, R.T.: 1998, On the origin of solar oscillations. Astrophys. J. Lett. 495, L27. DOI. ADS.

Goodman, M.L.: 2012, Acceleration of type II spicules in the solar chromosphere. Astrophys. J. 757, 188. DOI. ADS.

Goossens, M., Erdélyi, R., Ruderman, M.S.: 2011, Resonant MHD waves in the solar atmosphere. Space Sci. Rev. 158, 289. DOI. ADS.

Gošić, M., Bellot Rubio, L.R., del Toro Iniesta, J.C., Orozco Suárez, D., Katsukawa, Y.: 2016, The solar internetwork. II. Flux appearance and disappearance rates. Astrophys. J. 820, 35. DOI. ADS. 
Graham, D.R., Cauzzi, G.: 2015, Temporal evolution of multiple evaporating ribbon sources in a solar flare. Astrophys. J. Lett. 807, L22. DOI. ADS.

Graham, D.R., Fletcher, L., Labrosse, N.: 2015, Determining energy balance in the flaring chromosphere from oxygen V line ratios. Astron. Astrophys. 584, A6. DOI. ADS.

Gray, D.F.: 2005, The Observation and Analysis of Stellar Photospheres, Cambridge Univ. Press, Cambridge. ADS.

Green, L.M., Török, T., Vršnak, B., Manchester, W., Veronig, A.: 2018, The origin, early evolution and predictability of solar eruptions. Space Sci. Rev. 214, 46. DOI. ADS.

Grossmann-Doerth, U., Schüssler, M., Steiner, O.: 1998, Convective intensification of solar surface magnetic fields: results of numerical experiments. Astron. Astrophys. 337, 928. ADS.

Guo, L.-J., Bhattacharjee, A., Huang, Y.-M.: 2013, Distribution of plasmoids in post-coronal mass ejection current sheets. Astrophys. J. Lett. 771, L14. DOI. ADS.

Gupta, G.R.: 2017, Spectroscopic evidence of Alfvén wave damping in the off-limb solar corona. Astrophys. J. 836, 4. DOI. ADS.

Haber, D.A., Hindman, B.W., Toomre, J., Bogart, R.S., Larsen, R.M., Hill, F.: 2002, Evolving submerged meridional circulation cells within the upper convection zone revealed by ring-diagram analysis. Astrophys. J. 570, 855. DOI. ADS.

Hagenaar, H.J., Shine, R.A.: 2005, Moving magnetic features around sunspots. Astrophys. J. 635, 659. DOI. ADS.

Hagenaar, H.J., DeRosa, M.L., Schrijver, C.J.: 2008, The dependence of ephemeral region emergence on local flux imbalance. Astrophys. J. 678, 541. DOI. ADS.

Hahn, M., Landi, E., Savin, D.W.: 2012, Evidence of wave damping at low heights in a polar coronal hole. Astrophys. J. 753, 36. DOI. ADS.

Hale, G.E.: 1908a, On the probable existence of a magnetic field in sun-spots. Astrophys. J. 28, 315 . DOI. ADS.

Hale, G.E.: 1908b, Solar vortices (contributions from the Mt. Wilson Solar Observatory, no. 26). Astrophys. J. 28, 100. DOI. ADS.

Hansteen, V.H., Carlsson, M., Gudiksen, B.: 2007, 3D numerical models of the chromosphere, transition region, and corona. In: Heinzel, P., Dorotovič, I., Rutten, R.J. (eds.) The Physics of Chromospheric Plasmas CS-368, Astron. Soc. Pacific, San Francisco, 107. ADS.

Hansteen, V.H., De Pontieu, B., Rouppe van der Voort, L., van Noort, M., Carlsson, M.: 2006, Dynamic fibrils are driven by magnetoacoustic shocks. Astrophys. J. Lett. 647, L73. DOI. ADS.

Hansteen, V.H., Archontis, V., Pereira, T.M.D., Carlsson, M., Rouppe van der Voort, L., Leenaarts, J.: 2017, Bombs and flares at the surface and lower atmosphere of the Sun. Astrophys. J. 839, 22. DOI. ADS.

Hansteen, V., De Pontieu, B., Carlsson, M., Lemen, J., Title, A., Boerner, P., Hurlburt, N., Tarbell, T.D., Wuelser, J.P., Pereira, T.M.D., De Luca, E.E., Golub, L., McKillop, S., Reeves, K., Saar, S., Testa, P., Tian, H., Kankelborg, C., Jaeggli, S., Kleint, L., Martínez-Sykora, J.: 2014, The unresolved fine structure resolved: IRIS observations of the solar transition region. Science 346, 1255757. DOI. ADS.

Harder, J.W., Fontenla, J.M., Pilewskie, P., Richard, E.C., Woods, T.N.: 2009, Trends in solar spectral irradiance variability in the visible and infrared. Geophys. Res. Lett. 36, L07801. DOI. ADS.

Harra, L.K., Williams, D.R., Wallace, A.J., Magara, T., Hara, H., Tsuneta, S., Sterling, A.C., Doschek, G.A.: 2009, Coronal nonthermal velocity following helicity injection before an X-class flare. Astrophys. J. Lett. 691, L99. DOI. ADS.

Harrington, D., et al.: 2021, The DKIST Polarimetric Calibration. Solar Phys. 296 (in preparation).

Harrington, D.M., Sueoka, S.R., White, A.J.: 2019, Polarization modeling and predictions for Daniel K. Inouye Solar Telescope part 5: impacts of enhanced mirror and dichroic coatings on system polarization calibration. J. Astron. Telesc. Instrum. Syst. 5, 038001. DOI. ADS.

Harrison, R.A., Sime, D.G.: 1989, Comments on coronal mass ejection onset studies. Astron. Astrophys. 208, 274. ADS.

Harrison, R.A., Waggett, P.W., Bentley, R.D., Phillips, K.J.H., Bruner, M., Dryer, M., Simnett, G.M.: 1985, The X-ray signature of solar coronal mass ejections. Rutherford Appleton Laboratory Report 84. ADS.

Harvey, J.: 1999, Hale's discovery of sunspot magnetic fields. Astrophys. J. 525C, 60. ADS.

Heggland, L., De Pontieu, B., Hansteen, V.H.: 2009, Observational signatures of simulated reconnection events in the solar chromosphere and transition region. Astrophys. J. 702, 1. DOI. ADS.

Heinemann, T., Nordlund, Å., Scharmer, G.B., Spruit, H.C.: 2007, MHD simulations of penumbra fine structure. Astrophys. J. 669, 1390. DOI. ADS.

Heinzel, P., Kleint, L.: 2014, Hydrogen balmer continuum in solar flares detected by the Interface Region Imaging Spectrograph (IRIS). Astrophys. J. Lett. 794, L23. DOI. ADS.

Heinzel, P., Kleint, L., Kašparová, J., Krucker, S.: 2017, On the nature of off-limb flare continuum sources detected by SDO/HMI. Astrophys. J. 847, 48. DOI. ADS. 
Henriques, V.M.J., Scullion, E., Mathioudakis, M., Kiselman, D., Gallagher, P.T., Keenan, F.P.: 2015, Stable umbral chromospheric structures. Astron. Astrophys. 574, A131. DOI. ADS.

Henriques, V.M.J., Kuridze, D., Mathioudakis, M., Keenan, F.P.: 2016, Quiet-Sun H $\alpha$ transients and corresponding small-scale transition region and coronal heating. Astrophys. J. 820, 124. DOI. ADS.

Henriques, V.M.J., Mathioudakis, M., Socas-Navarro, H., de la Cruz Rodríguez, J.: 2017, A hot downflowing model atmosphere for umbral flashes and the physical properties of their dark fibrils. Astrophys. J. 845, 102. DOI. ADS.

Henriques, V.M.J., Nelson, C.J., Rouppe van der Voort, L.H.M., Mathioudakis, M.: 2020, Umbral chromospheric fine structure and umbral flashes modelled as one: the corrugated umbra. Astron. Astrophys. 642, A215. DOI. ADS.

Hesse, M., Cassak, P.A.: 2020, Magnetic reconnection in the space sciences: past, present, and future. $J$. Geophys. Res. Space Phys. 125, e25935. DOI. ADS.

Hewitt, R.L., Shelyag, S., Mathioudakis, M., Keenan, F.P.: 2014, Plasma properties and Stokes profiles during the lifetime of a photospheric magnetic bright point. Astron. Astrophys. 565, A84. DOI. ADS.

Heyvaerts, J., Priest, E.R.: 1983, Coronal heating by phase-mixed shear Alfven waves. Astron. Astrophys. 117, 220. ADS.

Hindman, B.W., Zweibel, E.G.: 1994, The effects of a hot outer atmosphere on acoustic-gravity waves. Astrophys. J. 436, 929. DOI. ADS.

Hindman, B.W., Haber, D.A., Toomre, J.: 2006, Helioseismically determined near-surface flows underlying a quiescent filament. Astrophys. J. 653, 725. DOI. ADS.

Hinterreiter, J., Veronig, A.M., Thalmann, J.K., Tschernitz, J., Pötzi, W.: 2018, Statistical properties of ribbon evolution and reconnection electric fields in eruptive and confined flares. Solar Phys. 293, 38. DOI. ADS.

Hollweg, J.V.: 1987, Resonance absorption of magnetohydrodynamic surface waves: physical discussion. Astrophys. J. 312, 880. DOI. ADS.

Hollweg, J.V., Isenberg, P.A.: 2002, Generation of the fast solar wind: a review with emphasis on the resonant cyclotron interaction. J. Geophys. Res. Space Phys. 107, 1147. DOI. ADS.

Hollweg, J.V., Jackson, S., Galloway, D.: 1982, Alfven waves in the solar atmospheres - part three - nonlinear waves on open flux tubes. Solar Phys. 75, 35. DOI. ADS.

Horbury, T.S., Woolley, T., Laker, R., Matteini, L., Eastwood, J., Bale, S.D., Velli, M., Chandran, B.D.G., Phan, T., Raouafi, N.E., Goetz, K., Harvey, P.R., Pulupa, M., Klein, K.G., Dudok de Wit, T., Kasper, J.C., Korreck, K.E., Case, A.W., Stevens, M.L., Whittlesey, P., Larson, D., MacDowall, R.J., Malaspina, D.M., Livi, R.: 2020, Sharp Alfvénic impulses in the near-Sun solar wind. Astrophys. J. Suppl. Ser. 246, 45. DOI. ADS.

Hornig, G., Rastätter, L.: 1997, The role of helicity in the reconnection process. Adv. Space Res. 19, 1789. DOI. ADS.

Howes, G.G.: 2018, Laboratory space physics: investigating the physics of space plasmas in the laboratory. Phys. Plasmas 25, 055501. DOI. ADS.

Hu, F.M., Song, M.T., Li, X.Q.: 1995, $\mathrm{H}_{\alpha}$ filtergram observations of Ellerman bombs and its magnetic reconnection model. Astrophys. Space Sci. 229, 325. DOI. ADS.

Hu, R., Seager, S., Bains, W.: 2012, Photochemistry in terrestrial exoplanet atmospheres. I. Photochemistry model and benchmark cases. Astrophys. J. 761, 166. DOI. ADS.

Hudson, H.S.: 1994, Thermal plasmas in the solar corona: the YOHKOH soft X-ray observations. In: Enome, S., Hirayama, T. (eds.) Proc. of Kofu Symp., Radioheliograph Series, 0911-05501, No. 6; NRO Report No. 36, Nobeyama Radio Observatory, Nobeyama, 1. ADS.

Hudson, H.S., Wolfson, C.J., Metcalf, T.R.: 2006, White-light flares: a TRACE/RHESSI overview. Solar Phys. 234, 79. DOI. ADS.

Hudson, H.S., Fisher, G.H., Welsch, B.T.: 2008, Flare energy and magnetic field variations. In: Howe, R., Komm, R.W., Balasubramaniam, K.S., Petrie, G.J.D., (eds.) Subsurface and Atmospheric Influences on Solar Activity CS-383, Astron. Soc. Pacific, San Francisco, 221. ADS.

Hurford, G.J., Krucker, S., Lin, R.P., Schwartz, R.A., Share, G.H., Smith, D.M.: 2006, Gamma-ray imaging of the 2003 October/November solar flares. Astrophys. J. Lett. 644, L93. DOI. ADS.

Innes, D.E., Inhester, B., Axford, W.I., Wilhelm, K.: 1997, Bi-directional plasma jets produced by magnetic reconnection on the Sun. Nature 386, 811. DOI. ADS.

Innes, D.E., Guo, L.-J., Huang, Y.-M., Bhattacharjee, A.: 2015, IRIS Si IV line profiles: an indication for the plasmoid instability during small-scale magnetic reconnection on the Sun. Astrophys. J. 813, 86. DOI. ADS.

Ishikawa, R.T., Katsukawa, Y., Oba, T., Nakata, M., Nagaoka, K., Kobayashi, T.: 2020, Study of the dynamics of convective turbulence in the solar granulation by spectral line broadening and asymmetry. Astrophys. J. 890, 138. DOI. ADS. 
Isobe, H., Tripathi, D., Archontis, V.: 2007, Ellerman bombs and jets associated with resistive flux emergence. Astrophys. J. Lett. 657, L53. DOI. ADS.

Isobe, H., Yokoyama, T., Shimojo, M., Morimoto, T., Kozu, H., Eto, S., Narukage, N., Shibata, K.: 2002, Reconnection rate in the decay phase of a long duration event flare on 1997 May 12. Astrophys. J. 566, 528. DOI. ADS.

Isobe, H., Kubo, M., Minoshima, T., Ichimoto, K., Katsukawa, Y., Tarbell, T.D., Tsuneta, S., Berger, T.E., Lites, B., Nagata, S., Shimizu, T., Shine, R.A., Suematsu, Y., Title, A.M.: 2007, Flare ribbons observed with G-band and FeI 6302 A, filters of the Solar Optical Telescope on Board Hinode. Publ. Astron. Soc. Japan 59, S807. DOI. ADS.

Hinode Review Team, Al-Janabi, K., Antolin, P., Baker, D., Bellot Rubio, L.R., Bradley, L., Brooks, D.H., Centeno, R., Culhane, J.L., Del Zanna, G., Doschek, G.A., Fletcher, L., Hara, H., Harra, L.K., Hillier, A.S., Imada, S., Klimchuk, J.A., Mariska, J.T., Pereira, T.M.D., Reeves, K.K., Sakao, T., Sakurai, T., Shimizu, T., Shimojo, M., Shiota, D., Solanki, S.K., Sterling, A.C., Su, Y., Suematsu, Y., Tarbell, T.D., Tiwari, S.K., Toriumi, S., Ugarte-Urra, I., Warren, H.P., Watanabe, T., Young, P.R.: 2019, Achievements of Hinode in the first eleven years. Publ. Astron. Soc. Japan 71, R1. DOI. ADS.

Jacques, S.A.: 1977, Momentum and energy transport by waves in the solar atmosphere and solar wind. Astrophys. J. 215, 942. DOI. ADS.

Janardhan, P., Fujiki, K., Ingale, M., Bisoi, S.K., Rout, D.: 2018, Solar cycle 24: an unusual polar field reversal. Astron. Astrophys. 618, A148. DOI. ADS.

Jaeggli, S., et al.: 2021, The DKIST Diffraction-Limited Near-Infrared Spectropolarimeter (DL-NIRSP). Solar Phys. 296 (in preparation).

Janvier, M., Aulanier, G., Bommier, V., Schmieder, B., Démoulin, P., Pariat, E.: 2014, Electric currents in flare ribbons: observations and three-dimensional standard model. Astrophys. J. 788, 60. DOI. ADS.

Jefferies, S.M., McIntosh, S.W., Armstrong, J.D., Bogdan, T.J., Cacciani, A.r., Fleck, B.: 2006, Magnetoacoustic portals and the basal heating of the solar chromosphere. Astrophys. J. Lett. 648, L151. DOI. ADS.

Jenkins, E.B.: 2013, The fractional ionization of the warm neutral interstellar medium. Astrophys. J. 764, 25. DOI. ADS.

Jess, D.B., Mathioudakis, M., Erdélyi, R., Crockett, P.J., Keenan, F.P., Christian, D.J.: 2009, Alfvén waves in the lower solar atmosphere. Science 323, 1582. DOI. ADS.

Jess, D.B., Pascoe, D.J., Christian, D.J., Mathioudakis, M., Keys, P.H., Keenan, F.P.: 2012, The origin of type I spicule oscillations. Astrophys. J. Lett. 744, L5. DOI. ADS.

Jess, D.B., Morton, R.J., Verth, G., Fedun, V., Grant, S.D.T., Giagkiozis, I.: 2015, Multiwavelength studies of MHD waves in the solar chromosphere. An overview of recent results. Space Sci. Rev. 190, 103. DOI. ADS.

Jess, D.B., Snow, B., Houston, S.J., Botha, G.J.J., Fleck, B., Krishna Prasad, S., Asensio Ramos, A., Morton, R.J., Keys, P.H., Jafarzadeh, S., Stangalini, M., Grant, S.D.T., Christian, D.J.: 2020, A chromospheric resonance cavity in a sunspot mapped with seismology. Nat. Astron. 4, 220. DOI. ADS.

Ji, H., Daughton, W.: 2011, Phase diagram for magnetic reconnection in heliophysical, astrophysical, and laboratory plasmas. Phys. Plasmas 18, 111207. DOI. ADS.

Jing, J., Yuan, Y., Reardon, K., Wiegelmann, T., Xu, Y., Wang, H.: 2011, Nonpotentiality of chromospheric fibrils in NOAA active regions 11092 and 9661. Astrophys. J. 739, 67. DOI. ADS.

Jing, J., Xu, Y., Cao, W., Liu, C., Gary, D., Wang, H.: 2016, Unprecedented fine structure of a solar flare revealed by the $1.6 \mathrm{~m}$ New Solar Telescope. Sci. Rep. 6, 24319. DOI. ADS.

Jones, G.H., Knight, M.M., Battams, K., Boice, D.C., Brown, J., Giordano, S., Raymond, J., Snodgrass, C., Steckloff, J.K., Weissman, P., Fitzsimmons, A., Lisse, C., Opitom, C., Birkett, K.S., Bzowski, M., Decock, A., Mann, I., Ramanjooloo, Y., McCauley, P.: 2018, The science of sungrazers, sunskirters, and other near-Sun comets. Space Sci. Rev. 214, 20. DOI. ADS.

Judge, P.G., Reardon, K., Cauzzi, G.: 2012, Evidence for sheet-like elementary structures in the Sun's atmosphere? Astrophys. J. Lett. 755, L11. DOI. ADS.

Judge, P.G., Tritschler, A., Low, B.C.: 2011, Thermal fine structure and magnetic fields in the solar atmosphere: spicules and fibrils. Astrophys. J. Lett. 730, L4. DOI. ADS.

Kaimal, J.C., Wyngaard, J.C., Haugen, D.A., Coté, O.R., Izumi, Y., Caughey, S.J., Readings, C.J.: 1976, Turbulence structure in the convective boundary layer. J. Atmos. Sci. 33, 2152. DOI. ADS.

Kalkofen, W.: 1997, Oscillations in chromospheric network bright points. Astrophys. J. Lett. 486, L145. DOI. ADS.

Karpen, J.T., Antiochos, S.K., DeVore, C.R.: 2012, The mechanisms for the onset and explosive eruption of coronal mass ejections and eruptive flares. Astrophys. J. 760, 81. DOI. ADS.

Kasper, J.C., Stevens, M.L., Lazarus, A.J., Steinberg, J.T., Ogilvie, K.W.: 2007, Solar wind helium abundance as a function of speed and heliographic latitude: variation through a solar cycle. Astrophys. J. 660, 901. DOI. ADS. 
Kasper, J.C., Stevens, M.L., Korreck, K.E., Maruca, B.A., Kiefer, K.K., Schwadron, N.A., Lepri, S.T.: 2012, Evolution of the relationships between helium abundance, minor ion charge state, and solar wind speed over the solar cycle. Astrophys. J. 745, 162. DOI. ADS.

Kasper, J.C., Bale, S.D., Belcher, J.W., Berthomier, M., Case, A.W., Chandran, B.D.G., Curtis, D.W., Gallagher, D., Gary, S.P., Golub, L., Halekas, J.S., Ho, G.C., Horbury, T.S., Hu, Q., Huang, J., Klein, K.G., Korreck, K.E., Larson, D.E., Livi, R., Maruca, B., Lavraud, B., Louarn, P., Maksimovic, M., Martinovic, M., McGinnis, D., Pogorelov, N.V., Richardson, J.D., Skoug, R.M., Steinberg, J.T., Stevens, M.L., Szabo, A., Velli, M., Whittlesey, P.L., Wright, K.H., Zank, G.P., MacDowall, R.J., McComas, D.J., McNutt, R.L., Pulupa, M., Raouafi, N.E., Schwadron, N.A.: 2019, Alfvénic velocity spikes and rotational flows in the near-Sun solar wind. Nature 576, 228. DOI. ADS.

Katsukawa, Y., Berger, T.E., Ichimoto, K., Lites, B.W., Nagata, S., Shimizu, T., Shine, R.A., Suematsu, Y., Tarbell, T.D., Title, A.M., Tsuneta, S.: 2007, Small-scale jetlike features in penumbral chromospheres. Science 318, 1594. DOI. ADS.

Kawabata, Y., Iida, Y., Doi, T., Akiyama, S., Yashiro, S., Shimizu, T.: 2018, Statistical relation between solar flares and coronal mass ejections with respect to sigmoidal structures in active regions. Astrophys. J. 869, 99. DOI. ADS.

Kawaguchi, I.: 1970, Observed interaction between prominences. Publ. Astron. Soc. Japan 22, 405. ADS.

Kazachenko, M.D., Fisher, G.H., Welsch, B.T.: 2014, A comprehensive method of estimating electric fields from vector magnetic field and Doppler measurements. Astrophys. J. 795, 17. DOI. ADS.

Kazachenko, M.D., Fisher, G.H., Welsch, B.T., Liu, Y., Sun, X.: 2015, Photospheric electric fields and energy fluxes in the eruptive active region NOAA 11158. Astrophys. J. 811, 16. DOI. ADS.

Kerr, G.S., Fletcher, L.: 2014, Physical properties of white-light sources in the 2011 February 15 solar flare. Astrophys. J. 783, 98. DOI. ADS.

Kerr, G.S., Fletcher, L., Russell, A.J.B., Allred, J.C.: 2016, Simulations of the Mg II k and Ca II 8542 lines from an Alfvén wave-heated flare chromosphere. Astrophys. J. 827, 101. DOI. ADS.

Keys, P.H., Morton, R.J., Jess, D.B., Verth, G., Grant, S.D.T., Mathioudakis, M., Mackay, D.H., Doyle, J.G., Christian, D.J., Keenan, F.P., Erdélyi, R.: 2018, Photospheric observations of surface and body modes in solar magnetic pores. Astrophys. J. 857, 28. DOI. ADS.

Khomenko, E.: 2017, On the effects of ion-neutral interactions in solar plasmas. Plasma Phys. Control. Fusion 59, 014038. DOI. ADS.

Khomenko, E., Collados, M.: 2012, Heating of the magnetized solar chromosphere by partial ionization effects. Astrophys. J. 747, 87. DOI. ADS.

Khomenko, E., Centeno, R., Collados, M., Trujillo Bueno, J.: 2008, Channeling 5 minute photospheric oscillations into the solar outer atmosphere through small-scale vertical magnetic flux tubes. Astrophys. J. Lett. 676, L85. DOI. ADS.

Khomenko, E., Martínez Pillet, V., Solanki, S.K., del Toro Iniesta, J.C., Gandorfer, A., Bonet, J.A., Domingo, V., Schmidt, W., Barthol, P., Knölker, M.: 2010, Where the granular flows bend. Astrophys. J. Lett. 723, L159. DOI. ADS.

Khomenko, E., Vitas, N., Collados, M., de Vicente, A.: 2017, Numerical simulations of quiet Sun magnetic fields seeded by the Biermann battery. Astron. Astrophys. 604, A66. DOI. ADS.

Khomenko, E., Vitas, N., Collados, M., de Vicente, A.: 2018, Three-dimensional simulations of solar magneto-convection including effects of partial ionization. Astron. Astrophys. 618, A87. DOI. ADS.

Kiefer, F., Lecavelier des Etangs, A., Boissier, J., Vidal-Madjar, A., Beust, H., Lagrange, A.-M., Hébrard, G., Ferlet, R.: 2014, Two families of exocomets in the $\beta$ Pictoris system. Nature 514, 462. DOI. ADS.

Kilpua, E.K.J., Lugaz, N., Mays, M.L., Temmer, M.: 2019, Forecasting the structure and orientation of earthbound coronal mass ejections. Space Weather 17, 498. DOI. ADS.

Kim, Y.-H., Bong, S.-C., Park, Y.-D., Cho, K.-S., Moon, Y.-J.: 2009, Near-simultaneous observations of X-ray plasma ejection, coronal mass ejection, and type II radio burst. Astrophys. J. 705, 1721. DOI. ADS.

Kiss, T.S., Erdélyi, R.: 2018, On quasi-biennial oscillations in chromospheric macrospicules and their potential relation to the global solar magnetic field. Astrophys. J. 857, 113. DOI. ADS.

Kiss, T.S., Gyenge, N., Erdélyi, R.: 2017, Systematic variations of macrospicule properties observed by SDO/AIA over half a decade. Astrophys. J. 835, 47. DOI. ADS.

Kitiashvili, I.N., Kosovichev, A.G., Mansour, N.N., Lele, S.K., Wray, A.A.: 2012, Vortex tubes of turbulent solar convection. Phys. Scr. 86, 018403. DOI. ADS.

Kleint, L.: 2017, First detection of chromospheric magnetic field changes during an X1-flare. Astrophys. J. 834, 26. DOI. ADS.

Kleint, L., Berdyugina, S.V., Shapiro, A.I., Bianda, M.: 2010, Solar turbulent magnetic fields: surprisingly homogeneous distribution during the solar minimum. Astron. Astrophys. 524, A37. DOI. ADS.

Kleint, L., Shapiro, A.I., Berdyugina, S.V., Bianda, M.: 2011, Solar turbulent magnetic fields: non-LTE modeling of the Hanle effect in the $\mathrm{C}_{2}$ molecule. Astron. Astrophys. 536, A47. DOI. ADS. 
Kleint, L., Heinzel, P., Judge, P., Krucker, S.: 2016, Continuum enhancements in the ultraviolet, the visible and the infrared during the X1 flare on 2014 March 29. Astrophys. J. 816, 88. DOI. ADS.

Klimchuk, J.A.: 2000, Cross-sectional properties of coronal loops. Solar Phys. 193, 53. DOI. ADS.

Klimchuk, J.A.: 2006, On solving the coronal heating problem. Solar Phys. 234, 41. DOI. ADS.

Klimchuk, J.A.: 2012, The role of type II spicules in the upper solar atmosphere. J. Geophys. Res. Space Phys. 117, A12102. DOI. ADS.

Klimchuk, J.A.: 2015, Key aspects of coronal heating. Phil. Trans. Roy. Soc. London Ser. A 373, 20140256. DOI. ADS.

Klinger, J., Espinasse, S., Schmidt, B.: 1989, Some considerations on cohesive forces in sun-grazing comets. In: Hunt, J.J., Guyenne, T.D. (eds.) Physics and Mechanics of Cometary Materials CS-302, ESA, Noordwijk, 197. ADS.

Knizhnik, K.J., Antiochos, S.K., DeVore, C.R.: 2017, The role of magnetic helicity in structuring the solar corona. Astrophys. J. 835, 85. DOI. ADS.

Kohutova, P., Verwichte, E.: 2016, Analysis of coronal rain observed by IRIS, HINODE/SOT, and SDO/AIA: transverse oscillations, kinematics, and thermal evolution. Astrophys. J. 827, 39. DOI. ADS.

Kondrashova, N.N.: 2016, Spectropolarimetric investigation of an Ellerman bomb: 1. Observations. Kinemat. Phys. Celest. Bodies 32, 13. DOI. ADS.

Kontogiannis, I., Georgoulis, M.K., Guerra, J.A., Park, S.-H., Bloomfield, D.S.: 2019, Which photospheric characteristics are most relevant to active-region coronal mass ejections? Solar Phys. 294, 130. DOI. ADS.

Korsós, M.B., Romano, P., Morgan, H., Ye, Y., Erdélyi, R., Zuccarello, F.: 2020a, Differences in periodic magnetic helicity injection behavior between flaring and non-flaring active regions: case study. Astrophys. J. Lett. 897, L23. DOI. ADS.

Korsós, M.B., Georgoulis, M.K., Gyenge, N., Bisoi, S.K., Yu, S., Poedts, S., Nelson, C.J., Liu, J., Yan, Y., Erdélyi, R.: 2020b, Solar flare prediction using magnetic field diagnostics above the photosphere. Astrophys. J. 896, 119. DOI. ADS.

Kosovichev, A.G., Zharkova, V.V.: 1998, X-ray flare sparks quake inside Sun. Nature 393, 317. DOI. ADS.

Kostik, R., Khomenko, E.: 2013, Properties of oscillatory motions in a facular region. Astron. Astrophys. 559, A107. DOI. ADS.

Kowalski, A.F., Hawley, S.L., Carlsson, M., Allred, J.C., Uitenbroek, H., Osten, R.A., Holman, G.: 2015, New insights into white-light flare emission from radiative-hydrodynamic modeling of a chromospheric condensation. Solar Phys. 290, 3487. DOI. ADS.

Kowalski, A.F., Allred, J.C., Uitenbroek, H., Tremblay, P.-E., Brown, S., Carlsson, M., Osten, R.A., Wisniewski, J.P., Hawley, S.L.: 2017a, Hydrogen Balmer line broadening in solar and stellar flares. Astrophys. J. 837, 125. DOI. ADS.

Kowalski, A.F., Allred, J.C., Daw, A., Cauzzi, G., Carlsson, M.: 2017b, The atmospheric response to high nonthermal electron beam fluxes in solar flares. I. Modeling the brightest NUV footpoints in the X1 solar flare of 2014 March 29. Astrophys. J. 836, 12. DOI. ADS.

Krivova, N.A., Solanki, S.K., Fligge, M., Unruh, Y.C.: 2003, Reconstruction of solar irradiance variations in cycle 23: is solar surface magnetism the cause? Astron. Astrophys. 399, L1. DOI. ADS.

Krucker, S., Battaglia, M.: 2014, Particle densities within the acceleration region of a solar flare. Astrophys. J. 780, 107. DOI. ADS.

Krucker, S., Hudson, H.S., Glesener, L., White, S.M., Masuda, S., Wuelser, J.-P., Lin, R.P.: 2010, Measurements of the coronal acceleration region of a solar flare. Astrophys. J. 714, 1108. DOI. ADS.

Krucker, S., Hudson, H.S., Jeffrey, N.L.S., Battaglia, M., Kontar, E.P., Benz, A.O., Csillaghy, A., Lin, R.P.: 2011, High-resolution imaging of solar flare ribbons and its implication on the thick-target beam model. Astrophys. J. 739, 96. DOI. ADS.

Kubo, M., Shimizu, T., Tsuneta, S.: 2007, Vector magnetic fields of moving magnetic features and flux removal from a sunspot. Astrophys. J. 659, 812. DOI. ADS.

Kuckein, C., Martínez Pillet, V., Centeno, R.: 2012, An active region filament studied simultaneously in the chromosphere and photosphere. I. Magnetic structure. Astron. Astrophys. 539, A131. DOI. ADS.

Kuhn, J.R., Penn, M.J., Mann, I.: 1996, The near-infrared coronal spectrum. Astrophys. J. Lett. 456 , L67. DOI. ADS.

Kuhn, J.R., Arnaud, J., Jaeggli, S., Lin, H., Moise, E.: 2007, Detection of an extended near-Sun neutral helium cloud from ground-based infrared coronagraph spectropolarimetry. Astrophys. J. Lett. 667, L203. DOI. ADS.

Kuperus, M., Ionson, J.A., Spicer, D.S.: 1981, On the theory of coronal heating mechanisms. Annu. Rev. Astron. Astrophys. 19, 7. DOI. ADS.

Kuridze, D., Mathioudakis, M., Morgan, H., Oliver, R., Kleint, L., Zaqarashvili, T.V., Reid, A., Koza, J., Löfdahl, M.G., Hillberg, T., Kukhianidze, V., Hanslmeier, A.: 2019, Mapping the magnetic field of flare coronal loops. Astrophys. J. 874, 126. DOI. ADS. 
Kurokawa, H., Kawaguchi, I., Funakoshi, Y., Nakai, Y.: 1982, Morphological and evolutional features of Ellerman bombs. Solar Phys. 79, 77. DOI. ADS.

Kurokawa, H., Hanaoka, Y., Shibata, K., Uchida, Y.: 1987, Rotating eruption of an untwisting filament triggered by the 3B flare of 25 April, 1984. Solar Phys. 108, 251. DOI. ADS.

Kusano, K., Bamba, Y., Yamamoto, T.T., Iida, Y., Toriumi, S., Asai, A.: 2012, Magnetic field structures triggering solar flares and coronal mass ejections. Astrophys. J. 760, 31. DOI. ADS.

Lamb, D.A.: 2017, Measurements of solar differential rotation and meridional circulation from tracking of photospheric magnetic features. Astrophys. J. 836, 10. DOI. ADS.

Laming, J.M.: 2015, The FIP and inverse FIP effects in solar and stellar coronae. Liv. Rev. Solar Phys. 12, 2. DOI. ADS.

Laming, J.M., Feldman, U.: 2001, The solar helium abundance in the outer corona determined from observations with SUMER/SOHO. Astrophys. J. 546, 552. DOI. ADS.

Laming, J.M., Feldman, U.: 2003, The variability of the solar coronal helium abundance: polar coronal holes compared to the quiet Sun. Astrophys. J. 591, 1257. DOI. ADS.

Landi, E., Habbal, S.R., Tomczyk, S.: 2016, Coronal plasma diagnostics from ground-based observations. $J$. Geophys. Res. Space Phys. 121, 8237. DOI. ADS.

Landi, E., Gruesbeck, J.R., Lepri, S.T., Zurbuchen, T.H.: 2012, New solar wind diagnostic using both in situ and spectroscopic measurements. Astrophys. J. 750, 159. DOI. ADS.

Lanzerotti, L.J., Uberoi, C.: 1988, Comment on "MHD wave breaking in the outer plasmasphere". Geophys. Res. Lett. 15, 471. DOI. ADS.

Leake, J.E., Lukin, V.S., Linton, M.G., Meier, E.T.: 2012, Multi-fluid simulations of chromospheric magnetic reconnection in a weakly ionized reacting plasma. Astrophys. J. 760, 109. DOI. ADS.

Leenaarts, J., de la Cruz Rodríguez, J., Danilovic, S., Scharmer, G., Carlsson, M.: 2018, Chromospheric heating during flux emergence in the solar atmosphere. Astron. Astrophys. 612, A28. DOI. ADS.

Leighton, R.B.: 1960, Comments. In: Thomas, R.N. (ed.) Aerodynamic Phenomena in Stellar Atmospheres, IAU Symp. 12, Academic Press, London, 321. ADS.

Leighton, R.B., Noyes, R.W., Simon, G.W.: 1962, Velocity fields in the solar atmosphere. I. Preliminary report. Astrophys. J. 135, 474. DOI. ADS.

Leka, K.D., Canfield, R.C., McClymont, A.N., van Driel-Gesztelyi, L.: 1996, Evidence for current-carrying emerging flux. Astrophys. J. 462, 547. DOI. ADS.

Lenschow, D.H., Boba Stankov, B.: 1986, Length scales in the convective boundary layer. J. Atmos. Sci. 43, 1198. DOI. ADS.

Leroy, J.L., Bommier, V., Sahal-Brechot, S.: 1983, The magnetic field in the prominences of the polar crown. Solar Phys. 83, 135. DOI. ADS.

Levens, P.J., Schmieder, B., López Ariste, A., Labrosse, N., Dalmasse, K., Gelly, B.: 2016a, Magnetic field in atypical prominence structures: bubble, tornado, and eruption. Astrophys. J. 826, 164. DOI. ADS.

Levens, P.J., Schmieder, B., Labrosse, N., López Ariste, A.: 2016b, Structure of prominence legs: plasma and magnetic field. Astrophys. J. 818, 31. DOI. ADS.

Li, L.P., Peter, H.: 2019, Plasma injection into a solar coronal loop. Astron. Astrophys. 626, A98. DOI. ADS.

Li, Y., Xue, J.C., Ding, M.D., Cheng, X., Su, Y., Feng, L., Hong, J., Li, H., Gan, W.Q.: 2018, Spectroscopic observations of a current sheet in a solar flare. Astrophys. J. Lett. 853, L15. DOI. ADS.

Liang, Z.-C., Gizon, L., Schunker, H., Philippe, T.: 2013, Helioseismology of sunspots: defocusing, folding, and healing of wavefronts. Astron. Astrophys. 558, A129. DOI. ADS.

Libbrecht, K.G., Woodard, M.F.: 1990, Solar-cycle effects on solar oscillation frequencies. Nature 345, 779. DOI. ADS.

Libbrecht, T., Joshi, J., Rodríguez, J.d.1.C., Leenaarts, J., Ramos, A.A.: 2017, Observations of Ellerman bomb emission features in $\mathrm{He} \mathrm{I} \mathrm{D}_{3}$ and He I $10830 \AA$ A. Astron. Astrophys. 598, A33. DOI. ADS.

Lighthill, M.J.: 1952, On sound generated aerodynamically. I. General theory. Proc. Roy. Soc. London Ser. A 211, 564. DOI. ADS.

Lin, J., Forbes, T.G.: 2000, Effects of reconnection on the coronal mass ejection process. J. Geophys. Res. 105, 2375. DOI. ADS.

Lin, J., Cranmer, S.R., Farrugia, C.J.: 2008, Plasmoids in reconnecting current sheets: solar and terrestrial contexts compared. J. Geophys. Res. Space Phys. 113, A11107. DOI. ADS.

Lin, J., Li, J., Forbes, T.G., Ko, Y.-K., Raymond, J.C., Vourlidas, A.: 2007, Features and properties of coronal mass ejection/flare current sheets. Astrophys. J. Lett. 658, L123. DOI. ADS.

Linan, L., Pariat, É., Moraitis, K., Valori, G., Leake, J.: 2018, Time variations of the nonpotential and volumethreading magnetic helicities. Astrophys. J. 865, 52. DOI. ADS.

Lindsey, C., Donea, A.-C., Martínez Oliveros, J.C., Hudson, H.S.: 2014, The role of magnetic fields in transient seismic emission driven by atmospheric heating in flares. Solar Phys. 289, 1457. DOI. ADS.

Lingam, M., Hirvijoki, E., Pfefferlé, D., Comisso, L., Bhattacharjee, A.: 2017, Nonlinear resistivity for magnetohydrodynamical models. Phys. Plasmas 24, 042120. DOI. ADS. 
Linker, J.A., Caplan, R.M., Downs, C., Riley, P., Mikic, Z., Lionello, R., Henney, C.J., Arge, C.N., Liu, Y., Derosa, M.L., Yeates, A., Owens, M.J.: 2017, The open flux problem. Astrophys. J. 848, 70. DOI. ADS.

Linsky, J.L., France, K., Ayres, T.: 2013, Computing intrinsic LY $\alpha$ fluxes of F5 V to M5 V stars. Astrophys. J. 766, 69. DOI. ADS.

Lipartito, I., Judge, P.G., Reardon, K., Cauzzi, G.: 2014, The solar chromosphere observed at $1 \mathrm{~Hz}$ and 0." 2 resolution. Astrophys. J. 785, 109. DOI. ADS.

Lites, B.W.: 2011, Hinode observations suggesting the presence of a local small-scale turbulent dynamo. Astrophys. J. 737, 52. DOI. ADS.

Lites, B.W., Kubo, M., Socas-Navarro, H., Berger, T., Frank, Z., Shine, R., Tarbell, T., Title, A., Ichimoto, K., Katsukawa, Y., Tsuneta, S., Suematsu, Y., Shimizu, T., Nagata, S.: 2008, The horizontal magnetic flux of the quiet-Sun internetwork as observed with the Hinode spectro-polarimeter. Astrophys. J. 672, 1237. DOI. ADS.

Liu, C., Cao, W., Chae, J., Ahn, K., Prasad Choudhary, D., Lee, J., Liu, R., Deng, N., Wang, J., Wang, H.: 2018, Evolution of photospheric vector magnetic field associated with moving flare ribbons as seen by GST. Astrophys. J. 869, 21. DOI. ADS.

Liu, J., Nelson, C.J., Erdélyi, R.: 2019, Automated Swirl Detection Algorithm (ASDA) and its application to simulation and observational data. Astrophys. J. 872, 22. DOI. ADS.

Liu, J., Carlsson, M., Nelson, C.J., Erdélyi, R.: 2019a, Co-spatial velocity and magnetic swirls in the simulated solar photosphere. Astron. Astrophys. 632, A97. DOI. ADS.

Liu, J., Nelson, C.J., Snow, B., Wang, Y., Erdélyi, R.: 2019b, Evidence of ubiquitous Alfvén pulses transporting energy from the photosphere to the upper chromosphere. Nat. Commun. 10, 3504. DOI. ADS.

Liu, R.: 2013, Dynamical processes at the vertical current sheet behind an erupting flux rope. Mon. Not. Roy. Astron. Soc. 434, 1309. DOI. ADS.

Löhner-Böttcher, J., Schmidt, W., Schlichenmaier, R., Doerr, H.-P., Steinmetz, T., Holzwarth, R.: 2018, Absolute velocity measurements in sunspot umbrae. Astron. Astrophys. 617, A19. DOI. ADS.

Longcope, D., Qiu, J., Brewer, J.: 2016, A reconnection-driven model of the hard X-ray loop-top source from flare 2004-Feb-26. Astrophys. J. 833, 211. DOI. ADS.

Longcope, D.W.: 2014, A simple model of chromospheric evaporation and condensation driven conductively in a solar flare. Astrophys. J. 795, 10. DOI. ADS.

Longcope, D.W., Beveridge, C.: 2007, A quantitative, topological model of reconnection and flux rope formation in a two-ribbon flare. Astrophys. J. 669, 621. DOI. ADS.

Longcope, D.W., Tarr, L.A.: 2015, Relating magnetic reconnection to coronal heating. Phil. Trans. Roy. Soc. London Ser. A 373, 20140263. DOI. ADS.

Longcope, D., Unverferth, J., Klein, C., McCarthy, M., Priest, E.: 2018, Evidence for downflows in the narrow plasma sheet of 2017 September 10 and their significance for flare reconnection. Astrophys. J. 868, 148. DOI. ADS.

López Fuentes, M.C., Klimchuk, J.A., Démoulin, P.: 2006, The magnetic structure of coronal loops observed by TRACE. Astrophys. J. 639, 459. DOI. ADS.

Lord, J.W., Cameron, R.H., Rast, M.P., Rempel, M., Roudier, T.: 2014, The role of subsurface flows in solar surface convection: modeling the spectrum of supergranular and larger scale flows. Astrophys. J. 793, 24. DOI. ADS.

Loughhead, R.E., Bray, R.J.: 1959, 'Turbulence' and the photospheric granulation. Nature 183, 240. DOI. ADS.

Louis, R.E., Beck, C., Ichimoto, K.: 2014, Small-scale chromospheric jets above a sunspot light bridge. Astron. Astrophys. 567, A96. DOI. ADS.

Louis, R.E., Bayanna, A.R., Mathew, S.K., Venkatakrishnan, P.: 2008, Dynamics of sunspot light bridges as revealed by high-resolution images from Hinode. Solar Phys. 252, 43. DOI. ADS.

Louis, R.E., Bellot Rubio, L.R., Mathew, S.K., Venkatakrishnan, P.: 2011, Supersonic downflows at the umbra-penumbra boundary of sunspots. Astrophys. J. 727, 49. DOI. ADS.

Loureiro, N.F., Schekochihin, A.A., Cowley, S.C.: 2007, Instability of current sheets and formation of plasmoid chains. Phys. Plasmas 14, 100703. DOI. ADS.

Low, B.C.: 1996, Solar activity and the corona. Solar Phys. 167, 217. DOI. ADS.

Ma, L., Zhou, W., Zhou, G., Zhang, J.: 2015, The evolution of arch filament systems and moving magnetic features around a sunspot. Astron. Astrophys. 583, A110. DOI. ADS.

Mackay, D.H., DeVore, C.R., Antiochos, S.K., Yeates, A.R.: 2018, Magnetic helicity condensation and the solar cycle. Astrophys. J. 869, 62. DOI. ADS.

Markhotok, A.: 2018, A shock wave instability induced on a periodically disturbed interface with plasma. IEEE Trans. Plasma Sci. 46, 2821. DOI. ADS.

Martin, S.F.: 1980, Preflare conditions, changes and events. Solar Phys. 68, 217. DOI. ADS.

Martínez-Gómez, D., Oliver, R., Khomenko, E., Collados, M.: 2020, Two-dimensional simulations of coronal rain dynamics. I. Model consisting of a vertical magnetic field and an unbounded atmosphere. Astron. Astrophys. 634, A36. DOI. ADS. 
Martínez Oliveros, J.C., Couvidat, S., Schou, J., Krucker, S., Lindsey, C., Hudson, H.S., Scherrer, P.: 2011, Imaging spectroscopy of a white-light solar flare. Solar Phys. 269, 269. DOI. ADS.

Martínez Oliveros, J.-C., Hudson, H.S., Hurford, G.J., Krucker, S., Lin, R.P., Lindsey, C., Couvidat, S., Schou, J., Thompson, W.T.: 2012, The height of a white-light flare and its hard X-ray sources. Astrophys. J. Lett. 753, L26. DOI. ADS.

Martínez Pillet, V., Del Toro Iniesta, J.C., Álvarez-Herrero, A., Domingo, V., Bonet, J.A., González Fernández, L., López Jiménez, A., Pastor, C., Gasent Blesa, J.L., Mellado, P., Piqueras, J., Aparicio, B., Balaguer, M., Ballesteros, E., Belenguer, T., Bellot Rubio, L.R., Berkefeld, T., Collados, M., Deutsch, W., Feller, A., Girela, F., Grauf, B., Heredero, R.L., Herranz, M., Jerónimo, J.M., Laguna, H., Meller, R., Menéndez, M., Morales, R., Orozco Suárez, D., Ramos, G., Reina, M., Ramos, J.L., Rodríguez, P., Sánchez, A., Uribe-Patarroyo, N., Barthol, P., Gandorfer, A., Knoelker, M., Schmidt, W., Solanki, S.K., Vargas Domínguez, S.: 2011, The Imaging Magnetograph eXperiment (IMaX) for the Sunrise balloon-borne solar observatory. Solar Phys. 268, 57. DOI. ADS.

Martinez Pillet, V., Tritschler, A., Harra, L., Andretta, V., Vourlidas, A., Raouafi, N., Alterman, B.L., Bellot Rubio, L., Cauzzi, G., Cranmer, S.R., Gibson, S., Habbal, S., Ko, Y.K., Lepri, S.T., Linker, J., Malaspina, D.M., Matthews, S., Parenti, S., Petrie, G., Spadaro, D., Ugarte-Urra, I., Warren, H., Winslow, R.: 2020, Solar physics in the 2020s: DKIST, Parker Solar Probe, and solar orbiter as a multi-messenger constellation. ADS. arXiv.

Martínez-Sykora, J., De Pontieu, B., Hansteen, V.: 2012, Two-dimensional radiative magnetohydrodynamic simulations of the importance of partial ionization in the chromosphere. Astrophys. J. 753, 161. DOI. ADS.

Martínez-Sykora, J., De Pontieu, B., Carlsson, M., Hansteen, V.: 2016, On the misalignment between chromospheric features and the magnetic field on the Sun. Astrophys. J. Lett. 831, L1. DOI. ADS.

Martínez-Sykora, J., De Pontieu, B., Hansteen, V.H., Rouppe van der Voort, L., Carlsson, M., Pereira, T.M.D.: 2017, On the generation of solar spicules and Alfvénic waves. Science 356, 1269. DOI. ADS.

Martínez-Sykora, J., Hansteen, V.H., Gudiksen, B., Carlsson, M., De Pontieu, B., Gošić, M.: 2019, On the origin of the magnetic energy in the quiet solar chromosphere. Astrophys. J. 878, 40. DOI. ADS.

Martinović, M.M., Klein, K.G., Bourouaine, S.: 2019, Radial evolution of stochastic heating in low- $\beta$ solar wind. Astrophys. J. 879, 43. DOI. ADS.

Martres, M.-J., Soru-Escaut, I., Nakagawa, Y.: 1977, Halpha off-band pre-flare activities. Astron. Astrophys. 59, 255. ADS.

Mason, E.I., Antiochos, S.K., Viall, N.M.: 2019, Observations of solar coronal rain in null point topologies. Astrophys. J. Lett. 874, L33. DOI. ADS.

Mathioudakis, M., Jess, D.B., Erdélyi, R.: 2013, Alfvén waves in the solar atmosphere. From theory to observations. Space Sci. Rev. 175, 1. DOI. ADS.

Matsumoto, T., Kitai, R., Shibata, K., Otsuji, K., Naruse, T., Shiota, D., Takasaki, H.: 2008, Height dependence of gas flows in an Ellerman bomb. Publ. Astron. Soc. Japan 60, 95. DOI. ADS.

McAteer, R.T.J., Gallagher, P.T., Williams, D.R., Mathioudakis, M., Bloomfield, D.S., Phillips, K.J.H., Keenan, F.P.: 2003, Observational evidence for mode coupling in the chromospheric network. Astrophys. J. 587, 806. DOI. ADS.

McCauley, P.I., Saar, S.H., Raymond, J.C., Ko, Y.-K., Saint-Hilaire, P.: 2013, Extreme-ultraviolet and X-ray observations of Comet Lovejoy (C/2011 W3) in the lower corona. Astrophys. J. 768, 161. DOI. ADS.

McClintock, W.E., Bradley, E.T., Vervack, R.J., Killen, R.M., Sprague, A.L., Izenberg, N.R., Solomon, S.C.: 2008, Mercury's exosphere: observations during MESSENGER's first Mercury flyby. Science 321, 92. DOI. ADS.

McClure, R.L., Rast, M.P., Martínez Pillet, V.: 2019, Doppler events in the solar photosphere: the coincident superposition of fast granular flows and p-mode coherence patches. Solar Phys. 294, 18. DOI. ADS.

McIntosh, S.W., Davey, A.R., Hassler, D.M., Armstrong, J.D., Curdt, W., Wilhelm, K., Lin, G.: 2007, Observations supporting the role of magnetoconvection in energy supply to the quiescent solar atmosphere. Astrophys. J. 654, 650. DOI. ADS.

McIntosh, S.W., De Pontieu, B., Carlsson, M., Hansteen, V., Boerner, P., Goossens, M.: 2011a, Alfvénic waves with sufficient energy to power the quiet solar corona and fast solar wind. Nature 475, 477. DOI. ADS.

McIntosh, S.W., Leamon, R.J., Hock, R.A., Rast, M.P., Ulrich, R.K.: 2011b, Observing evolution in the supergranular network length scale during periods of low solar activity. Astrophys. J. Lett. 730, L3. DOI. ADS

Mei, Z., Shen, C., Wu, N., Lin, J., Murphy, N.A., Roussev, I.I.: 2012, Numerical experiments on magnetic reconnection in solar flare and coronal mass ejection current sheets. Mon. Not. Roy. Astron. Soc. 425, 2824. DOI. ADS.

Mendoza-Briceño, C.A., Erdélyi, R., Sigalotti, L.D.G.: 2002, Coronal loop heating by random energy releases. Astrophys. J. Lett. 579, L49. DOI. ADS. 
Mendoza-Briceño, C.A., Sigalotti, L.D.G., Erdélyi, R.: 2005, Catastrophic cooling of impulsively heated coronal loops. Astrophys. J. 624, 1080. DOI. ADS.

Merkel, A.W., Cassidy, T.A., Vervack, R.J., McClintock, W.E., Sarantos, M., Burger, M.H., Killen, R.M.: 2017, Seasonal variations of Mercury's magnesium dayside exosphere from MESSENGER observations. Icarus 281, 46. DOI. ADS.

Meyer, J.-P.: 1985a, Solar-stellar outer atmospheres and energetic particles, and galactic cosmic rays. Astrophys. J. Suppl. Ser. 57, 173. DOI. ADS.

Meyer, J.-P.: 1985b, The baseline composition of solar energetic particles. Astrophys. J. Suppl. Ser. 57, 151. DOI. ADS.

Mghebrishvili, I., Zaqarashvili, T.V., Kukhianidze, V., Ramishvili, G., Shergelashvili, B., Veronig, A., Poedts, S.: 2015, Dynamics of a solar prominence tornado observed by SDO/AIA on 2012 November 7-8. Astrophys. J. 810, 89. DOI. ADS.

Mghebrishvili, I., Zaqarashvili, T.V., Kukhianidze, V., Kuridze, D., Tsiklauri, D., Shergelashvili, B.M., Poedts, S.: 2018, Association between tornadoes and instability of hosting prominences. Astrophys. J. 861, 112. DOI. ADS.

Michalitsanos, A.G.: 1973, The five minute period oscillation in magnetically active regions. Solar Phys. 30, 47. DOI. ADS.

Miesch, M.S.: 2005, Large-scale dynamics of the convection zone and tachocline. Liv. Rev. Solar Phys. 2 , 1. DOI. ADS.

Mignone, A.: 2005, The dynamics of radiative shock waves: linear and nonlinear evolution. Astrophys. J. 626, 373. DOI. ADS.

Miguel, Y., Kaltenegger, L.: 2014, Exploring atmospheres of hot mini-Neptunes and extrasolar giant planets orbiting different stars with application to HD 97658b, WASP-12b, CoRoT-2b, XO-1b, and HD 189733b. Astrophys. J. 780, 166. DOI. ADS.

Mikic, Z., Linker, J.A.: 1994, Disruption of coronal magnetic field arcades. Astrophys. J. 430, 898. DOI. ADS.

Mikuła, K., Heinzel, P., Liu, W., Berlicki, A.: 2017, Structure and dynamics of cool flare loops observed by the Interface Region Imaging Spectrograph. Astrophys. J. 845, 30. DOI. ADS.

Moeng, C.-H., Lemone, M.A., Khairoutdinov, M.F., Krueger, S.K., Bogenschutz, P.A., Randall, D.A.: 2009, The tropical marine boundary layer under a deep convection system: a large-eddy simulation study. $J$. Adv. Model. Earth Syst. 1, 16. DOI. ADS.

Moffatt, H.K.: 1969, The degree of knottedness of tangled vortex lines. J. Fluid Mech. 35, 117. DOI. ADS.

Moffatt, H.K.: 2014, Helicity and singular structures in fluid dynamics. Proc. Natl. Acad. Sci. USA 111, 3663. DOI. ADS.

Moffatt, H.K.: 2016, Helicity and celestial magnetism. Proc. Roy. Soc. London Ser. A 472, 20160183. DOI. ADS.

Moise, E., Raymond, J., Kuhn, J.R.: 2010, Properties of the diffuse neutral helium in the inner heliosphere. Astrophys. J. 722, 1411. DOI. ADS.

Moore, R.L., Sterling, A.C., Cirtain, J.W., Falconer, D.A.: 2011, Solar X-ray jets, type-II spicules, granulesize emerging bipoles, and the genesis of the heliosphere. Astrophys. J. Lett. 731, L18. DOI. ADS.

Moore, T.E., Gallagher, D.L., Horwitz, J.L., Comfort, R.H.: 1987, MHD wave breaking in the outer plasmasphere. Geophys. Res. Lett. 14, 1007. DOI. ADS.

Morton, R.J.: 2012, Chromospheric jets around the edges of sunspots. Astron. Astrophys. 543, A6. DOI. ADS.

Morton, R.J., Weberg, M.J., McLaughlin, J.A.: 2019, A basal contribution from p-modes to the Alfvénic wave flux in the Sun's corona. Nat. Astron. 3, 223. DOI. ADS.

Morton, R.J., Erdélyi, R., Jess, D.B., Mathioudakis, M.: 2011, Observations of sausage modes in magnetic pores. Astrophys. J. Lett. 729, L18. DOI. ADS.

Morton, R.J., Verth, G., Jess, D.B., Kuridze, D., Ruderman, M.S., Mathioudakis, M., Erdélyi, R.: 2012, Observations of ubiquitous compressive waves in the Sun's chromosphere. Nat. Commun. 3, 1315. DOI. ADS.

Moschou, S.P., Keppens, R., Xia, C., Fang, X.: 2015, Simulating coronal condensation dynamics in 3D. Adv. Space Res. 56, 2738. DOI. ADS.

Mozer, F.S., Agapitov, O.V., Bale, S.D., Bonnell, J.W., Case, T., Chaston, C.C., Curtis, D.W., Dudok de Wit, T., Goetz, K., Goodrich, K.A., Harvey, P.R., Kasper, J.C., Korreck, K.E., Krasnoselskikh, V., Larson, D.E., Livi, R., MacDowall, R.J., Malaspina, D., Pulupa, M., Stevens, M., Whittlesey, P.L., Wygant, J.R.: 2020, Switchbacks in the solar magnetic field: their evolution, their content, and their effects on the plasma. Astrophys. J. Suppl. Ser. 246, 68. DOI. ADS.

Muhamad, J., Kusano, K., Inoue, S., Shiota, D.: 2017, Magnetohydrodynamic simulations for studying solar flare trigger mechanism. Astrophys. J. 842, 86. DOI. ADS. 
Müller, D.A.N., Hansteen, V.H., Peter, H.: 2003, Dynamics of solar coronal loops. I. Condensation in cool loops and its effect on transition region lines. Astron. Astrophys. 411, 605. DOI. ADS.

Müller, D., Marsden, R.G., St. Cyr, O.C., Gilbert, H.R.: 2013, Solar orbiter. Exploring the Sun-heliosphere connection. Solar Phys. 285, 25. DOI. ADS.

Müller, D., St. Cyr, O.C., Zouganelis, I., Gilbert, H.R., Marsden, R., Nieves-Chinchilla, T., Antonucci, E., Auchère, F., Berghmans, D., Horbury, T.S., Howard, R.A., Krucker, S., Maksimovic, M., Owen, C.J., Rochus, P., Rodriguez-Pacheco, J., Romoli, M., Solanki, S.K., Bruno, R., Carlsson, M., Fludra, A., Harra, L., Hassler, D.M., Livi, S., Louarn, P., Peter, H., Schühle, U., Teriaca, L., del Toro Iniesta, J.C., Wimmer-Schweingruber, R.F., Marsch, E., Velli, M., De Groof, A., Walsh, A., Williams, D.: 2020, The solar orbiter mission. Science overview. Astron. Astrophys. 642, A1. DOI. ADS.

Murabito, M., Romano, P., Guglielmino, S.L., Zuccarello, F.: 2017, On the formation of a stable penumbra in a region of flux emergence in the Sun. Astrophys. J. 834, 76. DOI. ADS.

Murphy, N.A., Miralles, M.P., Pope, C.L., Raymond, J.C., Winter, H.D., Reeves, K.K., Seaton, D.B., van Ballegooijen, A.A., Lin, J.: 2012, Asymmetric magnetic reconnection in solar flare and coronal mass ejection current sheets. Astrophys. J. 751, 56. DOI. ADS.

Nakariakov, V.M., Verwichte, E.: 2005, Coronal waves and oscillations. Liv. Rev. Solar Phys. 2, 3. DOI. ADS.

Nakariakov, V.M., Ofman, L., Deluca, E.E., Roberts, B., Davila, J.M.: 1999, TRACE observation of damped coronal loop oscillations: implications for coronal heating. Science 285, 862. DOI. ADS.

Neidig, D.F.: 1989, The importance of solar white-light flares. Solar Phys. 121, 261. DOI. ADS.

Neidig, D.F., Wiborg, P.H. Jr.: 1984, The hydrogen emission spectrum in three white light flares. Solar Phys. 92, 217. DOI. ADS.

Neidig, D.F., Kiplinger, A.L., Cohl, H.S., Wiborg, P.H.: 1993, The solar white-light flare of 1989 March 7: simultaneous multiwavelength observations at high time resolution. Astrophys. J. 406, 306. DOI. ADS.

Nelson, C.J., Krishna Prasad, S., Mathioudakis, M.: 2020, Evolution of supersonic downflows in a sunspot. Astron. Astrophys. 636, A35. DOI. ADS.

Nelson, C.J., Freij, N., Reid, A., Oliver, R., Mathioudakis, M., Erdélyi, R.: 2017a, IRIS burst spectra cospatial to a quiet-Sun Ellerman-like brightening. Astrophys. J. 845, 16. DOI. ADS.

Nelson, C.J., Henriques, V.M.J., Mathioudakis, M., Keenan, F.P.: 2017b, The formation of small-scale umbral brightenings in sunspot atmospheres. Astron. Astrophys. 605, A14. DOI. ADS.

Ni, L., Kliem, B., Lin, J., Wu, N.: 2015, Fast magnetic reconnection in the solar chromosphere mediated by the plasmoid instability. Astrophys. J. 799, 79. DOI. ADS.

Ni, L., Ji, H., Murphy, N.A., Jonathan, J.: 2020, Magnetic reconnection in partially ionized plasmas. Proc. Roy. Soc. A 476, 20190867.

Nigam, R., Kosovichev, A.G., Scherrer, P.H., Schou, J.: 1998, Asymmetry in velocity and intensity helioseismic spectra: a solution to a long-standing puzzle. Astrophys. J. Lett. 495, L115. DOI. ADS.

Nishizuka, N., Nakamura, T., Kawate, T., Singh, K.A.P., Shibata, K.: 2011, Statistical study of chromospheric anemone jets observed with Hinode/SOT. Astrophys. J. 731, 43. DOI. ADS.

Nordlund, A.: 1985, Solar convection. Solar Phys. 100, 209. DOI. ADS.

Nordlund, Å., Stein, R.F., Asplund, M.: 2009, Solar surface convection. Liv. Rev. Solar Phys. 6, 2. DOI. ADS.

Nordlund, A., Spruit, H.C., Ludwig, H.-G., Trampedach, R.: 1997, Is stellar granulation turbulence? Astron. Astrophys. 328, 229. ADS.

Okamoto, T.J., Sakurai, T.: 2018, Super-strong magnetic field in sunspots. Astrophys. J. Lett. 852, L16. DOI. ADS.

Okamoto, T.J., Antolin, P., De Pontieu, B., Uitenbroek, H., Van Doorsselaere, T., Yokoyama, T.: 2015, Resonant absorption of transverse oscillations and associated heating in a solar prominence. I. Observational aspects. Astrophys. J. 809, 71. DOI. ADS.

Okunev, O.V., Kneer, F.: 2005, Numerical modeling of solar faculae close to the limb. Astron. Astrophys. 439, 323. DOI. ADS.

Olson, J., Egedal, J., Greess, S., Myers, R., Clark, M., Endrizzi, D., Flanagan, K., Milhone, J., Peterson, E., Wallace, J., Weisberg, D., Forest, C.B.: 2016, Experimental demonstration of the collisionless plasmoid instability below the ion kinetic scale during magnetic reconnection. Phys. Rev. Lett. 116, 255001. DOI. ADS.

O’Malley-James, J.T., Kaltenegger, L.: 2019, Lessons from early Earth: UV surface radiation should not limit the habitability of active M star systems. Mon. Not. Roy. Astron. Soc. 485, 5598. DOI. ADS.

Opik, E.J.: 1966, Sun-grazing comets and tidal disruption. Irish Astron. J. 7, 141. ADS.

Ortiz, A., Hansteen, V.H., Nóbrega-Siverio, D., van der Voort, L.R.: 2020, Ellerman bombs and UV bursts: reconnection at different atmospheric layers. Astron. Astrophys. 633, A58. DOI. ADS.

Owens, M.J., Arge, C.N., Crooker, N.U., Schwadron, N.A., Horbury, T.S.: 2008, Estimating total heliospheric magnetic flux from single-point in situ measurements. J. Geophys. Res. Space Phys. 113, A12103. DOI. ADS. 
Owens, M., Lockwood, M., Macneil, A., Stansby, D.: 2020, Signatures of coronal loop opening via interchange reconnection in the slow solar wind at 1 AU. Solar Phys. 295, 37. DOI. ADS.

Panesar, N.K., Sterling, A.C., Moore, R.L.: 2017, Magnetic flux cancellation as the origin of solar quietregion pre-jet minifilaments. Astrophys. J. 844, 131. DOI. ADS.

Panesar, N.K., Innes, D.E., Tiwari, S.K., Low, B.C.: 2013, A solar tornado triggered by flares? Astron. Astrophys. 549, A105. DOI. ADS.

Panesar, N.K., Sterling, A.C., Moore, R.L., Chakrapani, P.: 2016, Magnetic flux cancelation as the trigger of solar quiet-region coronal jets. Astrophys. J. Lett. 832, L7. DOI. ADS.

Panesar, N.K., Sterling, A.C., Moore, R.L., Tiwari, S.K., De Pontieu, B., Norton, A.A.: 2018, IRIS and SDO observations of solar jetlets resulting from network-edge flux cancelation. Astrophys. J. Lett. 868, L27. DOI. ADS.

Panesar, N.K., Tiwari, S.K., Moore, R.L., Sterling, A.C.: 2020, Network jets as the driver of counterstreaming flows in a solar filament/filament channel. Astrophys. J. Lett. 897, L2. DOI. ADS.

Panos, B., Kleint, L., Huwyler, C., Krucker, S., Melchior, M., Ullmann, D., Voloshynovskiy, S.: 2018, Identifying typical Mg II flare spectra using machine learning. Astrophys. J. 861, 62. DOI. ADS.

Parenti, S.: 2014, Solar prominences: observations. Liv. Rev. Solar Phys. 11, 1. DOI. ADS.

Parenti, S., Bromage, B.J.I., Poletto, G., Noci, G., Raymond, J.C., Bromage, G.E.: 2000, Characteristics of solar coronal streamers. Element abundance, temperature and density from coordinated CDS and UVCS SOHO observations. Astron. Astrophys. 363, 800. ADS.

Pariat, E., Aulanier, G., Schmieder, B., Georgoulis, M.K., Rust, D.M., Bernasconi, P.N.: 2004, Resistive emergence of undulatory flux tubes. Astrophys. J. 614, 1099. DOI. ADS.

Pariat, E., Leake, J.E., Valori, G., Linton, M.G., Zuccarello, F.P., Dalmasse, K.: 2017, Relative magnetic helicity as a diagnostic of solar eruptivity. Astron. Astrophys. 601, A125. DOI. ADS.

Park, S.-H., Tsiropoula, G., Kontogiannis, I., Tziotziou, K., Scullion, E., Doyle, J.G.: 2016, First simultaneous SST/CRISP and IRIS observations of a small-scale quiet Sun vortex. Astron. Astrophys. 586, A25. DOI. ADS.

Park, S.-H., Guerra, J.A., Gallagher, P.T., Georgoulis, M.K., Bloomfield, D.S.: 2018, Photospheric shear flows in solar active regions and their relation to flare occurrence. Solar Phys. 293, 114. DOI. ADS.

Parker, E.N.: 1958, Dynamics of the interplanetary gas and magnetic fields. Astrophys. J. 128, 664. DOI. ADS.

Parker, E.N.: 1963, Interplanetary Dynamical Processes, Interscience, New York. ADS.

Parker, E.N.: 1972, Topological dissipation and the small-scale fields in turbulent gases. Astrophys. J. 174, 499. DOI. ADS.

Parker, E.N.: 1978, Hydraulic concentration of magnetic fields in the solar photosphere. VI. Adiabatic cooling and concentration in downdrafts. Astrophys. J. 221, 368. DOI. ADS.

Parker, E.N.: 1979, Cosmical Magnetic Fields. Their Origin and Their Activity, Clarendon Press, Oxford. ADS.

Parker, E.N.: 1983, Magnetic neutral sheets in evolving fields - part two - formation of the solar corona. Astrophys. J. 264, 642. DOI. ADS.

Parnell, C.E., De Moortel, I.: 2012, A contemporary view of coronal heating. Phil. Trans. Roy. Soc. London Ser. A 370, 3217. DOI. ADS.

Peck, C.L., Rast, M.P.: 2015, Photometric trends in the visible solar continuum and their sensitivity to the center-to-limb profile. Astrophys. J. 808, 192. DOI. ADS.

Peck, C.L., Rast, M.P., Criscuoli, S., Rempel, M.: 2019, The solar photospheric continuum brightness as a function of mean magnetic flux density. I. The role of the magnetic structure size distribution. Astrophys. J. 870, 89. DOI. ADS.

Pereira, T.M.D.: 2019, The dynamic chromosphere: pushing the boundaries of observations and models. Adv. Space Res. 63, 1434. DOI. ADS.

Pereira, T.M.D., De Pontieu, B., Carlsson, M.: 2012, Quantifying spicules. Astrophys. J. 759, 18. DOI. ADS.

Pereira, T.M.D., De Pontieu, B., Carlsson, M., Hansteen, V., Tarbell, T.D., Lemen, J., Title, A., Boerner, P., Hurlburt, N., Wülser, J.P., Martínez-Sykora, J., Kleint, L., Golub, L., McKillop, S., Reeves, K.K., Saar, S., Testa, P., Tian, H., Jaeggli, S., Kankelborg, C.: 2014, An interface region imaging spectrograph first view on solar spicules. Astrophys. J. Lett. 792, L15. DOI. ADS.

Peter, H., Huang, Y.-M., Chitta, L.P., Young, P.R.: 2019, Plasmoid-mediated reconnection in solar UV bursts. Astron. Astrophys. 628, A8. DOI. ADS.

Petrie, G.: 2017, High-resolution vector magnetograms of the Sun's poles from Hinode: flux distributions and global coronal modeling. Solar Phys. 292, 13. DOI. ADS.

Petrie, G.J.D.: 2015, Solar magnetism in the polar regions. Liv. Rev. Solar Phys. 12, 5. DOI. ADS.

Petrie, G.J.D.: 2019, Abrupt changes in the photospheric magnetic field, Lorentz force, and magnetic shear during 15 X-class flares. Astrophys. J. Suppl. Ser. 240, 11. DOI. ADS. 
Pevtsov, A.A., Balasubramaniam, K.S.: 2003, Helicity patterns on the Sun. Adv. Space Res. 32, 1867. DOI. ADS.

Pevtsov, A.A., Berger, M.A., Nindos, A., Norton, A.A., van Driel-Gesztelyi, L.: 2014, Magnetic helicity, tilt, and twist. Space Sci. Rev. 186, 285. DOI. ADS.

Phillips, A.D., MacNeice, P.J., Antiochos, S.K.: 2005, The role of magnetic helicity in coronal mass ejections. Astrophys. J. Lett. 624, L129. DOI. ADS.

Pietarila, A., Hirzberger, J., Zakharov, V., Solanki, S.K.: 2009, Bright fibrils in Ca II K. Astron. Astrophys. 502, 647. DOI. ADS.

Plowman, J.E., Kankelborg, C.C., Longcope, D.W.: 2009, Coronal loop expansion properties explained using separators. Astrophys. J. 706, 108. DOI. ADS.

Poduval, B.: 2016, Controlling influence of magnetic field on solar wind outflow: an investigation using current sheet source surface model. Astrophys. J. Lett. 827, L6. DOI. ADS.

Poduval, B., Zhao, X.P.: 2014, Validating solar wind prediction using the current sheet source surface model. Astrophys. J. Lett. 782, L22. DOI. ADS.

Poduval, B., et al.: 2021, Astrophys. J. (in preparation).

Poedts, S.: 2002, MHD waves and heating of the solar corona. In: Sawaya-Lacoste, H. (ed.) SOLMAG 2002. Proc. Magnetic Coupling of the Solar Atmosphere Euroconference CS-505, ESA, Noordwijk, 273. ADS.

Polito, V., Testa, P., Allred, J., De Pontieu, B., Carlsson, M., Pereira, T.M.D., Gošić, M., Reale, F.: 2018, Investigating the response of loop plasma to nanoflare heating using RADYN simulations. Astrophys. $J$. 856, 178. DOI. ADS.

Pontin, D.I., Janvier, M., Tiwari, S.K., Galsgaard, K., Winebarger, A.R., Cirtain, J.W.: 2017, Observable signatures of energy release in braided coronal loops. Astrophys. J. 837, 108. DOI. ADS.

Popescu Braileanu, B., Lukin, V.S., Khomenko, E., de Vicente, Á.: 2019a, Two-fluid simulations of waves in the solar chromosphere. I. Numerical code verification. Astron. Astrophys. 627, A25. DOI. ADS.

Popescu Braileanu, B., Lukin, V.S., Khomenko, E., de Vicente, Á.: 2019b, Two-fluid simulations of waves in the solar chromosphere. II. Propagation and damping of fast magneto-acoustic waves and shocks. Astron. Astrophys. 630, A79. DOI. ADS.

Potter, A.E., Killen, R.M., Reardon, K.P., Bida, T.A.: 2013, Observation of neutral sodium above Mercury during the transit of November 8, 2006. Icarus 226, 172. DOI. ADS.

Pouquet, A., Patterson, G.S.: 1978, Numerical simulation of helical magnetohydrodynamic turbulence. $J$. Fluid Mech. 85, 305. DOI. ADS.

Pouquet, A., Frisch, U., Leorat, J.: 1976, Strong MHD helical turbulence and the nonlinear dynamo effect. J. Fluid Mech. 77, 321. DOI. ADS.

Priest, E.: 2014, Magnetohydrodynamics of the Sun, Cambridge Univ. Press, Cambridge. DOI. ADS.

Priest, E.R.: 2000, Solar Magneto-Hydrodynamics, Kluwer, Dordrecht.

Priest, E.R., Chitta, L.P., Syntelis, P.: 2018, A cancellation nanoflare model for solar chromospheric and coronal heating. Astrophys. J. Lett. 862, L24. DOI. ADS.

Qi, Y., Huang, Z., Xia, L., Li, B., Fu, H., Liu, W., Sun, M., Hou, Z.: 2019, On the relation between transition region network jets and coronal plumes. Solar Phys. 294, 92. DOI. ADS.

Qiu, J., Lee, J., Gary, D.E., Wang, H.: 2002, Motion of flare footpoint emission and inferred electric field in reconnecting current sheets. Astrophys. J. 565, 1335. DOI. ADS.

Rackham, B.V., Apai, D., Giampapa, M.S.: 2019, The transit light source effect. II. The impact of stellar heterogeneity on transmission spectra of planets orbiting broadly Sun-like stars. Astron. J. 157, 96. DOI. ADS.

Raouafi, N.E., Riley, P., Gibson, S., Fineschi, S., Solanki, S.K.: 2016, Diagnostics of coronal magnetic fields through the Hanle effect in UV and IR lines. Front. Astron. Space Sci. 3, 20. DOI. ADS.

Rast, M.P.: 1995, On the nature of "exploding" granules and granule fragmentation. Astrophys. J. 443, 863. DOI. ADS.

Rast, M.P.: 1998, Compressible plume dynamics and stability. J. Fluid Mech. 369, 125. DOI. ADS.

Rast, M.P.: 1999, The thermal starting plume as an acoustic source. Astrophys. J. 524, 462. DOI. ADS.

Rast, M.P., Bogdan, T.J.: 1998, On the asymmetry of solar acoustic line profiles. Astrophys. J. 496, 527. DOI. ADS.

Rast, M.P., Trampedach, R.: 2021, On the superadiabatic gradient in stellar convective envelopes. Astrophys. $J$. (in preparation).

Raymond, J.C., McCauley, P.I., Cranmer, S.R., Downs, C.: 2014, The solar corona as probed by Comet Lovejoy (C/2011 W3). Astrophys. J. 788, 152. DOI. ADS.

Raymond, J.C., Battams, K., Pesnell, W.D., Downs, C., Knight, M., Jia, Y.D., Wooden, D., Liu, W.: 2019 (private communications).

Reardon, K.P., Lepreti, F., Carbone, V., Vecchio, A.: 2008, Evidence of shock-driven turbulence in the solar chromosphere. Astrophys. J. Lett. 683, L207. DOI. ADS. 
Reep, J.W., Russell, A.J.B.: 2016, Alfvénic wave heating of the upper chromosphere in flares. Astrophys. J. Lett. 818, L20. DOI. ADS.

Reep, J.W., Antolin, P., Bradshaw, S.J.: 2020, Electron beams cannot directly produce coronal rain. Astrophys. J. 890, 100. DOI. ADS.

Reep, J.W., Russell, A.J.B., Tarr, L.A., Leake, J.E.: 2018, A hydrodynamic model of Alfvénic wave heating in a coronal loop and its chromospheric footpoints. Astrophys. J. 853, 101. DOI. ADS.

Reid, A., Mathioudakis, M., Doyle, J.G., Scullion, E., Nelson, C.J., Henriques, V., Ray, T.: 2016, Magnetic flux cancellation in Ellerman bombs. Astrophys. J. 823, 110. DOI. ADS.

Rempel, M.: 2011, Subsurface magnetic field and flow structure of simulated sunspots. Astrophys. J. 740, 15. DOI. ADS.

Rempel, M.: 2012a, Numerical models of sunspot formation and fine structure. Phil. Trans. Roy. Soc. London Ser. A 370, 3114. DOI. ADS.

Rempel, M.: 2012b, Numerical sunspot models: robustness of photospheric velocity and magnetic field structure. Astrophys. J. 750, 62. DOI. ADS.

Rempel, M.: 2014, Numerical simulations of quiet Sun magnetism: on the contribution from a small-scale dynamo. Astrophys. J. 789, 132. DOI. ADS.

Rempel, M.: 2018, Small-scale dynamo simulations: magnetic field amplification in exploding granules and the role of deep and shallow recirculation. Astrophys. J. 859, 161. DOI. ADS.

Rempel, M., Schlichenmaier, R.: 2011, Sunspot modeling: from simplified models to radiative MHD simulations. Liv. Rev. Solar Phys. 8, 3. DOI. ADS.

Rempel, M., Schüssler, M., Knölker, M.: 2009, Radiative magnetohydrodynamic simulation of sunspot structure. Astrophys. J. 691, 640. DOI. ADS.

Rezaei, R., Bello González, N., Schlichenmaier, R.: 2012, The formation of sunspot penumbra. Magnetic field properties. Astron. Astrophys. 537, A19. DOI. ADS.

Riley, P., Lionello, R., Mikić, Z., Linker, J., Clark, E., Lin, J., Ko, Y.-K.: 2007, "Bursty” reconnection following solar eruptions: MHD simulations and comparison with observations. Astrophys. J. 655, 591. DOI. ADS.

Riley, P., Lionello, R., Mikić, Z., Linker, J.: 2008, Using global simulations to relate the three-part structure of coronal mass ejections to in situ signatures. Astrophys. J. 672, 1221. DOI. ADS.

Riley, P., Linker, J.A., Mikic, Z., Caplan, R.M., Downs, C., Thumm, J.-L.: 2019, Can an unobserved concentration of magnetic flux above the poles of the Sun resolve the open flux problem? Astrophys. J. 884, 18. DOI. ADS.

Rimmele, T.R., Goode, P.R., Harold, E., Stebbins, R.T.: 1995, Dark lanes in granulation and the excitation of solar oscillations. Astrophys. J. Lett. 444, L119. DOI. ADS.

Rimmele, T.R., Warner, M., Keil, S.L., Goode, P.R., Knölker, M., Kuhn, J.R., Rosner, R.R., McMullin, J.P., Casini, R., Lin, H., Wöger, F., von der Lühe, O., Tritschler, A., Davey, A., de Wijn, A., Elmore, D.F., Fehlmann, A., Harrington, D.M., Jaeggli, S.A., Rast, M.P., Schad, T.A., Schmidt, W., Mathioudakis, M., Mickey, D.L., Anan, T., Beck, C., Marshall, H.K., Jeffers, P.F., Oschmann, J.M., Beard, A., Berst, D.C., Cowan, B.A., Craig, S.C., Cross, E., Cummings, B.K., Donnelly, C., de Vanssay, J.-B., Eigenbrot, A.D., Ferayorni, A., Foster, C., Galapon, C.A., Gedrites, C., Gonzales, K., Goodrich, B.D., Gregory, B.S., Guzman, S.S., Guzzo, S., Hegwer, S., Hubbard, R.P., Hubbard, J.R., Johansson, E.M., Johnson, L.C., Liang, C., Liang, M., McQuillen, I., Mayer, C., Newman, K., Onodera, B., Phelps, L., Puentes, M.M., Richards, C., Rimmele, L.M., Sekulic, P., Shimko, S.R., Simison, B.E., Smith, B., Starman, E., Sueoka, S.R., Summers, R.T., Szabo, A., Szabo, L., Wampler, S.B., Williams, T.R., White, C.: 2020, The Daniel K. Inouye Solar Telescope - observatory overview. Solar Phys. 295, 172. DOI. ADS.

Roberts, B.: 1981a, Wave propagation in a magnetically structured atmosphere - part one - surface waves at a magnetic interface. Solar Phys. 69, 27. DOI. ADS.

Roberts, B.: 1981b, Wave propagation in a magnetically structured atmosphere - part two - waves in a magnetic slab. Solar Phys. 69, 39. DOI. ADS.

Roberts, B.: 1983, Wave propagation in intense flux tubes. Solar Phys. 87, 77. DOI. ADS.

Roberts, B.: 2000, Waves and oscillations in the corona - (invited review). Solar Phys. 193, 139. DOI. ADS.

Roberts, B.: 2006, Slow magnetohydrodynamic waves in the solar atmosphere. Phil. Trans. Roy. Soc. London Ser. A 364, 447. DOI. ADS.

Robustini, C., Leenaarts, J., de la Cruz Rodriguez, J., Rouppe van der Voort, L.: 2016, Fan-shaped jets above the light bridge of a sunspot driven by reconnection. Astron. Astrophys. 590, A57. DOI. ADS.

Röhrbein, D., Cameron, R., Schüssler, M.: 2011, Is there a non-monotonic relation between photospheric brightness and magnetic field strength in solar plage regions? Astron. Astrophys. 532, A140. DOI. ADS.

Roth, M., Franz, M., Bello González, N., Martínez Pillet, V., Bonet, J.A., Gandorfer, A., Barthol, P., Solanki, S.K., Berkefeld, T., Schmidt, W., del Toro Iniesta, J.C., Domingo, V., Knölker, M.: 2010, Surface waves in solar granulation observed with SUNRISE. Astrophys. J. Lett. 723, L175. DOI. ADS. 
Rouillard, A.P., Kouloumvakos, A., Vourlidas, A., Kasper, J., Bale, S., Raouafi, N.-E., Lavraud, B., Howard, R.A., Stenborg, G., Stevens, M., Poirier, N., Davies, J.A., Hess, P., Higginson, A.K., Lavarra, M., Viall, N.M., Korreck, K., Pinto, R.F., Griton, L., Réville, V., Louarn, P., Wu, Y., Dalmasse, K., Génot, V., Case, A.W., Whittlesey, P., Larson, D., Halekas, J.S., Livi, R., Goetz, K., Harvey, P.R., MacDowall, R.J., Malaspina, D., Pulupa, M., Bonnell, J., de Witt, T.D., Penou, E.: 2020, Relating streamer flows to density and magnetic structures at the Parker solar probe. Astrophys. J. Suppl. Ser. 246, 37. DOI. ADS.

Rouppe van der Voort, L., de la Cruz Rodríguez, J.: 2013, Short dynamic fibrils in sunspot chromospheres. Astrophys. J. 776, 56. DOI. ADS.

Rouppe van der Voort, L.H.M., Rutten, R.J., Vissers, G.J.M.: 2016, Reconnection brightenings in the quiet solar photosphere. Astron. Astrophys. 592, A100. DOI. ADS.

Rouppe van der Voort, L., De Pontieu, B., Scharmer, G.B., de la Cruz Rodríguez, J., Martínez-Sykora, J., Nóbrega-Siverio, D., Guo, L.J., Jafarzadeh, S., Pereira, T.M.D., Hansteen, V.H., Carlsson, M., Vissers, G.: 2017, Intermittent reconnection and plasmoids in UV bursts in the low solar atmosphere. Astrophys. J. Lett. 851, L6. DOI. ADS.

Roy, J.-R., Leparskas, H.: 1973, Some statistical properties of Ellerman bombs. Solar Phys. 30, 449. DOI. ADS.

Ruderman, M.S., Erdélyi, R.: 2009, Transverse oscillations of coronal loops. Space Sci. Rev. 149, 199. DOI. ADS.

Russell, A.J.B., Fletcher, L.: 2013, Propagation of Alfvénic waves from corona to chromosphere and consequences for solar flares. Astrophys. J. 765, 81. DOI. ADS.

Russell, A.J.B., Simões, P.J.A., Fletcher, L.: 2015, A unified view of coronal loop contraction and oscillation in flares. Astron. Astrophys. 581, A8. DOI. ADS.

Rutten, R.J., Vissers, G.J.M., Rouppe van der Voort, L.H.M., Sütterlin, P., Vitas, N.: 2013, Ellerman bombs: fallacies, fads, usage. In: Cally, P., Erdélyi, R., Norton, A. (eds.) Eclipse on the Coral Sea: Cycle 24 Ascending (GONG 2012, LWS/SDO-5, and SOHO 27), J. Phys. CS-440. DOI. ADS.

Salabert, D., García, R.A., Turck-Chièze, S.: 2015, Seismic sensitivity to sub-surface solar activity from 18 yr of GOLF/SoHO observations. Astron. Astrophys. 578, A137. DOI. ADS.

Samanta, T., Tian, H., Prasad Choudhary, D.: 2018, Statistical investigation of supersonic downflows in the transition region above sunspots. Astrophys. J. 859, 158. DOI. ADS.

Samanta, T., Tian, H., Banerjee, D., Schanche, N.: 2017, Dynamics of subarcsecond bright dots in the transition region above sunspots and their relation to penumbral micro-jets. Astrophys. J. Lett. 835, L19. DOI. ADS.

Samanta, T., Tian, H., Yurchyshyn, V., Peter, H., Cao, W., Sterling, A., Erdélyi, R., Ahn, K., Feng, S., Utz, D., Banerjee, D., Chen, Y.: 2019, Generation of solar spicules and subsequent atmospheric heating. Science 366, 890. DOI. ADS.

Santamaria, I.C., Khomenko, E., Collados, M., de Vicente, A.: 2016, Simulated interaction of magnetohydrodynamic shock waves with a complex network-like region. Astron. Astrophys. 590, L3. DOI. ADS.

Sasso, C., Lagg, A., Solanki, S.K.: 2014, Magnetic structure of an activated filament in a flaring active region. Astron. Astrophys. 561, A98. DOI. ADS.

Savage, S.L., McKenzie, D.E., Reeves, K.K., Forbes, T.G., Longcope, D.W.: 2010, Reconnection outflows and current sheet observed with Hinode/XRT in the 2008 April 9 "Cartwheel CME" flare. Astrophys. J. 722, 329. DOI. ADS.

Scalo, J., Kaltenegger, L., Segura, A.G., Fridlund, M., Ribas, I., Kulikov, Y.N., Grenfell, J.L., Rauer, H., Odert, P., Leitzinger, M., Selsis, F., Khodachenko, M.L., Eiroa, C., Kasting, J., Lammer, H.: 2007, M stars as targets for terrestrial exoplanet searches and biosignature detection. Astrobiology 7, 85. DOI. ADS.

Schad, T.A.: 2018, Neutral helium triplet spectroscopy of quiescent coronal rain with sensitivity estimates for spectropolarimetric magnetic field diagnostics. Astrophys. J. 865, 31. DOI. ADS.

Schad, T.A., Penn, M.J., Lin, H.: 2013, He I vector magnetometry of field-aligned superpenumbral fibrils. Astrophys. J. 768, 111. DOI. ADS.

Scharmer, G.B., Bjelksjo, K., Korhonen, T.K., Lindberg, B., Petterson, B.: 2003, The 1-meter Swedish solar telescope. In: Keil, S.L., Avakyan, S.V. (eds.) Innovative Telescopes and Instrumentation for Solar Astrophysics, Proc. SPIE CS-4853, 341. DOI. ADS.

Scharmer, G.B., Narayan, G., Hillberg, T., de la Cruz Rodriguez, J., Löfdahl, M.G., Kiselman, D., Sütterlin, P., van Noort, M., Lagg, A.: 2008, CRISP spectropolarimetric imaging of penumbral fine structure. Astrophys. J. Lett. 689, L69. DOI. ADS.

Scheeler, M.W., van Rees, W.M., Kedia, H., Kleckner, D., Irvine, W.T.M.: 2017, Complete measurement of helicity and its dynamics in vortex tubes. Science 357, 487. DOI. ADS.

Schleicher, H., Wiedemann, G., Wöhl, H., Berkefeld, T., Soltau, D.: 2004, Detection of neutral sodium above Mercury during the transit on 2003 May 7. Astron. Astrophys. 425, 1119. DOI. ADS. 
Schlichenmaier, R., González, N.B., Rezaei, R.: 2011, The formation of a penumbra as observed with the German VTT and SoHO/MDI. In: Prasad Choudhary, D., Strassmeier, K.G. (eds.) Physics of Sun and Star Spots, IAU Symp. 273, Cambridge Univ. Press, Cambridge, 134. DOI. ADS.

Schmelz, J.T., Winebarger, A.R.: 2015, What can observations tell us about coronal heating? Phil. Trans. Roy. Soc. London Ser. A 373, 20140257. DOI. ADS.

Schmidt, C.A., Leblanc, F., Reardon, K., Killen, R.M., Gary, D.E., Ahn, K.: 2018, Absorption spectroscopy of Mercury's exosphere during the 2016 solar transit. In: Mercury: Current and Future Science of the Innermost Planets, Lunar and Planetary Institute, Houston, Abstract \#6022.

Schnerr, R.S., Spruit, H.C.: 2011, The brightness of magnetic field concentrations in the quiet Sun. Astron. Astrophys. 532, A136. DOI. ADS.

Schrijver, C.J.: 2001, Catastrophic cooling and high-speed downflow in quiescent solar coronal loops observed with TRACE. Solar Phys. 198, 325. DOI. ADS.

Schrijver, C.J.: 2007, Braiding-induced interchange reconnection of the magnetic field and the width of solar coronal loops. Astrophys. J. Lett. 662, L119. DOI. ADS.

Schrijver, C.J.: 2009, Driving major solar flares and eruptions: a review. Adv. Space Res. 43, 739. DOI. ADS.

Schrijver, C.J., Title, A.M.: 2003, The magnetic connection between the solar photosphere and the corona. Astrophys. J. Lett. 597, L165. DOI. ADS.

Schrijver, C.J., Brown, J.C., Battams, K., Saint-Hilaire, P., Liu, W., Hudson, H., Pesnell, W.D.: 2012, Destruction of Sun-grazing comet C/2011 N3 (SOHO) within the low solar corona. Science 335, 324. DOI. ADS.

Schuck, P.W., Chen, J., Schwartz, I.B., Yurchyshyn, V.: 2004, On the temporal relationship between H $\alpha$ filament eruptions and soft X-ray emissions. Astrophys. J. Lett. 610, L133. DOI. ADS.

Schüssler, M., Vögler, A.: 2006, Magnetoconvection in a sunspot umbra. Astrophys. J. Lett. 641, L73. DOI. ADS.

Schwarzschild, M.: 1948, On noise arising from the solar granulation. Astrophys. J. 107, 1. DOI. ADS.

Scullion, E., Rouppe van der Voort, L., Wedemeyer, S., Antolin, P.: 2014, Unresolved fine-scale structure in solar coronal loop-tops. Astrophys. J. 797, 36. DOI. ADS.

Seaton, D.B., Bartz, A.E., Darnel, J.M.: 2017, Observations of the formation, development, and structure of a current sheet in an eruptive solar flare. Astrophys. J. 835, 139. DOI. ADS.

Seo, B., Wongwaitayakornkul, P., Haw, M.A., Marshall, R.S., Li, H., Bellan, P.M.: 2020, Determination of a macro- to micro-scale progression leading to a magnetized plasma disruption. Phys. Plasmas 27, 022109. DOI. ADS.

Severino, G., Magrì, M., Oliviero, M., Straus, T., Jefferies, S.M.: 2001, The solar intensity-velocity cross spectrum: a powerful diagnostic for helioseismology. Astrophys. J. 561, 444. DOI. ADS.

Sharykin, I.N., Kosovichev, A.G.: 2014, Fine structure of flare ribbons and evolution of electric currents. Astrophys. J. Lett. 788, L18. DOI. ADS.

Shelyag, S., Keys, P., Mathioudakis, M., Keenan, F.P.: 2011, Vorticity in the solar photosphere. Astron. Astrophys. 526, A5. DOI. ADS.

Sheminova, V.A.: 2019, Turbulence and rotation in solar-type stars. Kinemat. Phys. Celest. Bodies 35, 129. DOI. ADS.

Shetye, J., Shelyag, S., Reid, A.L., Scullion, E., Doyle, J.G., Arber, T.D.: 2018, Signatures of quiet Sun reconnection events in $\mathrm{Ca}$ II, $\mathrm{H} \alpha$, and Fe I. Mon. Not. Roy. Astron. Soc. 479, 3274. DOI. ADS.

Shetye, J., Verwichte, E., Stangalini, M., Judge, P.G., Doyle, J.G., Arber, T., Scullion, E., Wedemeyer, S.: 2019, Multiwavelength high-resolution observations of chromospheric swirls in the quiet Sun. Astrophys. J. 881, 83. DOI. ADS.

Shibata, K., Magara, T.: 2011, Solar flares: magnetohydrodynamic processes. Liv. Rev. Solar Phys. 8, 6. DOI. ADS.

Shibata, K., Nakamura, T., Matsumoto, T., Otsuji, K., Okamoto, T.J., Nishizuka, N., Kawate, T., Watanabe, H., Nagata, S., UeNo, S., Kitai, R., Nozawa, S., Tsuneta, S., Suematsu, Y., Ichimoto, K., Shimizu, T., Katsukawa, Y., Tarbell, T.D., Berger, T.E., Lites, B.W., Shine, R.A., Title, A.M.: 2007, Chromospheric anemone jets as evidence of ubiquitous reconnection. Science 318, 1591. DOI. ADS.

Shimizu, T.: 2015, 3D magnetic field configuration of small-scale reconnection events in the solar plasma atmosphere. Phys. Plasmas, 22(10), 101207. DOI. ADS.

Shimizu, T., Ichimoto, K., Suematsu, Y.: 2012, Precursor of sunspot penumbral formation discovered with Hinode solar optical telescope observations. Astrophys. J. Lett. 747, L18. DOI. ADS.

Shimizu, T., Katsukawa, Y., Kubo, M., Lites, B.W., Ichimoto, K., Suematsu, Y., Tsuneta, S., Nagata, S., Shine, R.A., Tarbell, T.D.: 2009, Hinode observation of the magnetic fields in a sunspot light bridge accompanied by long-lasting chromospheric plasma ejections. Astrophys. J. Lett. 696, L66. DOI. ADS.

Singh, K.A.P., Isobe, H., Nishizuka, N., Nishida, K., Shibata, K.: 2012, Multiple plasma ejections and intermittent nature of magnetic reconnection in solar chromospheric anemone jets. Astrophys. J. 759, 33. DOI. ADS. 
Siu-Tapia, A., Lagg, A., Solanki, S.K., van Noort, M., Jurčák, J.: 2017, Normal and counter Evershed flows in the photospheric penumbra of a sunspot. SPINOR 2D inversions of Hinode-SOT/SP observations. Astron. Astrophys. 607, A36. DOI. ADS.

Skartlien, R., Rast, M.P.: 2000, p-Mode intensity-velocity phase differences and convective sources. Astrophys. J. 535, 464. DOI. ADS.

Skogsrud, H., Rouppe van der Voort, L., De Pontieu, B., Pereira, T.M.D.: 2015, On the temporal evolution of spicules observed with IRIS, SDO, and Hinode. Astrophys. J. 806(2), 170. DOI. ADS.

Slavin, J.A., Acuña, M.H., Anderson, B.J., Baker, D.N., Benna, M., Boardsen, S.A., Gloeckler, G., Gold, R.E., Ho, G.C., Korth, H., Krimigis, S.M., McNutt, R.L., Raines, J.M., Sarantos, M., Schriver, D., Solomon, S.C., Trávníček, P., Zurbuchen, T.H.: 2009, MESSENGER observations of magnetic reconnection in Mercury's magnetosphere. Science 324, 606. DOI. ADS.

Slavin, J.A., Anderson, B.J., Baker, D.N., Benna, M., Boardsen, S.A., Gloeckler, G., Gold, R.E., Ho, G.C., Korth, H., Krimigis, S.M., McNutt, R.L., Nittler, L.R., Raines, J.M., Sarantos, M., Schriver, D., Solomon, S.C., Starr, R.D., Trávníček, P.M., Zurbuchen, T.H.: 2010, MESSENGER observations of extreme loading and unloading of Mercury's magnetic tail. Science 329, 665. DOI. ADS.

Smith, M.D.: 1989, The stability of radiative shocks. Mon. Not. Roy. Astron. Soc. 238, 235. DOI. ADS.

Smitha, H.N., Chitta, L.P., Wiegelmann, T., Solanki, S.K.: 2018, Observations of solar chromospheric heating at sub-arcsec spatial resolution. Astron. Astrophys. 617, A128. DOI. ADS.

Snow, B., Botha, G.J.J., Scullion, E., McLaughlin, J.A., Young, P.R., Jaeggli, S.A.: 2018, Predictions of DKIST/DL-NIRSP observations for an off-limb kink-unstable coronal loop. Astrophys. J. 863, 172. DOI. ADS.

Socas-Navarro, H., Martínez Pillet, V., Elmore, D., Pietarila, A., Lites, B.W., Manso Sainz, R.: 2006, Spectropolarimetric observations and non-Lte modeling of Ellerman bombs. Solar Phys. 235, 75. DOI. ADS.

Socas-Navarro, H., McIntosh, S.W., Centeno, R., de Wijn, A.G., Lites, B.W.: 2009, Direct imaging of fine structure in the chromosphere of a sunspot umbra. Astrophys. J. 696, 1683. DOI. ADS.

Solanki, S.K., Barthol, P., Danilovic, S., Feller, A., Gandorfer, A., Hirzberger, J., Riethmüller, T.L., Schüssler, M., Bonet, J.A., Martínez Pillet, V., del Toro Iniesta, J.C., Domingo, V., Palacios, J., Knölker, M., Bello González, N., Berkefeld, T., Franz, M., Schmidt, W., Title, A.M.: 2010, SUNRISE: instrument, mission, data, and first results. Astrophys. J. Lett. 723, L127. DOI. ADS.

Song, H.Q., Kong, X.L., Chen, Y., Li, B., Li, G., Feng, S.W., Xia, L.D.: 2012, A statistical study on the morphology of rays and dynamics of blobs in the wake of coronal mass ejections. Solar Phys. 276, 261. DOI. ADS.

Spruit, H.C.: 1979, Convective collapse of flux tubes. Solar Phys. 61, 363. DOI. ADS.

Spruit, H.C., Bogdan, T.J.: 1992, The conversion of p-modes to slow modes and the absorption of acoustic waves by sunspots. Astrophys. J. Lett. 391, L109. DOI. ADS.

Spruit, H.C., Zweibel, E.G.: 1979, Convective instability of thin flux tubes. Solar Phys. 62, 15. DOI. ADS.

Squire, J., Chandran, B.D.G., Meyrand, R.: 2020, In-situ switchback formation in the expanding solar wind. Astrophys. J. Lett. 891, L2. DOI. ADS.

Stein, R.F., Leibacher, J.: 1974, Waves in the solar atmosphere. Annu. Rev. Astron. Astrophys. 12, 407. DOI. ADS.

Stein, R.F., Nordlund, Å.: 1991, Convection and its influence on oscillations. In: Gough, D., Toomre, J. (eds.) Challenges to Theories of the Structure of Moderate-Mass Stars, Lecture Notes in Physics 388, Springer, Berlin, 195. DOI. ADS.

Stein, R.F., Nordlund, ̊.: 1998, Simulations of solar granulation. I. General properties. Astrophys. J. 499, 914. DOI. ADS.

Stein, R.F., Nordlund, Å.: 2000, Realistic solar convection simulations. Solar Phys. 192, 91. DOI. ADS.

Stein, R.F., Bercik, D., Nordlund, Å.: 2003, Solar surface magneto-convection. In: Pevtsov, A.A., Uitenbroek, H. (eds.) Current Theoretical Models and Future High Resolution Solar Observations: Preparing for ATST CS-286, Astron. Soc. Pacific, San Francisco, 121. ADS.

Stenflo, J.O.: 1975, A model of the supergranulation network and of active region plages. Solar Phys. $42,79$. DOI. ADS.

Sterling, A.C.: 2000, Solar spicules: a review of recent models and targets for future observations - (invited review). Solar Phys. 196, 79. DOI. ADS.

Sterling, A.C., Moore, R.L.: 2016, A microfilament-eruption mechanism for solar spicules. Astrophys. J. Lett. 828, L9. DOI. ADS.

Sterling, A.C., Moore, R.L., Falconer, D.A., Adams, M.: 2015, Small-scale filament eruptions as the driver of X-ray jets in solar coronal holes. Nature 523, 437. DOI. ADS.

Straus, T., Severino, G., Deubner, F.-L., Fleck, B., Jefferies, S.M., Tarbell, T.: 1999, Observational constraints on models of the solar background spectrum. Astrophys. J. 516, 939. DOI. ADS.

Straus, T., Fleck, B., Jefferies, S.M., Cauzzi, G., McIntosh, S.W., Reardon, K., Severino, G., Steffen, M.: 2008, The energy flux of internal gravity waves in the lower solar atmosphere. Astrophys. J. Lett. 681, L125. DOI. ADS 
Sturrock, P.A.: 1999, Chromospheric magnetic reconnection and its possible relationship to coronal heating. Astrophys. J. 521, 451. DOI. ADS.

Sturrock, Z., Hood, A.W., Archontis, V., McNeill, C.M.: 2015, Sunspot rotation. I. A consequence of flux emergence. Astron. Astrophys. 582, A76. DOI. ADS.

Su, Y., Liu, R., Li, S., Cao, W., Ahn, K., Ji, H.: 2018, High-resolution observations of flares in an arch filament system. Astrophys. J. 855, 77. DOI. ADS.

Sudol, J.J., Harvey, J.W.: 2005, Longitudinal magnetic field changes accompanying solar flares. Astrophys. J. 635, 647. DOI. ADS.

Sullivan, P.P., Patton, E.G.: 2011, The effect of mesh resolution on convective boundary layer statistics and structures generated by large-eddy simulation. J. Atmos. Sci. 68, 2395. DOI. ADS.

Sun, X., Hoeksema, J.T., Liu, Y., Wiegelmann, T., Hayashi, K., Chen, Q., Thalmann, J.: 2012, Evolution of magnetic field and energy in a major eruptive active region based on SDO/HMI observation. Astrophys. J. 748, 77. DOI. ADS.

Sun, X., Hoeksema, J.T., Liu, Y., Kazachenko, M., Chen, R.: 2017, Investigating the magnetic imprints of major solar eruptions with SDO/HMI high-cadence vector magnetograms. Astrophys. J. 839, 67. DOI. ADS.

Takahata, Y., Yanai, R., Inomoto, M.: 2019, Experimental study of magnetic reconnection in partially ionized plasmas using rotating magnetic field. J. Plasma Fusion Res. 14, 3401054. DOI. ADS.

Takasao, S., Asai, A., Isobe, H., Shibata, K.: 2012, Simultaneous observation of reconnection inflow and outflow associated with the 2010 August 18 solar flare. Astrophys. J. Lett. 745, L6. DOI. ADS.

Takeda, Y., UeNo, S.: 2017, Does the radial-tangential macroturbulence model adequately describe the spectral line broadening of solar-type stars? Publ. Astron. Soc. Japan 69, 46. DOI. ADS.

Tappin, S.J.: 1991, Do all solar flares have X-ray precursors? Astron. Astrophys. Suppl. Ser. 87, 277. ADS.

Taylor, J.B.: 1974, Relaxation of toroidal plasma and generation of reverse magnetic fields. Phys. Rev. Lett. 33, 1139. DOI. ADS.

Tenerani, A., Velli, M., Matteini, L., Réville, V., Shi, C., Bale, S.D., Kasper, J.C., Bonnell, J.W., Case, A.W., de Wit, T.D., Goetz, K., Harvey, P.R., Klein, K.G., Korreck, K., Larson, D., Livi, R., MacDowall, R.J., Malaspina, D.M., Pulupa, M., Stevens, M., Whittlesey, P.: 2020, Magnetic field kinks and folds in the solar wind. Astrophys. J. Suppl. Ser. 246, 32. DOI. ADS.

Testa, P., De Pontieu, B., Allred, J., Carlsson, M., Reale, F., Daw, A., Hansteen, V., Martinez-Sykora, J., Liu, W., DeLuca, E.E., Golub, L., McKillop, S., Reeves, K., Saar, S., Tian, H., Lemen, J., Title, A., Boerner, P., Hurlburt, N., Tarbell, T.D., Wuelser, J.P., Kleint, L., Kankelborg, C., Jaeggli, S.: 2014, Evidence of nonthermal particles in coronal loops heated impulsively by nanoflares. Science 346, 1255724. DOI. ADS.

Thurgood, J.O., Morton, R.J., McLaughlin, J.A.: 2014, First direct measurements of transverse waves in solar polar plumes using SDO/AIA. Astrophys. J. Lett. 790, L2. DOI. ADS.

Tian, H., Tu, C.-Y., Marsch, E., He, J.-S., Zhou, G.-Q.: 2008, Signature of mass supply to quiet coronal loops. Astron. Astrophys. 478, 915. DOI. ADS.

Tian, H., Kleint, L., Peter, H., Weber, M., Testa, P., DeLuca, E., Golub, L., Schanche, N.: 2014, Observations of subarcsecond bright dots in the transition region above sunspots with the Interface Region Imaging Spectrograph. Astrophys. J. Lett. 790, L29. DOI. ADS.

Tian, H., Yurchyshyn, V., Peter, H., Solanki, S.K., Young, P.R., Ni, L., Cao, W., Ji, K., Zhu, Y., Zhang, J., Samanta, T., Song, Y., He, J., Wang, L., Chen, Y.: 2018, Frequently occurring reconnection jets from sunspot light bridges. Astrophys. J. 854, 92. DOI. ADS.

Tian, L., Alexander, D.: 2008, On the origin of magnetic helicity in the solar corona. Astrophys. J. 673, 532. DOI. ADS.

Tiwari, S.K., van Noort, M., Lagg, A., Solanki, S.K.: 2013, Structure of sunspot penumbral filaments: a remarkable uniformity of properties. Astron. Astrophys. 557, A25. DOI. ADS.

Tiwari, S.K., Moore, R.L., Winebarger, A.R., Alpert, S.E.: 2016, Transition-region/coronal signatures and magnetic setting of sunspot penumbral jets: Hinode (SOT/FG), Hi-C, and SDO/AIA observations. Astrophys. J. 816, 92. DOI. ADS.

Tiwari, S.K., Moore, R.L., De Pontieu, B., Tarbell, T.D., Panesar, N.K., Winebarger, A.R., Sterling, A.C.: 2018, Evidence of twisting and mixed-polarity solar photospheric magnetic field in large penumbral jets: IRIS and Hinode observations. Astrophys. J. 869, 147. DOI. ADS.

Tomczyk, S., McIntosh, S.W.: 2009, Time-distance seismology of the solar corona with CoMP. Astrophys. J. 697, 1384. DOI. ADS.

Tomczyk, S., McIntosh, S.W., Keil, S.L., Judge, P.G., Schad, T., Seeley, D.H., Edmondson, J.: 2007, Alfvén waves in the solar corona. Science 317, 1192. DOI. ADS.

Topka, K.P., Tarbell, T.D., Title, A.M.: 1997, Properties of the smallest solar magnetic elements. II. Observations versus hot wall models of faculae. Astrophys. J. 484, 479. DOI. ADS. 
Torbert, R.B., Burch, J.L., Phan, T.D., Hesse, M., Argall, M.R., Shuster, J., Ergun, R.E., Alm, L., Nakamura, R., Genestreti, K.J., Gershman, D.J., Paterson, W.R., Turner, D.L., Cohen, I., Giles, B.L., Pollock, C.J., Wang, S., Chen, L.-J., Stawarz, J.E., Eastwood, J.P., Hwang, K.J., Farrugia, C., Dors, I., Vaith, H., Mouikis, C., Ardakani, A., Mauk, B.H., Fuselier, S.A., Russell, C.T., Strangeway, R.J., Moore, T.E., Drake, J.F., Shay, M.A., Khotyaintsev, Y.V., Lindqvist, P.-A., Baumjohann, W., Wilder, F.D., Ahmadi, N., Dorelli, J.C., Avanov, L.A., Oka, M., Baker, D.N., Fennell, J.F., Blake, J.B., Jaynes, A.N., Le Contel, O., Petrinec, S.M., Lavraud, B., Saito, Y.: 2018, Electron-scale dynamics of the diffusion region during symmetric magnetic reconnection in space. Science 362, 1391. DOI. ADS.

Toriumi, S., Wang, H.: 2019, Flare-productive active regions. Liv. Rev. Solar Phys. 16, 3. DOI. ADS.

Toriumi, S., Katsukawa, Y., Cheung, M.C.M.: 2015, Light bridge in a developing active region. I. Observation of light bridge and its dynamic activity phenomena. Astrophys. J. 811, 137. DOI. ADS.

Toriumi, S., Katsukawa, Y., Cheung, M.C.M.: 2017, Various local heating events in the earliest phase of flux emergence. Astrophys. J. 836, 63. DOI. ADS.

Török, T., Leake, J.E., Titov, V.S., Archontis, V., Mikić, Z., Linton, M.G., Dalmasse, K., Aulanier, G., Kliem, B.: 2014, Distribution of electric currents in solar active regions. Astrophys. J. Lett. 782, L10. DOI. ADS.

Tritschler, A., et al.: 2021, The DKIST Operations Plan. Solar Phys. 296 (in preparation).

Trujillo Bueno, J., Shchukina, N., Asensio Ramos, A.: 2004, A substantial amount of hidden magnetic energy in the quiet Sun. Nature 430, 326. DOI. ADS.

Tsuneta, S., Ichimoto, K., Katsukawa, Y., Lites, B.W., Matsuzaki, K., Nagata, S., Orozco Suárez, D., Shimizu, T., Shimojo, M., Shine, R.A., Suematsu, Y., Suzuki, T.K., Tarbell, T.D., Title, A.M.: 2008, The magnetic landscape of the Sun's polar region. Astrophys. J. 688, 1374. DOI. ADS.

Tziotziou, K., Tsiropoula, G., Kontogiannis, I., Scullion, E., Doyle, J.G.: 2018, A persistent quiet-Sun smallscale tornado. I. Characteristics and dynamics. Astron. Astrophys. 618, A51. DOI. ADS.

Uitenbroek, H., Criscuoli, S.: 2011, Why one-dimensional models fail in the diagnosis of average spectra from inhomogeneous stellar atmospheres. Astrophys. J. 736, 69. DOI. ADS.

Ulmschneider, P., Zähringer, K., Musielak, Z.E.: 1991, Propagation of nonlinear longitudinal-transverse waves along magnetic flux tubes in the solar atmosphere. I - adiabatic waves. Astron. Astrophys. 241, 625. ADS.

Ulrich, R.K.: 1970, The five-minute oscillations on the solar surface. Astrophys. J. 162, 993. DOI. ADS.

van Ballegooijen, A.A.: 1999, Photospheric motions as a source of twist in coronal magnetic fields. In: Brown, M.R., Canfield, R.C., Pevtsov, A.A. (eds.) Magnetic Helicity in Space and Laboratory Plasmas, Geophys. Mono. Ser. 111, Am. Geophys. Union, Washington DC, 213. DOI. ADS.

van Ballegooijen, A.A., Martens, P.C.H.: 1989, Formation and eruption of solar prominences. Astrophys. J. 343, 971. DOI. ADS.

van Ballegooijen, A.A., Asgari-Targhi, M., Cranmer, S.R., DeLuca, E.E.: 2011, Heating of the solar chromosphere and corona by Alfvén wave turbulence. Astrophys. J. 736, 3. DOI. ADS.

van der Holst, B., Sokolov, I.V., Meng, X., Jin, M., Manchester, W.B. IV, Tóth, G., Gombosi, T.I.: 2014, Alfvén Wave Solar Model (AWSoM): coronal heating. Astrophys. J. 782, 81. DOI. ADS.

van der Holst, B., Manchester, W.B. IV, Klein, K.G., Kasper, J.C.: 2019, Predictions for the first Parker solar probe encounter. Astrophys. J. Lett. 872, L18. DOI. ADS.

Van Doorsselaere, T., Nakariakov, V.M., Verwichte, E.: 2008, Detection of waves in the solar corona: kink or Alfvén? Astrophys. J. Lett. 676, L73. DOI. ADS.

van Driel-Gesztelyi, L., Démoulin, P., Mandrini, C.H.: 2003, Observations of magnetic helicity. Adv. Space Res. 32, 1855. DOI. ADS.

van Heerwaarden, C.C., Mellado, J.P., De Lozar, A.: 2014, Scaling laws for the heterogeneously heated free convective boundary layer. J. Atmos. Sci. 71, 3975. DOI. ADS.

van Noort, M., Lagg, A., Tiwari, S.K., Solanki, S.K.: 2013, Peripheral downflows in sunspot penumbrae. Astron. Astrophys. 557, A24. DOI. ADS.

Vecchio, A., Cauzzi, G., Reardon, K.P.: 2009, The solar chromosphere at high resolution with IBIS. II. Acoustic shocks in the quiet internetwork and the role of magnetic fields. Astron. Astrophys. 494, 269. DOI. ADS.

Vecchio, A., Cauzzi, G., Reardon, K.P., Janssen, K., Rimmele, T.: 2007, Solar atmospheric oscillations and the chromospheric magnetic topology. Astron. Astrophys. 461, L1. DOI. ADS.

Velli, M., Pucci, F., Rappazzo, F., Tenerani, A.: 2015, Models of coronal heating, turbulence and fast reconnection. Phil. Trans. Roy. Soc. London Ser. A 373, 20140262. DOI. ADS.

Velli, M., Harra, L.K., Vourlidas, A., Schwadron, N., Panasenco, O., Liewer, P.C., Müller, D., Zouganelis, I., St. Cyr, O.C., Gilbert, H., Nieves-Chinchilla, T., Auchère, F., Berghmans, D., Fludra, A., Horbury, T.S., Howard, R.A., Krucker, S., Maksimovic, M., Owen, C.J., Rodríguez-Pacheco, J., Romoli, M., Solanki, S.K., Wimmer-Schweingruber, R.F., Bale, S., Kasper, J., McComas, D.J., Raouafi, N., Martinez-Pillet, 
V., Walsh, A.P., De Groof, A., Williams, D.: 2020, Understanding the origins of the heliosphere: integrating observations and measurements from Parker Solar Probe, Solar Orbiter, and other space- and ground-based observatories. Astron. Astrophys. 642, A4. DOI. ADS.

Venkatakrishnan, P.: 1986, Inhibition of convective collapse of solar magnetic flux tubes by radiative diffusion. Nature 322, 156. DOI. ADS.

Venzmer, M.S., Bothmer, V.: 2018, Solar-wind predictions for the Parker Solar Probe orbit. Near-Sun extrapolations derived from an empirical solar-wind model based on Helios and OMNI observations. Astron. Astrophys. 611, A36. DOI. ADS.

Vervack, R.J., Killen, R.M., McClintock, W.E., Merkel, A.W., Burger, M.H., Cassidy, T.A., Sarantos, M.: 2016, New discoveries from MESSENGER and insights into Mercury's exosphere. Geophys. Res. Lett. 43, 11,545. DOI. ADS.

Vigeesh, G., Jackiewicz, J., Steiner, O.: 2017, Internal gravity waves in the magnetized solar atmosphere. I. Magnetic field effects. Astrophys. J. 835, 148. DOI. ADS.

Vigeesh, G., Roth, M., Steiner, O., Jackiewicz, J.: 2019, Internal gravity waves in the magnetized solar atmosphere. II. Energy transport. Astrophys. J. 872, 166. DOI. ADS.

Vissers, G.J.M., Rouppe van der Voort, L.H.M., Carlsson, M.: 2015, Evidence for a transition region response to penumbral microjets in sunspots. Astrophys. J. Lett. 811, L33. DOI. ADS.

Vissers, G.J.M., Rouppe van der Voort, L.H.M., Rutten, R.J.: 2013, Ellerman bombs at high resolution. II. Triggering, visibility, and effect on upper atmosphere. Astrophys. J. 774, 32. DOI. ADS.

Vissers, G.J.M., Rouppe van der Voort, L.H.M., Rutten, R.J., Carlsson, M., De Pontieu, B.: 2015, Ellerman bombs at high resolution. III. Simultaneous observations with IRIS and SST. Astrophys. J. 812, 11. DOI. ADS.

Vögler, A., Schüssler, M.: 2007, A solar surface dynamo. Astron. Astrophys. 465, L43. DOI. ADS.

von der Lühe, O., et al.: 2021, The DKIST Visible Tunable Filter (VTF). Solar Phys. 296 (in preparation).

Waidele, M., Roth, M.: 2020, Investigation of surface effects of simple flux tubes using numerical simulations. Astrophys. J. 889, 83. DOI. ADS.

Wang, H., Liu, C.: 2015, Structure and evolution of magnetic fields associated with solar eruptions. Res. Astron. Astrophys. 15, 145. DOI. ADS.

Wang, H., Liu, C., Ahn, K., Xu, Y., Jing, J., Deng, N., Huang, N., Liu, R., Kusano, K., Fleishman, G.D., Gary, D.E., Cao, W.: 2017a, High-resolution observations of flare precursors in the low solar atmosphere. Nat. Astron. 1, 0085. DOI. ADS.

Wang, J., Simões, P.J.A., Jeffrey, N.L.S., Fletcher, L., Wright, P.J., Hannah, I.G.: 2017b, Observations of reconnection flows in a flare on the solar disk. Astrophys. J. Lett. 847, L1. DOI. ADS.

Wang, T.: 2011, Standing slow-mode waves in hot coronal loops: observations, modeling, and coronal seismology. Space Sci. Rev. 158, 397. DOI. ADS.

Wang, T., Sui, L., Qiu, J.: 2007, Direct observation of high-speed plasma outflows produced by magnetic reconnection in solar impulsive events. Astrophys. J. Lett. 661, L207. DOI. ADS.

Wang, Y.-M.: 2016, The ubiquitous presence of looplike fine structure inside solar active regions. Astrophys. J. Lett. 820, L13. DOI. ADS.

Wang, Y.-M., Sheeley, N.R. Jr.: 2003, The solar wind and its magnetic sources at sunspot maximum. Astrophys. J. 587, 818. DOI. ADS.

Wang, Y.-M., Ugarte-Urra, I., Reep, J.W.: 2019, Further evidence for looplike fine structure inside "unipolar" active region plages. Astrophys. J. 885, 34. DOI. ADS.

Wang, Y.-M., Warren, H.P., Muglach, K.: 2016, Converging supergranular flows and the formation of coronal plumes. Astrophys. J. 818, 203. DOI. ADS.

Warren, H.P., Brooks, D.H., Ugarte-Urra, I., Reep, J.W., Crump, N.A., Doschek, G.A.: 2018, Spectroscopic observations of current sheet formation and evolution. Astrophys. J. 854, 122. DOI. ADS.

Watanabe, H., Vissers, G., Kitai, R., Rouppe van der Voort, L., Rutten, R.J.: 2011, Ellerman bombs at high resolution. I. Morphological evidence for photospheric reconnection. Astrophys. J. 736, 71. DOI. ADS.

Watko, J.A., Klimchuk, J.A.: 2000, Width variations along coronal loops observed by TRACE. Solar Phys. 193, 77. DOI. ADS.

Webb, A.R., Roberts, B.: 1978, Vertical motions in an intense magnetic flux tube. II: convective instability. Solar Phys. 59, 249. DOI. ADS.

Webb, D.F., Howard, T.A.: 2012, Coronal mass ejections: observations. Liv. Rev. Solar Phys. 9, 3. DOI. ADS.

Wedemeyer, S., Scullion, E., Steiner, O., de la Cruz Rodriguez, J., Rouppe van der Voort, L.H.M.: 2013, Magnetic tornadoes and chromospheric swirls - definition and classification. In: Cally, P., Erdélyi, R., Norton, A. (eds.) Eclipse on the Coral Sea: Cycle 24 Ascending (GONG 2012, LWS/SDO-5, and SOHO 27), J. Phys. CS-440. DOI. ADS.

Wedemeyer-Böhm, S., Rouppe van der Voort, L.: 2009, Small-scale swirl events in the quiet Sun chromosphere. Astron. Astrophys. 507, L9. DOI. ADS. 
Wedemeyer-Böhm, S., Lagg, A., Nordlund, Å.: 2009, Coupling from the photosphere to the chromosphere and the corona. Space Sci. Rev. 144, 317. DOI. ADS.

Wedemeyer-Böhm, S., Scullion, E., Steiner, O., Rouppe van der Voort, L., de La Cruz Rodriguez, J., Fedun, V., Erdélyi, R.: 2012, Magnetic tornadoes as energy channels into the solar corona. Nature 486, 505. DOI. ADS.

Wehrli, C., Schmutz, W., Shapiro, A.I.: 2013, Correlation of spectral solar irradiance with solar activity as measured by VIRGO. Astron. Astrophys. 556, L3. DOI. ADS.

Welsch, B.T., Longcope, D.W.: 2003, Magnetic helicity injection by horizontal flows in the quiet Sun. I. Mutual-helicity flux. Astrophys. J. 588, 620. DOI. ADS.

Wiegelmann, T., Thalmann, J.K., Schrijver, C.J., De Rosa, M.L., Metcalf, T.R.: 2008, Can we improve the preprocessing of photospheric vector magnetograms by the inclusion of chromospheric observations? Solar Phys. 247, 249. DOI. ADS.

Willis, G.E., Deardorff, J.W.: 1976, A laboratory model of diffusion into the convective planetary boundary layer. Q. J. Roy. Meteorol. Soc. 102, 427. DOI. ADS.

Withbroe, G.L., Noyes, R.W.: 1977, Mass and energy flow in the solar chromosphere and corona. Annu. Rev. Astron. Astrophys. 15, 363. DOI. ADS.

Wöger, F., et al.: 2021, The DKIST Visible Broadband Imager (VBI). Solar Phys. 296 (in preparation).

Woltjer, L.: 1958, On hydromagnetic equilibrium. Proc. Natl. Acad. Sci. USA 44, 833. DOI. ADS.

Wooden, D.H., Woodward, C.E., Harker, D.E., Kelley, M.S., Sitko, M., Reach, W.T., De Pater, I., Gehrz, R.D., Kolokolova, L., Cochran, A.L., McKay, A.J., Reardon, K., Cauzzi, G., Tozzi, G., Christian, D.J., Jess, D.B., Mathioudakis, M., Lisse, C.M., Morgenthaler, J.P., Knight, M.M.: 2013, Comet C/2012 S1 (ISON): observations of the dust grains from SOFIA and of the atomic gas from NSO Dunn and McMath-Pierce solar telescopes (invited). In: AGU Fall Meet. Abs. 2013. ADS.

Woods, D.T., Holzer, T.E., MacGregor, K.B.: 1990a, Lower solar chromosphere-corona transition region. I. Theoretical models with small temperature gradients. Astrophys. J. 355, 295. DOI. ADS.

Woods, D.T., Holzer, T.E., MacGregor, K.B.: 1990b, Lower solar chromosphere-corona transition region. II. Wave pressure effects for a specific form of the heating function. Astrophys. J. Suppl. Ser. 73, 489. DOI. ADS.

Woods, M.M., Harra, L.K., Matthews, S.A., Mackay, D.H., Dacie, S., Long, D.M.: 2017, Observations and modelling of the pre-flare period of the 29 March 2014 X1 flare. Solar Phys. 292, 38. DOI. ADS.

Woods, T.N., Eparvier, F.G., Fontenla, J., Harder, J., Kopp, G., McClintock, W.E., Rottman, G., Smiley, B., Snow, M.: 2004, Solar irradiance variability during the October 2003 solar storm period. Geophys. Res. Lett. 31, L10802. DOI. ADS.

Wright, N.J., Drake, J.J.: 2016, Solar-type dynamo behaviour in fully convective stars without a tachocline. Nature 535, 526. DOI. ADS.

Xu, Y., Cao, W., Liu, C., Yang, G., Jing, J., Denker, C., Emslie, A.G., Wang, H.: 2006, High-resolution observations of multiwavelength emissions during two X-class white-light flares. Astrophys. J. 641, 1210. DOI. ADS.

Xu, Y., Cao, W., Jing, J., Wang, H.: 2010, High resolution observations of white-light emissions from the opacity minimum during an X-class flare. Astron. Nachr. 331, 596. DOI. ADS.

Xu, Y., Cao, W., Jing, J., Wang, H.: 2012, Characteristic size of flare kernels in the visible and near-infrared continua. Astrophys. J. Lett. 750, L7. DOI. ADS.

Xue, Z., Yan, X., Cheng, X., Yang, L., Su, Y., Kliem, B., Zhang, J., Liu, Z., Bi, Y., Xiang, Y., Yang, K., Zhao, L.: 2016, Observing the release of twist by magnetic reconnection in a solar filament eruption. Nat. Commun. 7, 11837. DOI. ADS.

Yamada, M., Yoo, J., Myers, C.E.: 2016, Understanding the dynamics and energetics of magnetic reconnection in a laboratory plasma: review of recent progress on selected fronts. Phys. Plasmas 23, 055402. DOI. ADS.

Yamamoto, T.T., Kusano, K.: 2012, Preprocessing magnetic fields with chromospheric longitudinal fields. Astrophys. J. 752, 126. DOI. ADS.

Yan, Y., Deng, Y., Karlický, M., Fu, Q., Wang, S., Liu, Y.: 2001, The Magnetic Rope Structure and Associated Energetic Processes in the 2000 July 14 Solar Flare. Astrophys. J. Lett. 551(1), L115. DOI. ADS.

Yeates, A.R., Hornig, G.: 2016, The global distribution of magnetic helicity in the solar corona. Astron. Astrophys. 594, A98. DOI. ADS.

Yelles Chaouche, L., Kuckein, C., Martínez Pillet, V., Moreno-Insertis, F.: 2012, The three-dimensional structure of an active region filament as extrapolated from photospheric and chromospheric observations. Astrophys. J. 748, 23. DOI. ADS.

Yeo, K.L., Solanki, S.K., Norris, C.M., Beeck, B., Unruh, Y.C., Krivova, N.A.: 2017, Solar irradiance variability is caused by the magnetic activity on the solar surface. Phys. Rev. Lett. 119, 091102. DOI. ADS.

Yokoyama, T., Shimojo, M., Okamoto, T.J., Iijima, H.: 2018, ALMA observations of the solar chromosphere on the polar limb. Astrophys. J. 863, 96. DOI. ADS. 
Young, P.R., Tian, H., Peter, H., Rutten, R.J., Nelson, C.J., Huang, Z., Schmieder, B., Vissers, G.J.M., Toriumi, S., Rouppe van der Voort, L.H.M., Madjarska, M.S., Danilovic, S., Berlicki, A., Chitta, L.P., Cheung, M.C.M., Madsen, C., Reardon, K.P., Katsukawa, Y., Heinzel, P.: 2018, Solar ultraviolet bursts. Space Sci. Rev. 214, 120. DOI. ADS.

Yurchyshyn, V., Abramenko, V., Kosovichev, A., Goode, P.: 2014, High resolution observations of chromospheric jets in sunspot umbra. Astrophys. J. 787, 58. DOI. ADS.

Yurchyshyn, V., Kumar, P., Abramenko, V., Xu, Y., Goode, P.R., Cho, K.-S., Lim, E.-K.: 2017, Highresolution observations of a white-light flare with NST. Astrophys. J. 838, 32. DOI. ADS.

Zachariadis, T.G., Alissandrakis, C.E., Banos, G.: 1987, Observations of Ellerman bombs in H $\alpha$. Solar Phys. 108, 227. DOI. ADS.

Zeuner, F., Feller, A., Iglesias, F.A., Solanki, S.K.: 2018, Detection of spatially structured scattering polarization of Sr I 4607.3 A with the Fast Solar Polarimeter. Astron. Astrophys. 619, A179. DOI. ADS.

Zhang, M., Low, B.C.: 2001, Magnetic flux emergence into the solar corona. I. Its role for the reversal of global coronal magnetic fields. Astrophys. J. 561, 406. DOI. ADS.

Zhang, M., Low, B.C.: 2005, The hydromagnetic nature of solar coronal mass ejections. Annu. Rev. Astron. Astrophys. 43, 103. DOI. ADS.

Zhang, M., Flyer, N., Low, B.C.: 2006, Magnetic field confinement in the corona: the role of magnetic helicity accumulation. Astrophys. J. 644, 575. DOI. ADS.

Zhang, Y.Z., Shibata, K., Wang, J.X., Mao, X.J., Matsumoto, T., Liu, Y., Su, J.T.: 2012, Revision of solar spicule classification. Astrophys. J. 750, 16. DOI. ADS.

Zhao, X., Hoeksema, J.T.: 1995, Prediction of the interplanetary magnetic field strength. J. Geophys. Res. 100, 19. DOI. ADS.

Zharkova, V.V., Zharkov, S.I.: 2007, On the origin of three seismic sources in the proton-rich flare of 2003 October 28. Astrophys. J. 664, 573. DOI. ADS.

Zhou, Y.: 2017, Rayleigh-Taylor and Richtmyer-Meshkov instability induced flow, turbulence, and mixing. II. Phys. Rep. 723, 1. DOI. ADS.

Zhu, X., Wang, H., Du, Z., He, H.: 2016, Forced field extrapolation of the magnetic structure of the H $\alpha$ fibrils in the solar chromosphere. Astrophys. J. 826, 51. DOI. ADS.

Zhugzhda, Y.D., Bromm, V., Ulmschneider, P.: 1995, Propagation of nonlinear longitudinal-transverse waves along magnetic flux tubes in the solar atmosphere. II. The treatment of shocks. Astron. Astrophys. 300, 302. ADS.

Zirker, J.B.: 1993, Coronal heating. Solar Phys. 148, 43. DOI. ADS.

Zirker, J.B., Engvold, O., Martin, S.F.: 1998, Counter-streaming gas flows in solar prominences as evidence for vertical magnetic fields. Nature 396, 440. DOI. ADS.

Zuccher, S., Ricca, R.L.: 2017, Relaxation of twist helicity in the cascade process of linked quantum vortices. Phys. Rev. E 95, 053109. DOI. ADS.

Zweibel, E.G.: 1989, Magnetic reconnection in partially ionized gases. Astrophys. J. 340, 550. DOI. ADS.

Zweibel, E.G.: 2015, Ambipolar diffusion. In: Lazarian, A., de Gouveia Dal Pino, E.M., Melioli, C. (eds.) Magnetic Fields in Diffuse Media Astrophys. Space Sci. Lib. 407, 285. DOI. ADS.

Zweibel, E.G., Lawrence, E., Yoo, J., Ji, H., Yamada, M., Malyshkin, L.M.: 2011, Magnetic reconnection in partially ionized plasmas. Phys. Plasmas 18, 111211. DOI. ADS. 